\title{
Classification of Bicovariant Differential Calculi on Quantum Groups of Type A, B, C and D
}

\author{
Konrad Schmüdgen, Axel Schüler
}

Fachbereich Mathematik/Informatik, Universität Leipzig, Augustusplatz 10, D-04109 Leipzig, Germany; E-mail: schmuedgen@mathematik.uni-leipzig.d400.de

Received: 5 January 1994

\begin{abstract}
Under the assumptions that $q$ is not a root of unity and that the differentials $d u_{j}^{i}$ of the matrix entries span the left module of first order forms, we classify bicovariant differential calculi on quantum groups $A_{n-1}, B_{n}, C_{n}$ and $D_{n}$. We prove that apart one dimensional differential calculi and from finitely many values of $q$, there are precisely $2 n$ such calculi on the quantum group $A_{n-1}=S L_{q}(n)$ for $n \geqq 3$. All these calculi have the dimension $n^{2}$. For the quantum groups $B_{n}, C_{n}$ and $D_{n}$ we show that except for finitely many $q$ there exist precisely two $N^{2}$-dimensional bicovariant calculi for $N \geqq 3$, where $N=2 n+1$ for $B_{n}$ and $N=2 n$ for $C_{n}, D_{n}$. The structure of these calculi is explicitly described and the corresponding ad-invariant right ideals of ker $\varepsilon$ are determined. In the limit $q \rightarrow 1$ two of the $2 n$ calculi for $A_{n-1}$ and one of the two calculi for $B_{n}, C_{n}$ and $D_{n}$ contain the ordinary classical differential calculus on the corresponding Lie group as a quotient.
\end{abstract}

\section{Introduction}

Non-commutative differential calculus is a basic tool for further applications of quantum groups and for studying non-commutative geometry on quantum spaces. A general framework for bicovariant differential calculi on quantum groups (Hopf algebras) is developed by S.L. Woronowicz [Wo2]. Following general ideas of A. Connes [C], differential forms are the basic objects of this theory. Examples of covariant differential calculi are constructed and studied (for instance) in [Wo1, Wo2, WZ, R2, CSWW, J, SWZ, Su and BM]. In general there are many nonisomorphic bicovariant differential calculi on a given quantum group, and no functorial method is known to construct a "natural" differential calculus as in classical differential geometry on Lie groups. The aim of this paper is to classify all bicovariant calculi under "reasonable" assumptions and to select one or a few distinguished calculi in this way. Despite the rather extensive literature about differential calculi on quantum groups, the classification problem has been treated only in the special case $N=2$, cf. [St, MH].

The aim of this paper is to classify (under certain assumptions) all bicovariant differential calculi on the quantum groups corresponding to the four series of 
classical simple Lie groups $A_{n-1}, B_{n}, C_{n}$ and $D_{n}$ as defined in [FRT]. Let $u=\left(u_{j}^{i}\right)_{i, j=1, \ldots, N}$ denote the corresponding fundamental matrix. The crucial assumptions for our classification are that the deformation parameter $q$ is not a root of unity and that the differentials $d u_{j}^{i}, i, j=1, \ldots, N$, generate the left module of first order forms. Further we assume throughout that $N \geqq 3$. We briefly discuss the main results of this paper. The first main result states that except for finitely many values of $q$ (see Remark 2 in Sect. 2) there exist precisely $2 N$ bicovariant calculi of dimension greater than one on $S L_{q}(N)$. They are parametrized by one of the signs,+- (which correspond to the numbers $q$ and $q^{-1}$ ) and by an $N$-th root $q_{k}$ of $q^{2}$. The corresponding calculi are denoted by $(\Gamma( \pm, k), d)$. Note that the quantum group $S L_{q}(2)$ has, in contrast to the preceding, only two such bicovariant calculi. The second main result is that apart from finitely many $q$ (see Remark 2 in Sect. 6) there are precisely two $N^{2}$-dimensional bicovariant calculi on $O_{q}(N)$ and $S p_{q}(N)$. They are denoted by $\left(\Gamma_{ \pm}, d\right)$. It turns out that all calculi occurring in our classification are inner (i.e. $d a=\eta a-a \eta$ for some right- and left-invariant form $\eta$ ) and that all of them can be constructed by a method of B. Jurčo [J]. Bicovariant differential calculi on $S U_{q}(N)$ and on $O_{q}(N)$ have been already introduced and studied in [CSWW]. The calculi therein correspond to our $(\Gamma(+, k), d)$ for $S U_{q}(N)$ (the $N^{\text {th }}$ root of $q^{2}$ is implicit in the choice of the $R$-matrix in [CSWW]) and to our $\left(\Gamma_{+}, d\right)$ for $O_{q}(N)$. Further, each calculus in our list admits a limit as $q \rightarrow 1$ (in an appropriate sense), which is bicovariant differential calculus on the corresponding classical Lie group. For the two calculi $(\Gamma(+, k), d)$ and $(\Gamma(-, k), d)$ on $S L_{q}(N)$ with $q_{k}$ belonging to the first branch of $N^{\text {th }}$ root, and for the calculus $\left(\Gamma_{+}, d\right)$ on $O_{q}(N)$ and $S p_{q}(N)$, these limits contain the usual standard differential calculi on the Lie groups as a quotient, cf. Sects. 5 and 9. That is, if we require the latter behaviour of the classical limits $q \rightarrow 1$, we have two distinguished bicovariant differential calculi on $S L_{q}(N)$ and one distinguished bicovariant calculus on $O_{q}(N)$ and $S p_{q}(N)$. This outcome of our classification seems to be very promising for further applications of these calculi and for their use in the study of non-commutative geometry on quantum groups.

Our main results on classification of bicovariant differential calculi (Theorems 2.2 and 6.2) are obtained by means of a classification of the associated ad-invariant right ideals of ker $\varepsilon$ (Theorems 2.1 and 6.1). For this the explicit decompositions of parts of the adjoint representations into irreducible components are needed. Here the assumption that $q$ is not a root of unity is essential and is used in order to have similar results as the classical case. The proofs of Theorems 2.1 and 6.1 require a number of rather long computations. In order to limit the size of the paper, not all computations are carried out. Sometimes a sample is given or only the result of a computation is stated. But in these cases all necessary facts are mentioned.

This paper is organized as follows. In Sect. 2 we collect some basic definitions and preliminary facts needed later. The classification of bicovariant differential calculi and their ad-invariant right ideals is carried out in Part I (Sects. 2-5) for the quantum group $S L_{q}(N)$ and in Part II (Sects. 6-9) for the quantum groups $O_{q}(N)$ and $S p_{q}(N)$. In both cases the main results are two theorems stated in Sect. 2, resp. 6. Their proofs are given in Sects. 4 and 8 . These proofs essentially depend on some properties of certain interwiners which are built from the matrix $\hat{R}$ and their spectral projections. The necessary technical tools are provided in Sects. 3 and 7. The limits of our calculi for $q \rightarrow 1$ are investigated in Sects. 5 and 9 . 
The main result of this paper for $B_{n}, C_{n}, D_{n}$ (Theorem 6.2) is announced in [SS], see also Remark 1 in Sect. 6 for a correction.

\section{Preliminaries}

In this paper $\mathscr{A}$ denotes one of the Hopf algebras for the quantum groups of type $A, B, C$ or $D$ as defined in [FRT], Subsects. 1.3 and 1.4. Throughout we assume that $N \geqq \mathbf{3}$ and with the exception of Sects. 5 and 9 that the (non-zero) deformation parameter $\boldsymbol{q}$ is not a root of unity. (A closer look at the proofs shows that it suffices to assume that $q^{n} \neq 1$ for all $n \in \mathbb{N}, n \leqq c(N)$, where $c(N)$ is some constant depending only on $N$. Of course, some considerations are also valid without restrictions on $q$.)

Unless it is explicitly stated otherwise, we use the Einstein convention to sum over repeated indices. However, sometimes we write the sum if ambiguities are possible. In any case, the meaning will be clear from the context.

We denote by $\Delta$ the comultiplication, $\varepsilon$ the counit, $\kappa$ the antipode and $\mathbb{1}$ the unit element of $\mathscr{A}$. By definition [FRT], the algebra $\mathscr{A}$ is generated by $\mathbb{1}$ and the $N^{2}$ entries $u_{j}^{i}, i, j=1, \ldots, N$, of the fundamental matrix $u=\left(u_{j}^{i}\right)$. For $k \in \mathbb{N}$, let $\mathscr{A}_{k}$ be the linear span of products $u_{j_{1}}^{i_{1}} \ldots u_{j_{k}}^{i_{k}}$. We set $\tilde{x}:=x-\varepsilon(x) \mathbb{1}$ for $x \in \mathscr{A}$ and $\tilde{\mathscr{B}}:=\{\tilde{x}: x \in \mathscr{B}\}$ for a subset $\mathscr{B}$ of $\mathscr{A}$. The linear span of a set $\left\{a_{i}: i \in I\right\}$ is denoted by $\left\langle a_{i}: i \in I\right\rangle$ and the flip operator of the tensor product by $P$, i.e. $P_{n m}^{i j}=\delta_{i m} \delta_{j n}$. If $A$ is a linear mapping or a matrix, $A^{t}$ denotes the transpose of $A$ and lower indices of $A$ always refer to the components of a tensor product where $A$ acts. Let $\theta$ denote the Heaviside symbol, i.e. $\theta(m)=1$ if $m>0$ and $\theta(m)=0$ if $m \leqq 0$.

By a representation of $\mathscr{A}$ we mean a representation of the coalgebra $\mathscr{A}$, i.e. a right comodule for the coalgebra $\mathscr{A}$. Often we identify representations and the corresponding matrices by fixing some basis of the underlying vector space. Throughout, $u$ denotes the fundamental representation of $\mathscr{A}$. As usual, $v^{c}$ is the contragredient representation of $v$ and $\operatorname{Mor}(v, w)$ is the space of intertwiners of representations $v$ and $w$. We write $\operatorname{Mor}(v)$ for $\operatorname{Mor}(v, v)$. Since we assume that $q$ is not a root of unity, the representation theory of the quantum group $\mathscr{A}$ is completely similar to the classical case ([L, R2]; cf. also $[\mathrm{PW}]$, chapter 8). All finite dimensional representations of $\mathscr{A}$ are $q$-deformations of representations of the corresponding classical matrix group, so they can be labelled by Young tableaus similar to the classical case (see, for instance, [BR or H]). We freely use these facts in the proofs of Theorems 2.1 and 6.1. Concerning the classical representation theory, we follow the standard notation, cf. [H]. The trivial one dimensional representation is denoted by [0]. The following simple lemma is needed in the sequel.

Lemma 1.1. If $\mathscr{R}$ is a right ideal of $\operatorname{ker} \varepsilon$ such that $\operatorname{ker} \varepsilon=\mathscr{R}+\tilde{\mathscr{A}}_{1}$ and if $\mathscr{R}_{12}:=$ $\mathscr{R} \cap\left(\mathscr{A}_{0}+\mathscr{A}_{1}+\mathscr{A}_{2}\right)$, then we have $\mathscr{R}=\mathscr{R}_{12} \cdot \mathscr{A}$.

Proof. Let $\mathscr{A}^{k}:=\mathscr{A}_{0}+\cdots+\mathscr{A}_{k}, k \in \mathbb{N}$. Obviously, $\mathscr{R}_{12} \cdot \mathscr{A} \subseteq \mathscr{R}$. We prove the converse by induction on $k$. Suppose that $\mathscr{R} \cap \mathscr{A}^{k} \subseteq \mathscr{R}_{12} \cdot \mathscr{A}$. Let $a \in \mathscr{R} \cap \mathscr{A}^{k+1}$. We write $a$ as $a=b_{i}^{j} u_{j}^{i}+b_{0}$ with $b_{i}^{j}, b_{0} \in \mathscr{A}^{k}$. Since $\mathscr{A}=\mathscr{R}+\mathscr{A}^{1}$ by the assumption ker $\varepsilon=\mathscr{R}+\tilde{\mathscr{A}}_{1}$, we have $b_{i}^{j}=a_{i}^{j}+c_{i}^{j}$ and $b_{0}=a_{0}+c_{0}$ with $a_{i}^{j}, a_{0} \in \mathscr{R}$ and $c_{i}^{j}, c_{0} \in \mathscr{A}^{1}$. By induction hypothesis, $a_{1}:=a_{i}^{j} u_{j}^{i}+a_{0}$ is in $\mathscr{R}_{12} \cdot \mathscr{A}$. Since $a-a_{1}=c_{i}^{j} u_{j}^{i}+c_{0} \in \mathscr{R} \cap \mathscr{A}^{2}=\mathscr{R}_{12} \cdot \mathbb{1}$, we conclude that $a \in \mathscr{R}_{12} \cdot \mathscr{A}$. 
Bicovariant Bimodules

Definition 1.1. A bicovariant bimodule over $\mathscr{A}$ is a triple $\left(\Gamma, \Delta_{L}, \Delta_{R}\right)$ of a bimodule $\Gamma$ over $\mathscr{A}$ and of linear mappings $\Delta_{L}: \Gamma \rightarrow \mathscr{A} \otimes \Gamma$ and $\Delta_{R}: \Gamma \rightarrow \Gamma \otimes \mathscr{A}$ such that:

1. $\left(\Gamma, \Delta_{L}\right)$ is a left comodule over $\mathscr{A}$, i.e. $\left(\mathrm{id} \otimes \Delta_{L}\right) \Delta_{L}=(\Delta \otimes \mathrm{id}) \Delta_{L}$ and $(\varepsilon \otimes \mathrm{id}) \Delta_{L}=\mathrm{id}$.

2. $\left(\Gamma, \Delta_{R}\right)$ is a right comodule over $\mathscr{A}$, i.e. $\left(\Delta_{R} \otimes \mathrm{id}\right) \Delta_{R}=\left(\mathrm{id} \otimes \Delta_{)} \Delta_{R}\right.$ and $(\mathrm{id} \otimes \varepsilon) \Delta_{R}=\mathrm{id}$. $\omega \in \Gamma$.

3. $\Delta_{L}(a \omega b)=\Delta(a) \Delta_{L}(\omega) \Delta(b)$ and $\Delta_{R}(a \omega b)=\Delta(a) \Delta_{R}(\omega) \Delta(b)$ for $a, b \in \mathscr{A}$ and

4. $\left(\mathrm{id} \otimes \Delta_{R}\right) \Delta_{L}=\left(\Delta_{L} \otimes \mathrm{id}\right) \Delta_{R}$.

Let $\left(\Gamma, \Delta_{L}, \Delta_{R}\right)$ be a bicovariant bimodule over $\mathscr{A}$. An element $\omega \in \Gamma$ is called left-invariant if $\Delta_{L}(\omega)=\mathbb{1} \otimes \omega$ and right-invariant if $\Delta_{R}(\omega)=\omega \otimes \mathbb{1}$. The vector space of left-invariant elements is denoted by $\Gamma_{\text {inv }}$. The canonical projection $P_{\mathrm{inv}}: \Gamma \rightarrow \Gamma_{\mathrm{inv}}$ is defined by $P_{\mathrm{inv}}(\omega)=\sum_{i} \kappa\left(a_{i}\right) \omega_{i}$ if $\Delta_{L}(\omega)=\sum_{i} a_{i} \otimes \omega_{i}$.

The structure of bicovariant bimodules is completely characterized by Theorems 2.3 and 2.4 in [Wo2]. We recall the corresponding result:

Let $\left(\Gamma, \Delta_{L}, \Delta_{R}\right)$ be a bicovariant bimodule over $\mathscr{A}$ and let $\left(\omega_{i}\right)_{i \in I}$ be a basis of the vector space $\Gamma_{\mathrm{inv}}$. Then there exist matrices $v=\left(v_{j}^{i}\right)_{i, j \in I}$ and $f=\left(f_{j}^{i}\right)_{i, j \in I}$ of elements $v_{j}^{i} \in \mathscr{A}$ and of functionals $f_{j}^{i} \in \mathscr{A}^{\prime}$ such that for $a, b \in \mathscr{A}$ and $i, j \in I$ :

(i) $\omega_{i} a=\left(f_{r}^{i} * a\right) \omega_{r}$ and $\Delta_{R}\left(\omega_{i}\right)=\omega_{r} \otimes v_{i}^{r}$.

(ii) $v=\left(v_{i}^{j}\right)$ is a representation of the coalgebra $\mathscr{A}$, i.e. $\Delta\left(v_{j}^{i}\right)=v_{n}^{i} \otimes v_{j}^{n}$ and $\varepsilon\left(v_{j}^{i}\right)=\delta_{i j}$.

(iii) $f=\left(f_{j}^{i}\right)$ is a representation of the algebra $\mathscr{A}$, i.e. $f_{j}^{i}(a b)=f_{k}^{i}(a) f_{j}^{k}(b)$ and $f_{j}^{i}(\mathbb{1})=\delta_{i j}$.

(iv) $v_{i}^{n}\left(a * f_{j}^{n}\right)=\left(f_{n}^{i} * a\right) v_{n}^{j}$.

The set $\left(\omega_{i}\right)_{i \in I}$ is a free left module basis of $\Gamma$. As usual, we have set $a * f:=(f \otimes \mathrm{id}) \Delta(a)$ and $f * a:=(\mathrm{id} \otimes f) \Delta(a)$.

Conversely, if $\left(\omega_{i}\right)_{i \in I}$ is a basis of a certain vector space $\Gamma_{0}$ and if $v=\left(v_{j}^{i}\right)_{i, j \in I}$ and $f=\left(f_{j}^{i}\right)_{i, j \in I}$ are matrices with $v_{j}^{i} \in \mathscr{A}$ and $f_{j}^{i} \in \mathscr{A}^{\prime}$ satisfying (ii), (iii) and (iv), then there exists a unique bicovariant bimodule $\Gamma$ such that $\Gamma_{0}=\Gamma_{\text {inv }}$ and (i) holds. In the situation just described we simply write $(v, f)$ for the corresponding bicovariant bimodule $\Gamma$.

Lemma 1.2. Let $\Gamma_{1}=(v, f)$ and $\Gamma_{2}=(w, g)$ be bicovariant bimodules and define $f * g:=(f \otimes g) \Delta$. Then the pair $(v \otimes w, f * g)$ is also bicovariant bimodule which will be denoted by $\Gamma_{1} \otimes \Gamma_{2}$.

Proof. Clearly, it suffices to check that $v \otimes w$ and $f * g$ satisfy again the compatibility condition (1.1), i.e. $(v \otimes w)_{i j}^{n m}\left(a *(f * g)_{r s}^{n m}\right)=v_{i}^{n}\left(w_{j}^{m}\left(a * f_{r}^{n}\right) * g_{s}^{m}\right)=v_{i}^{n}\left(g_{m}^{j} *\right.$ $\left.\left.\left(a * f_{r}^{n}\right) w_{m}^{s}\right)=v_{i}^{n}\left(\left(g_{m}^{j} * a\right) * f_{r}^{n}\right) w_{m}^{s}=f_{n}^{i} *\left(g_{m}^{j} * a\right)\right) v_{n}^{r} w_{m}^{s}=\left((f * g)_{n m}^{i j} * a\right)(v \otimes w)_{n m}^{r s}$.

Lemma 1.3. Let $v_{j}^{i} \in \mathscr{A}$ and $f_{j}^{i} \in \mathscr{A}^{\prime}, i, j \in I$, be such that the matrices $v=\left(v_{j}^{i}\right)$ and $f=\left(f_{j}^{i}\right)$ satisfy (ii) and (iii), respectively. Set $T_{i m}^{n j}:=f_{j}^{i}\left(u_{m}^{n}\right)$. Then the compatibility condition (1.1) is fulfilled if and only if $T \equiv\left(T_{i m}^{n j}\right) \in \operatorname{Mor}(v \otimes u, u \otimes v)$. 
Proof. Using (iii) it is easily seen that (1.1) is satisfied for $a \cdot b$ provided that it is valid for $a$ and for $b$. Since $f_{j}^{i}(\mathbb{1})=\delta_{i j},(1.1)$ is true for $a=\mathbb{1}$. Thus it is sufficient to check (1.1) for the elements $u_{s}^{r}$. From

$$
v_{i}^{n}\left(u_{s}^{r} * f_{j}^{n}\right)=T_{n m}^{r j} v_{i}^{n} u_{s}^{m} \quad \text { and }\left(f_{n}^{i} * u_{s}^{r}\right) v_{n}^{j}=u_{m}^{r} v_{n}^{j} T_{i s}^{m n},
$$

we conclude that (1.1) is equivalent to $T \in \operatorname{Mor}(v \otimes u, u \otimes v)$.

\section{Bicovariant Differential Calculi}

Definition 1.2. A first order differential calculus (or briefly, a differential calculus) over $\mathscr{A}$ is a pair $(\Gamma, d)$ of a bimodule $\Gamma$ over $\mathscr{A}$ and a linear mapping $d: \mathscr{A} \rightarrow \Gamma$ such that $d(a b)=d a \cdot b+a \cdot d b$ for $a, b \in \mathscr{A}$ and $\Gamma=\langle a \cdot d b: a, b \in \mathscr{A}\rangle$.

Definition 1.3. A first order differential calculus $(\Gamma, d)$ over $\mathscr{A}$ is called bicovariant if there exist mappings $\Delta_{L}: \Gamma \rightarrow \mathscr{A} \otimes \Gamma$ and $\Delta_{R}: \Gamma \rightarrow \Gamma \otimes \mathscr{A}$ such that:

1. $\left(\Gamma, \Delta_{L}, \Delta_{R}\right)$ is a bicovariant bimodule.

2. $\Delta_{L}(d a)=(\mathrm{id} \otimes d) \Delta(a)$ and $\Delta_{R}(d a)=(d \otimes \mathrm{id}) \Delta(a)$ for $a \in \mathscr{A}$.

Note that the mappings $\Delta_{L}$ and $\Delta_{R}$ (if they exist) are uniquely determined by $\Gamma$ and $d$. By Propositions 1.2-1.4 in [Wo2], the preceding definition of bicovariance is equivalent to the one given in [Wo2].

A bicovariant differential calculus $(\Gamma, d)$ is called inner if there exists an element $\omega \in \Gamma$ which is left- and right-invariant such that $d a=\omega a-a \omega$ for all $a \in \mathscr{A}$. We shall say that two differential calculi $\left(\Gamma_{1}, d_{1}\right)$ and $\left(\Gamma_{2}, d_{2}\right)$ over $\mathscr{A}$ are isomorphic if there is a bimodule isomorphism $\Psi$ of $\Gamma_{1}$ onto $\Gamma_{2}$ such that $\Psi \circ d_{1}=d_{2}$.

\section{Adjoint Representation}

For $a \in \mathscr{A}$, we set $\operatorname{ad}(a):=\sum_{i} b_{i} \otimes \kappa\left(a_{i}\right) c_{i}$, where the elements $a_{i}, b_{i}, c_{i} \in \mathscr{A}$ are defined by $(\mathrm{id} \otimes \Delta) \Delta(a)=\sum_{i} a_{i} \otimes b_{i} \otimes c_{i}$. The map ad: $\mathscr{A} \rightarrow \mathscr{A} \otimes \mathscr{A}$ is a representation of the coalgebra $\mathscr{A}$ called the adjoint representation of $\mathscr{A}$. In particular, we have

$$
\operatorname{ad}\left(u_{j_{1}}^{i_{1}} \ldots u_{j_{k}}^{i_{k}}\right)=u_{m_{1}}^{n_{1}} \ldots u_{m_{k}}^{n_{k}} \otimes\left(u^{c}\right)_{i_{k}}^{n_{k}} \ldots\left(u^{c}\right)_{i_{1}}^{n_{1}} u_{j_{1}}^{m_{1}} \ldots u_{j_{k}}^{m_{k}}
$$

for $i_{1}, \ldots, i_{k} \in\{1, \ldots, N\}$ and $k \in \mathbb{N}$. Let $\mathbf{I}_{k}$ denote the linear mapping of $\left(\mathbb{C}^{N}\right)^{\otimes 2 k}$ into $\mathscr{A}_{k}$ defined by $\mathbf{I}_{k}\left(e_{n_{k} \ldots n_{1} m_{1} \ldots m_{k}}\right):=u_{m_{1}}^{n_{1}} \ldots u_{m_{k}}^{n_{k}}$, where $\left\{e_{r_{1} \ldots r_{2 k}}\right\}$ is the standard basis of $\left(\mathbb{C}^{N}\right)^{\otimes 2 k}$. By (1.2), $\mathbf{I}_{k} \in \operatorname{Mor}\left(\left(u^{c}\right)^{\otimes k}, \operatorname{ad}\left\lceil\mathscr{A}_{k}\right)\right.$.

Lemma 1.4. (i) If $\mathscr{V}$ is an ad-invariant linear subspace of $\mathscr{A}$, then $\tilde{\mathscr{V}}$ is also ad-invariant and the map $a \rightarrow \tilde{a}$ belongs to $\operatorname{Mor}(\operatorname{ad} \Gamma \mathscr{V}, \operatorname{ad}\lceil\tilde{V})$.

(ii) For $k \in \mathbb{N}, \operatorname{ad}\left\lceil\mathscr{A}_{k} \cong \operatorname{ad}\left\lceil\tilde{\mathscr{A}}_{k}\right.\right.$ if and only if $k \notin n \cdot \mathbb{N}$ for $A_{n-1}$ resp. $k \notin 2 \mathbb{N}$ for $B_{n}, C_{n}, D_{n}$.

Proof. (i) follows easily from the Hopf algebra axioms for the counit and the antipode. Obviously, the map $a \rightarrow \tilde{a}$ of $\mathscr{A}_{k}$ onto $\tilde{\mathscr{A}}_{k}$ is injective if and only if $\mathbb{1} \notin \mathscr{A}_{k}$. The latter is equivalent to $k \notin n \cdot \mathbb{N}$ for the quantum group $A_{n-1}$ and to $k \notin 2 \cdot \mathbb{N}$ for the quantum groups $B_{n}, C_{n}, D_{n}$. This yields (ii).

In particular, ker $\varepsilon$ is ad-invariant. According to Theorems 1.5 and 1.8 in [Wo2], there is a one-to-one correspondence between bicovariant differential calculi $(\Gamma, d)$ over $\mathscr{A}$ and ad-invariant right ideals $\mathscr{R}$ of ker $\varepsilon$ given by $\mathscr{R}=\left\{a \in \operatorname{ker} \varepsilon: P_{\text {inv }}(d a)=\right.$ $0\}$, see formula (5.14) in [Wo2]. Further, since $\Gamma_{\text {inv }}$ and $(\operatorname{ker} \varepsilon / \mathscr{R})^{\prime}$ form a dual pair of vector spaces $\left([\mathrm{Wo} 2]\right.$, p. 161) $\operatorname{dim} \Gamma_{\mathrm{inv}}=\operatorname{codim} \mathscr{R}$. We call the latter the dimension of the bicovariant differential calculus $(\Gamma, d)$. 
Lemma 1.5. Let $\mathscr{R}$ be the ad-invariant right ideal of $\mathrm{ker} \varepsilon$ associated with the bicovariant calculus $(\Gamma, d)$ over $\mathscr{A}$. The following statements are equivalent:

(i) $\Gamma=\left\langle a \cdot d u_{j}^{i}: a \in \mathscr{A}, i, j=1, \ldots, N\right\rangle$,

(ii) $\Gamma_{\mathrm{inv}}=\left\langle P_{\mathrm{inv}}\left(d u_{j}^{i}\right): i, j=1, \ldots, N\right\rangle$,

(iii) $\operatorname{ker} \varepsilon=\mathscr{R}+\tilde{\mathscr{A}}_{1}$.

Proof. By definition, we have $\omega_{i j}:=P_{\text {inv }}\left(d u_{j}^{i}\right)=\kappa\left(u_{k}^{i}\right) d u_{j}^{k}$. (i) $\rightarrow$ (ii) is trivial, since $P_{\text {inv }}\left(a d u_{j}^{i}\right)=\varepsilon(a) \omega_{i j}$. (ii) $\rightarrow$ (i) follows at once from Theorem 2.1.1 in [Wo2]. (ii) $\rightarrow$ (iii): If $a \in \operatorname{ker} \varepsilon$ and $P_{\text {inv }}(d a)=\alpha_{i j} \omega_{i j}$ with $\alpha_{i j} \in \mathbb{C}$, then $a-\alpha_{i j} \tilde{u}_{j}^{i} \in \mathscr{R}$. (iii) $\rightarrow$ (ii): Let $\omega=P_{\text {inv }}\left(a_{i} d b_{i}\right) \in \Gamma_{\text {inv }}$. Then $a:=\varepsilon\left(a_{i}\right) \tilde{b}_{i} \in \operatorname{ker} \varepsilon$ and $\omega=P_{\text {inv }}(d a)$. By (iii), $a=r+\alpha_{i j} \tilde{u}_{j}^{i}$ for some $r \in \mathscr{R}$ and $\alpha_{i j} \in \mathbb{C}$. Hence $\omega=\alpha_{i j} \omega_{i j}$.

\section{Part I: Classification of Bicovariant Differential Calculi on $S L_{q}(N)$}

\section{Definitions and Main Results}

In this section we state our main results, and collect some notations and facts which will be used throughout the following four sections. Let $\mathscr{A}$ denote the Hopf algebra of the quantum group $S L_{q}(N)$ as defined in [FRT], Definition 3. We set $Q:=q-q^{-1}$ and $Q_{+}:=q+q^{-1}$. The corresponding matrix $\hat{R}$ for $S L_{q}(N)$ is given by

$$
\hat{R}_{j m}^{i n}:=\delta_{i m} \delta_{j n}\left(1+(q-1) \delta_{i n}\right)+Q \delta_{i j} \delta_{n m} \theta(n-i)
$$

for $i, j, n, m=1, \ldots, N$. The matrix $\hat{R}$ can be written as $\hat{R}=q P_{+}-q^{-1} P_{-}$, where

$$
P_{+}:=Q_{+}^{-1}\left(\hat{R}+q^{-1} I\right) \text { and } P_{-}:=Q_{+}^{-1}(-\hat{R}+q I)
$$

are projections. We have (cf. [FRT], Theorem 4)

$$
q^{-2 i} \kappa\left(u_{n}^{i}\right) u_{i}^{m}=q^{-2 n} \delta_{n m} \quad \text { and } q^{2 i} u_{n}^{i} \kappa\left(u_{i}^{m}\right)=q^{2 n} \delta_{n m} .
$$

The Ad-Invariant Right Ideals $\mathscr{R}( \pm, k)$

We shall use the abbreviations

$$
\begin{aligned}
\mathfrak{s} & :=\sum_{i=1}^{N} q^{-2 i}, \mathfrak{s}_{+}:=1+\mathfrak{s}+q^{-2 N-2}, \mathfrak{s}_{-}:=\mathfrak{s}-q^{-2}-q^{-2 N}, \\
\mathfrak{t}_{+} & :=1+\mathfrak{s}, \mathfrak{t}_{-}:=\mathfrak{s}-q^{-2 N}, \mathfrak{s}_{+}^{\prime}:=\mathfrak{s}-q^{-2}+1, \mathfrak{s}_{-}^{\prime}:=\mathfrak{s}-q^{-2 N}+q^{-2 N-2}
\end{aligned}
$$

and

$$
\left(v_{ \pm}\right)_{j}^{i}:=\left(P_{ \pm}\right)_{n m}^{k i} q^{-2 k} u_{k}^{n} u_{j}^{m}, \quad i, j=1, \ldots, N .
$$

We denote by $U$ and $V_{ \pm}$the $q$-traces of the matrices $\left(u_{j}^{i}\right)$ and $\left(\left(v_{ \pm}\right)_{j}^{i}\right)$, respectively, i.e. we set

$$
U:=\sum_{i} q^{-2 i} u_{i}^{i} \quad \text { and } \quad V_{ \pm}:=\sum_{i} q^{-2 i}\left(v_{ \pm}\right)_{i}^{i}
$$

If $\mathscr{F}$ is an $N^{2}$-dimensional vector space with some distinguished basis, we define a projection $P_{0}: \mathscr{F} \rightarrow \mathscr{F}$ by the matrix $\left(P_{0}\right)_{j m}^{i n}:=\mathfrak{s}^{-1} q^{-2 i} \delta_{i n} \delta_{j m}$. Further, we define linear mappings $S_{ \pm}: \mathscr{A}_{2} \rightarrow \mathscr{A}_{2}$ by

$$
S_{ \pm}\left(u_{j}^{i} u_{m}^{n}\right):=\left(P_{ \pm} u_{1} u_{2}\right)_{j m}^{i n}-\frac{Q_{+}^{2}}{\mathfrak{s}_{ \pm}}\left(P_{ \pm}\left(v_{ \pm}\right)_{2} P_{ \pm}\right)_{j m}^{i n}+\frac{1+q^{2}}{\mathfrak{s}_{ \pm} \mathfrak{t}_{ \pm}}\left(P_{ \pm}\right)_{j m}^{i n} V_{ \pm} .
$$


(Here, as usual, lower indices refer to the corresponding places of the tensor product; for instance, $\left(P_{ \pm}\left(v_{ \pm}\right)_{2} P_{ \pm}\right)_{j m}^{i n}$ stands for $\left(P_{ \pm}\right)_{k l}^{i n}\left(v_{ \pm}\right)_{r}^{l}\left(P_{ \pm}\right)_{j m}^{k r}$. It is easily seen that $S_{+}$and $S_{-}$are well-defined. In the proof of Lemma 4.1 we show that $S_{+}$and $S_{-}$are projections, i.e. $S_{ \pm}^{2}=S_{ \pm}$. Note that the numbers $\mathfrak{s}_{\mathfrak{s}_{+}}, \mathfrak{s}_{-}, \mathfrak{t}_{+}$and $t$ - are non-zero, since we assume that $q$ is not a root of unity.)

We denote the $N$ complex roots of $q^{2}$ by $q_{1}, \ldots, q_{N}$. Let $\mathscr{Q}^{ \pm}$be the set of all $q \in \mathbb{C}, q \neq 0$, for which $\left(\mathfrak{s}_{ \pm}^{\prime}\right)^{N}=q^{ \pm 2} \mathfrak{s}^{N}$. For each $q \in \mathscr{Q}^{ \pm}$there is a unique $r \in$ $\{1, \ldots, N\}$ such that $q_{r}^{\mp 1} \mathfrak{s}_{ \pm}^{\prime}=\mathfrak{s}$. We denote this index $r$ by $r( \pm, q)$. For notational simplicity we set $r( \pm, q)=0$ if $q \notin \mathscr{Q}^{ \pm}$.

Let $k \in\{1, \ldots, N\}$. We define

$$
\begin{aligned}
& \lambda_{+, k}^{+}:=\mathfrak{s}_{+} Q_{+}^{-1} q q_{k}^{-1}, \quad \lambda_{+, k}^{-}:=\mathfrak{s}_{-} Q_{+}^{-1} q^{-1} q_{k}^{-1}, \\
& \mu_{+, k}^{+}:=\frac{\mathfrak{t}_{+}\left(\mathfrak{s}-q^{-2}+q^{2}\right) q_{k}^{-2}-\mathfrak{t}_{+} \mathfrak{s}}{q Q_{+}\left(q_{k}^{-1} \mathfrak{s}_{+}^{\prime}-\mathfrak{s}\right)}, \quad \mu_{+, k}^{-}:=\frac{\mathfrak{t}_{-}\left(\mathfrak{s}-q^{-4}+1\right) q_{k}^{-2}-\mathfrak{t}_{-} \mathfrak{s}}{q Q_{+}\left(q_{k}^{-1} \mathfrak{s}_{+}^{\prime}-\mathfrak{s}\right)},
\end{aligned}
$$

if $k \neq r(+, q)$, and

$$
\begin{aligned}
& \lambda_{-, k}^{+}:=\mathfrak{s}_{+} Q_{+}^{-1} q^{-1} q_{k}, \quad \lambda_{-, k}^{-}:=\mathfrak{s}_{-} Q_{+}^{-1} q q_{k}, \\
& \mu_{-, k}^{+}:=\frac{\mathfrak{t}_{+}\left(\mathfrak{s}-q^{-2 N}+q^{-2 N-4}\right) q_{k}^{2}-\mathfrak{t}_{+} \mathfrak{s}}{q Q_{+}\left(q_{k} \mathfrak{s}_{-}^{\prime}-\mathfrak{s}\right)}, \\
& \mu_{-, k}^{-}:=\frac{\mathfrak{t}_{-}\left(\mathfrak{s}-q^{-2 N+2}+q^{-2 N-2}\right) q_{k}^{2}-\mathfrak{t}_{-} \mathfrak{s}}{q Q_{+}\left(q_{k} \mathfrak{s}_{-}^{\prime}-\mathfrak{s}\right)},
\end{aligned}
$$

if $k \neq r(-, q)$.

Suppose that $\tau \in\{+,-\}, k \in\{1, \ldots, N\}$ and $k \neq r(\tau, q)$. Let $\mathscr{B}(\tau, k)$ be the linear subspace of $\tilde{\mathscr{A}}_{1} \oplus \tilde{\mathscr{A}}_{2}$ generated by the following groups of elements:

(1) $S_{ \pm}\left(u_{j}^{i} u_{m}^{n}\right), i, j, n, m=1, \ldots, N$.

(2) $\left(v_{ \pm}\right)_{j}^{i}-\lambda_{\tau, k}^{ \pm} u_{j}^{i}-\delta_{i j} \mathfrak{s}^{-1}\left(V_{ \pm}-\lambda_{\tau, k}^{ \pm} U\right), \quad i, j=1, \ldots, N$.

(3) $\tilde{V}_{ \pm}^{ \pm}-\mu_{\tau, k}^{ \pm} \tilde{U}$.

(From Lemmas 4.1 and 4.2 below it follows that these elements span indeed six ad-invariant linear subspaces of ker $\varepsilon$.)

The right ideals $\mathscr{R}(\tau, k):=\mathscr{B}(\tau, k) \cdot \mathscr{A}$ of the algebra ker $\varepsilon$ are crucial for our classification of bicovariant differential calculi on the quantum group $S L_{q}(N)$. They are characterized by the following

Theorem 2.1. For each right ideal $\mathscr{R}$ of the algebra ker $\varepsilon$ the following statements are equivalent:

(i) $\mathscr{R}$ is ad-invariant, $\operatorname{ker} \varepsilon=\mathscr{R}+\tilde{\mathscr{A}}_{1}$ and $\operatorname{codim} \mathscr{R} \geqq 2$.

(ii) $\mathscr{R}=\mathscr{R}(\tau, k)$ for some $\tau \in\{+,-\}, k \in\{1, \ldots, N\}, k \neq r(\tau, q)$.

Furthermore, we have $\operatorname{ker} \varepsilon=\mathscr{R}(\tau, k) \oplus \tilde{\mathscr{A}}_{1}$ and the right ideals $\mathscr{R}(\tau, k)$ are mutually different.

The Bicovariant Differential Calculi $(\Gamma( \pm, k), d)$

Suppose that $k \in\{1, \ldots, N\}$. We introduce linear mappings $T( \pm, k) \in$ $L\left(\mathbb{C}^{N} \otimes \mathbb{C}^{N} \otimes \mathbb{C}^{N}\right)$ by

$$
\begin{aligned}
& T(+, k):=q_{k}^{-1} X(+, k)_{23}^{-1} \hat{R}_{12} \hat{R}_{23} X(+, k)_{12} \quad \text { if } k \neq r(+, q) \quad \text { and } \\
& T(-, k):=q_{k} X(-, k)_{23}^{-1} \hat{R}_{12}^{-} \hat{R}_{23}^{-1} X(-, k)_{12} \quad \text { if } k \neq r(-, q),
\end{aligned}
$$


where $\hat{R}, \hat{R}^{-}, K, X( \pm, k) \in L\left(\mathbb{C}^{N} \otimes \mathbb{C}^{N}\right)$ are defined by

$$
\begin{aligned}
\hat{R}_{j m}^{i n} & :=\hat{R}_{m n}^{j i}, \quad\left(\hat{R}^{-}\right)_{j m}^{i n}:=\left(\hat{R}^{-1}\right)_{m n}^{j i} \quad \text { and } K:=\mathfrak{s} P_{0}, \\
X(+, k) & :=I+\left(1-q_{k}\right) q Q^{-1} K \quad \text { and } X(-, k):=I+\left(q_{k}^{-1}-1\right) q^{2 N+1} Q^{-1} K .
\end{aligned}
$$

(Note that $X( \pm, k)$ is invertible and $X( \pm, k)^{-1}=I+\left(1-q_{k}^{\mp 1}\right)$ $\left(q_{k}^{\mp 1} \mathfrak{s}_{ \pm}^{\prime}-\mathfrak{s}\right)^{-1} K$, since $k \neq r( \pm, q)$.)

Let $p$ be an $N^{\text {th }}$ root of $q$ and let $L_{p}^{ \pm}=\left(\frac{ \pm}{p} l_{j}^{i}\right)$ be the $N \times N$ matrix of linear functionals ${ }_{p}^{ \pm} l_{j}^{i}$ on $\mathscr{A}$ as defined in [FRT], Sect. 2, by taking the matrix $p^{-1} P \hat{R}$ as $R$. By definition (cf. [FRT], (2.1)), we have

$$
{ }_{p}^{+} l_{j}^{i}\left(u_{m}^{n}\right)=p^{-1} \hat{R}_{m j}^{i n} \quad \text { and }{ }_{p}^{-} l_{j}^{i}\left(u_{m,}^{n}\right)=p\left(\hat{R}^{-1}\right)_{m j}^{i n}
$$

for $i, j, n, m=1, \ldots, N$. From this it follows that

$$
\kappa\left({ }_{p}^{+} l_{j}^{i}\right)\left(u_{m}^{n}\right)=p\left(\hat{R}^{-1}\right)_{j m}^{n i} \quad \text { and } \kappa\left({ }_{p}^{-} l_{j}^{i}\right)\left(u_{m}^{n}\right)=p^{-1} \hat{R}_{j m}^{n i} .
$$

Formula (2.4) in [FRT] implies that the matrices $L_{p}^{+}$and $L_{p}^{-}$define representations of the algebra $\mathscr{A}$ on $\mathbb{C}^{N}$. Let $L_{p}^{ \pm, c}$ be the contragredient representation of $L_{p}^{ \pm}$. As pointed out by B. Jurčo [J], there are four important bicovariant bimodules of the Hopf algebra $\mathscr{A}$ :

$$
{ }_{p} \Gamma_{1}=\left(u, L_{p}^{+, c}\right),{ }_{p} \Gamma_{2}=\left(u, L_{p}^{-, c}\right),{ }_{p} \Gamma_{1}^{c}=\left(u^{c}, L_{p}^{-}\right),{ }_{p} \Gamma_{2}^{c}=\left(u^{c}, L_{p}^{+}\right) .
$$

(In order to prove that these are indeed bicovariant bimodules, it only remains to check the compatibility condition (1.1). For this we apply Lemma 1.3. For ${ }_{p} \Gamma_{1}$ and ${ }_{p} \Gamma_{2}$ the corresponding mapping $T$ from Lemma 1.3 is $p^{-1} \hat{R}$, resp. $p \hat{R}^{-1}$ by $(2.5)$, so $T \in \operatorname{Mor}(u \otimes u)$. In case of $\Gamma_{1}^{c}$ and $\Gamma_{p}^{c}$ we conclude from $(2.6)$ that $T$ is $p^{-1} \hat{R}$, resp. $p R^{-}$, hence $T$ belongs to $\operatorname{Mor}\left(u^{c} \otimes u, u \otimes u^{c}\right)$ by Lemma 3.3.)

Suppose now that $p$ and $p^{\prime}$ are $N^{\text {th }}$ roots of $q$ such that $q_{k}=p p^{\prime}$. By Lemma 1.2, $\Gamma(+, k):={ }_{p} \Gamma_{2}^{c} \otimes_{p^{\prime}} \Gamma_{2}$ and $\Gamma(-, k):={ }_{p} \Gamma_{1}^{c} \otimes_{p^{\prime}} \Gamma_{1}$ are bicovariant bimodules of $\mathscr{A}$. Their structures are given as follows. The subspace $\Gamma( \pm, k)_{\mathrm{inv}}$ of left-invariant elements has a basis $\left\{\eta_{i j}: i, j=1, \ldots, N\right\}$ such that the right and left module operations of $\Gamma( \pm, k)$ satisfy the equations

$$
\eta_{i j} a=\left(\frac{ \pm}{p} l_{n}^{i} \kappa\left({ }_{p^{\prime}}^{\mp} l_{j}^{m}\right) * a\right) \eta_{n m}, \quad a \in \mathscr{A} .
$$

In both cases the set $\left\{\eta_{i j}: i, j=1, \ldots, m\right\}$ is a free basis for the corresponding left and right modules and the right action is given by $\Delta_{R}\left(\eta_{i j}\right)=\eta_{n m} \otimes\left(u^{c}\right)_{i}^{n} u_{j}^{m}$. From this and (2.3) it follows that the left-invariant element $\eta:=\sum_{i} q^{-2 i} \eta_{i i}$ is also right-invariant. For $a \in \mathscr{A}$, we define

$$
d a=\eta a-a \eta
$$

Our main results on classification of bicovariant differential calculi on $S L_{q}(N)$ are summarized in the following

Theorem 2.2. Let $(\Gamma, d)$ be a first order differential calculus on $S L_{q}(N)$. The following two assertions are equivalent:

(i) $(\Gamma, d)$ is bicovariant, $\operatorname{dim} \Gamma_{\text {inv }} \geqq 2$ and $\Gamma=\left\langle a \cdot d u_{j}^{i}: a \in \mathscr{A}, i, j=1, \ldots, N\right\rangle$.

(ii) $(\Gamma, d)$ is isomorphic to $(\Gamma(\tau, k), d)$ for some $k \in\{1, \ldots, N\}, \tau \in\{+,-\}$, $k \neq r(\tau, q)$. 
Moreover, $(\Gamma(\tau, k), d)$ is the canonical calculus associated with the ad-invariant right ideal $\mathscr{R}(\tau, k)$ of $\operatorname{ker} \varepsilon$. Two calculi $(\Gamma(\tau, k), d)$ and $\left(\Gamma\left(\tau^{\prime}, k^{\prime}\right), d\right)$ are isomorphic only if $(\tau, k)=\left(\tau^{\prime}, k^{\prime}\right)$.

The structure of $(\Gamma(\tau, k), d)$ can be also described as follows: The forms $\omega_{i j}:=$ $P_{\text {inv }}\left(d u_{j}^{i}\right)=\kappa\left(u_{n}^{i}\right) d u_{j}^{n}, i, j=1, \ldots, N$, are a basis of the vector space $\Gamma(\tau, k)_{\text {inv }}$ of left-invariant forms. In terms of this basis the bimodule structure of $\Gamma(\tau, k)$ and the differentiation $d$ of $(\Gamma(\tau, k), d)$ are given by

$$
\omega_{i j} a=\left({ }_{k}^{\tau} f_{n m}^{i j} * a\right) \omega_{n m} \text { and } d a=\left(\begin{array}{l}
\tau \\
k
\end{array} \chi_{n m} * a\right) \omega_{n m}, \quad a \in \mathscr{A},
$$

where ${ }_{k}^{\tau} f_{n m}^{i j}$ and ${ }_{k}^{\tau} \chi_{n m}$ are linear functionals on $\mathscr{A}$ such that

$$
{ }_{k}^{\tau} \chi_{n m}\left(\tilde{u}_{j}^{i}\right)=\delta_{i n} \delta_{m j} \quad \text { and }{ }_{k}^{\tau} \chi_{n m}\left(\tilde{u}_{j}^{i} u_{s}^{r}\right)={ }_{k}^{\tau} f_{n m}^{i j}\left(u_{s}^{r}\right)=T(\tau, k)_{i j s}^{r n m} .
$$

Moreover, we have $\Delta_{R}\left(\omega_{i j}\right)=\omega_{n m} \otimes\left(u^{c}\right)_{i}^{n} u_{j}^{m}$ and $d a=\vartheta_{\tau, k}(\omega a-a \omega), a \in \mathscr{A}$, where $\omega:=\sum_{i} q^{-2 i} \omega_{i i}, \vartheta_{+, k}:=\left(q_{k}^{-1} \mathfrak{s}_{+}^{\prime}-\mathfrak{s}\right)^{-1}$ and $\vartheta_{-, k}:=\left(q_{k} \mathfrak{s}_{-}^{\prime}-\mathfrak{s}\right)^{-1}$.

The proof of Theorems 2.1 and 2.2 will be given in Sect. 4. Here we continue with some remarks which are related to these results.

1. From the above formulas we compute that $\left(U-q_{k}^{\mp 1}{\mathfrak{\mathfrak { s } ^ { \prime }}}_{ \pm}\right)\left(I-P_{0}\right) x \in \mathscr{R}( \pm, k)$ for $x \in \mathscr{A}_{1}$ and $\left(U-q_{k}^{\mp 1} \mathfrak{s}_{ \pm}^{\prime}\right) \tilde{U} \in \mathscr{R}( \pm, k), k \neq r( \pm, q)$. The fact that in both expressions the same coefficient $q_{k}^{\mp 1} \mathfrak{s}_{ \pm}^{\prime}$ appears is crucial from a technical point of view, because it implies (by tracing back the proofs in Sect. 4) that the bicovariant differential calculi associated with $\mathscr{R}( \pm, k)$ are inner.

2. We briefly discuss the "critical values" of $q$ in $\mathscr{Q}^{+} \cup \mathscr{2}^{-}$. Obviously, $q \in \mathscr{Q}^{+}$if and only if $q^{-1} \in \mathscr{2}^{-}$. Apart from roots of unity, for $N=3$ and $N=4$ the set $\mathscr{Q}^{+} \cup \mathscr{Q}^{-1}$ consists of 16, resp. 32 numbers, none of them is real. For these values of $q$, there are 5 , resp. 7 bicovariant calculi in Theorem 2.2, (ii).

Suppose that $q \in \mathscr{Q}^{\tau}$ for $\tau \in\{+,-\}$ and $k=r(\tau, q)$. Then $(\Gamma(\tau, k), d)$ is still an $N^{2}$-dimensional bicovariant differential calculus over $\mathscr{A}$, but $\Gamma(\tau, k) \neq\left\langle a \cdot d u_{j}^{i}: a \in\right.$ $\mathscr{A}, i, j=1, \ldots, N\rangle$. To verify the latter, it suffices to note that the one form $\omega:=q^{-2 i} \kappa\left(u_{j}^{i}\right) d u_{i}^{j}$ is zero in $\Gamma(\tau, k)$ as computed easily, hence $\Gamma \neq\left\langle a \cdot d u_{j}^{i}\right\rangle$. However, $\Gamma(\tau, k)=\left\langle a \cdot d u_{j}^{i}, b \cdot d\left(u_{m}^{n} u_{n}^{m}\right): a, b \in \mathscr{A}, i, j=1, \ldots, N\right\rangle$ if $n \neq m$.

3. If $p$ and $p^{\prime}$ are $N^{\text {th }}$ roots of $q$ and if $r=1,2$, then the bicovariant bimodules ${ }_{p^{\prime}} \Gamma_{r} \otimes_{p} \Gamma_{r}^{c}$ and ${ }_{p} \Gamma_{r}^{c} \otimes_{p^{\prime}} \Gamma_{r}$ and hence the corresponding inner bicovariant differential calculi over $\mathscr{A}$ are isomorphic. If $\left\{\zeta_{m n}\right\}$ is the standard basis of $\left(p_{p^{\prime}} \Gamma_{r} \otimes_{p} \Gamma_{r}^{c}\right)_{\mathrm{inv}}$; then the map $\left(\tilde{R}^{-}\right)_{i j}^{m n} \zeta_{m n} \rightarrow \eta_{i j}$ for $r=1$, resp. $\hat{R}_{i j}^{m n} \zeta_{m n} \rightarrow \eta_{i j}$ for $r=2$ extends to an isomorphism of ${ }_{p^{\prime}} \Gamma_{r} \otimes_{p} \Gamma_{r}^{c}$ on ${ }_{p} \Gamma_{r}^{c} \otimes_{p^{\prime}} \Gamma_{r}$. In proving the latter, one uses that $\hat{R}, R^{-} \in \operatorname{Mor}\left(u^{c} \otimes u, u \otimes u^{c}\right) \quad$ by Lemma 3.3 and that $\hat{R}^{t} \in$ $\operatorname{Mor}\left(L_{p^{\prime}}^{-, c} * L_{p}^{+}, L_{p}^{+} * L_{p^{\prime}}^{-, c}\right)$, resp. $\left(R^{-}\right)^{t} \in \operatorname{Mor}\left(L_{p^{+}}^{+, c} * L_{p}^{-}, L_{p}^{-} * L_{p^{\prime}}^{+, c}\right)$.

4. In this remark we show that up to isomorphism there are precisely $N-1$ one dimensional bicovariant differential calculi on $S L_{q}(N)$. Suppose that $\zeta$ is an $N^{\text {th }}$ root of unity and $\zeta \neq 1$. Let $f_{\zeta}$ be the multiplicative linear functional on $\mathscr{A}$ defined by $f_{\zeta}\left(u_{j}^{i}\right)=\zeta \delta_{i j}$. Clearly, $\Gamma_{\zeta}=\left(\mathbb{1}, f_{\zeta}\right)$ is a bicovariant bimodule. That is, $\Gamma_{\zeta}$ has a fre left module basis consisting of a single left- and right-invariant element $\omega_{0}$ and $\omega_{0} a=\left(f_{\zeta} * a\right) \omega_{0}, a \in \mathscr{A}$. Defining $d a:=(\zeta-1)^{-1}\left(\omega_{0} a-a \omega_{0}\right)$ for $a \in \mathscr{A},\left(\Gamma_{\zeta}, d\right)$ is obviously a one dimensional bicovariant differential calculus on $S L_{q}(N)$. Conversely, each one dimensional bicovariant differential calculus $(\Gamma, d)$ on $S L_{q}(N)$ is isomorphic to some calculus $\left(\Gamma_{\zeta}, d\right)$. 
We prove the latter assertion. Let $\mathscr{R}$ be the ad-invariant right ideal of ker $\varepsilon$ associated with $(\Gamma, d)$. Let $\omega \in \Gamma_{\mathrm{inv}}, \omega \neq 0$, and let $f$ and $\chi$ be the corresponding linear functionals on $\mathscr{A}$ such that $\omega a=(f * a) \omega$ and $d a=(\chi * a) \omega$ for $a \in \mathscr{A}$. First note that $\left(I-P_{0}\right) \mathscr{A}_{1} \cap \mathscr{R} \neq\{0\}$, since otherwise $\operatorname{codim} \mathscr{R}=\operatorname{dim} \Gamma_{\text {inv }} \geqq N^{2}-1$. Since $\mathscr{R}$ and $\left(I-P_{0}\right) \mathscr{A}_{1}$ are ad-invariant and ad $\left\lceil\left(I-P_{0}\right) \mathscr{A}_{1}\right.$ is irreducible (see Lemma 4.1, (i), below), we conclude that $\left(I-P_{0}\right) \mathscr{A}_{1} \subseteq \mathscr{R}$. Hence $\widetilde{U} \notin \mathscr{R}$, since otherwise $\tilde{\mathscr{A}}_{1}=\langle\tilde{U}\rangle+\left(I-P_{0}\right) \mathscr{A}_{1} \subseteq \mathscr{R}$ and so $\mathscr{R}=\operatorname{ker} \varepsilon$. Since $\chi$ annihilates $\mathscr{R}$, $c:=\chi(\tilde{U}) \neq 0$ (otherwise $\chi \equiv 0$ and so $d \equiv 0$ ) and $\chi\left(u_{j}^{n}\right)=\delta_{n j} \mathfrak{s}^{-1} \chi(\tilde{U})=\delta_{n j} c^{-1}$, so that $d u_{j}^{i}=\left(\chi * u_{j}^{i}\right) \omega=c_{s^{-1}} u_{j}^{i} \omega$ and $q^{-2 j} \kappa\left(u_{j}^{i}\right) d u_{j}^{i}=c s^{-1} \omega$. But the form $q^{-2 j} \kappa\left(u_{i}^{j}\right) d u_{j}^{i}$ is right-invariant by the bicovariance of the calculus $(\Gamma, d)$. Hence $\omega$ is right-invariant and the compatibility condition (1.1) yields $f\left(u_{n}^{i}\right) u_{j}^{n}=$ $\mathbb{1}\left(u_{j}^{i} * f\right)=\left(f * u_{j}^{i}\right) \mathbb{1}=u_{n}^{i} f\left(u_{j}^{n}\right)$, so $f\left(u_{j}^{i}\right)=\zeta \delta_{i j}$ for some $\zeta \in \mathbb{C}$. From $\omega u_{j}^{i}=\zeta u_{j}^{i} \omega$ and $\operatorname{det}_{q} u=\mathbb{1}$ it follows that $\zeta^{N}=1$. If $\zeta=1$, then $\omega a=a \omega$ for $a \in \mathscr{A}$ and $d\left(u_{i_{1}}^{1} \ldots u_{i_{N}}^{N}\right)=c^{-1} N u_{i_{1}}^{1} \ldots u_{i_{N}}^{N} \omega$ by the Leibniz rule, so that $d \mathbb{1}$ $=d\left(\operatorname{det}_{q} u\right)=c \mathfrak{s}^{-1} N \omega \neq 0$ which is a contradiction. Thus $\zeta \neq 1$. Setting $\omega_{0}:=c \mathfrak{s}^{-1} \omega$, we have $\omega_{0} u_{j}^{i}-u_{j}^{i} \omega_{0}=c \mathfrak{s}^{-1}(\zeta-1) u_{j}^{i} \omega=(\zeta-1) d u_{j}^{i}$. Therefore, by the Leibniz rule, $d a=(\zeta-1)^{-1}\left(\omega_{0} a-a \omega_{0}\right)$ for all $a \in \mathscr{A}$, i.e. $(\Gamma, d)$ is isomorphic to $\left(\Gamma_{\zeta}, d\right)$.

\section{Morphisms of Tensor Products}

In this section $v$ and $w$ denote representations of the quantum group $\mathscr{A}=S L_{q}(N)$, and $\left(v_{m}^{n}\right)$, resp. $\left(w_{s}^{r}\right)$ are the corresponding matrices.

Lemma 3.1. (i) Suppose $T \in \operatorname{Mor}(u \otimes v, u \otimes w)$ and $S \in \operatorname{Mor}(v \otimes u, w \otimes u)$. We define $\left(\operatorname{tr}_{1}^{1} T\right)_{m}^{n}=q^{-2 i} T_{i m}^{i n}$ and $\left(\operatorname{tr}_{2}^{2} S\right)_{m}^{n}=q^{2 i} S_{m i}^{n i}$. Then we have $\operatorname{tr}_{1}^{1} T \in \operatorname{Mor}(v, w)$ and $\operatorname{tr}_{2}^{2} S \in \operatorname{Mor}(v, w)$.

(ii) For $T \in \operatorname{Mor}\left(u^{c} \otimes u \otimes v, u \otimes u^{c} \otimes v\right)$, define $\left(\operatorname{tr}_{12} T\right)_{r s}^{n m}=q^{-2 i} T_{i i s}^{r n m}$. Then $\operatorname{tr}_{12} T \in \operatorname{Mor}\left(u^{c} \otimes v\right)$.

Proof. (i): From $\quad T \in \operatorname{Mor}(u \otimes v, u \otimes w), \quad T_{i n}^{k r} u_{j}^{i} v_{m}^{n}=u_{i}^{k} w_{s}^{r} T_{j m}^{i s}, \quad$ so $\quad$ that $T_{i n}^{k r} q^{-2 j} \kappa\left(u_{k}^{j}\right) u_{j}^{i} v_{m}^{n}=q^{-2 j} \kappa\left(u_{k}^{j}\right) u_{i}^{k} w_{s}^{r} T_{j m}^{i s}$. Hence, by (2.3), $\left(\operatorname{tr}_{1}^{1} T\right)_{n}^{r} v_{m}^{n}=w_{s}^{r}\left(\operatorname{tr}_{1}^{1} T\right)_{m}^{s}$ which means that $\operatorname{tr}_{1}^{1} T \in \operatorname{Mor}(v, w)$.

(ii): Since $T \in \operatorname{Mor}\left(u^{c} \otimes u \otimes v, u \otimes u^{c} \otimes v\right)$, we have $T_{l m n}^{i j k} \kappa\left(u_{l}^{r}\right) u_{s}^{m} v_{t}^{n}=$ $u_{l}^{i} \kappa\left(u_{j}^{m}\right) v_{n}^{k} T_{r s t}^{\operatorname{lmn}}$ for $i, j, k, r, s, t \in\{1, \ldots, N\}$. Multiplying this equation by $q^{-2 r}$, summing over $r=s$ by using (2.3) and finally multiplying from the left by $\kappa\left(u_{i}^{p}\right)$, we get $\left(\operatorname{tr}_{12} T\right)_{i n}^{j k} \kappa\left(u_{i}^{p}\right) v_{t}^{n}=\kappa\left(u_{j}^{m}\right) v_{n}^{k}\left(\operatorname{tr}_{12} T\right)_{p t}^{m n}$, so $\operatorname{tr}_{12} T \in \operatorname{Mor}\left(u^{c} \otimes v\right)$.

Lemma 3.2. (i) $\operatorname{tr}_{1}^{1} \hat{R}=q^{-1} I, \operatorname{tr}_{1}^{1}\left(\hat{R}^{-1}\right)=q^{-2 N-1} I$.

(ii) $\operatorname{tr}_{2}^{2} \hat{R}=q^{2 N+1} I, \operatorname{tr}_{2}^{2}\left(\hat{R}^{-1}\right)=q I$.

Proof. From $\hat{R} \in \operatorname{Mor}(u \otimes u)$ and Lemma 3.1, $\operatorname{tr}_{1}^{1} \hat{R} \in \operatorname{Mor}(u)$. Since $\operatorname{Mor}(u)=\mathbb{C} \cdot I$ by Schur's lemma, there exists $\alpha \in \mathbb{C}$ such that $\left(\operatorname{tr}_{1}^{1} \hat{R}\right)_{l}^{k}=\alpha \delta_{k l}$. By definition (2.1), $\left(\operatorname{tr}_{1}^{1} \hat{R}\right)_{1}^{1}=q^{-2} \hat{R}_{11}^{11}=q^{-1}$. Hence $\alpha=q^{-1}$. Similarly, $\quad\left(\operatorname{tr}_{2}^{2} \hat{R}\right)_{l}^{k}=\beta \delta_{k l} \quad$ and $\left(\operatorname{tr}_{2}^{2} \hat{R}\right)_{N}^{N}=q^{2 N} \hat{R}_{N N}^{N N}=q^{2 N+1}$, so that $\beta=q^{2 N+1}$.

Lemma 3.3. (i) Define $\Psi_{1}(T)_{i n}^{r j}:=T_{n j}^{i r}$ and $\Psi_{2}(T)_{n i}^{j r}:=q^{2 i-2 j} T_{j n}^{r i}$. The mappings $\Psi_{1}: \operatorname{Mor}(v \otimes u, u \otimes w) \rightarrow \operatorname{Mor}\left(u^{c} \otimes v, w \otimes u^{c}\right) \quad$ and $\quad \Psi_{2}: \operatorname{Mor}(u \otimes v, w \otimes u) \rightarrow$ $\operatorname{Mor}\left(v \otimes u^{c}, u^{c} \otimes w\right)$ are isomorphisms of the corresponding intertwining spaces. 
(ii) For $T \in \operatorname{Mor}(u \otimes u)$, define $(\hat{T})_{j m}^{i n}:=T_{m n}^{j i},(\grave{T})_{j m}^{i n}:=q^{2 m-2 i} T_{i j}^{n m}$ and $(\check{T})_{j m}^{i n}:=T_{n i}^{m j}$. The mappings $T \rightarrow \tilde{T}, T \rightarrow \dot{T}, T \rightarrow \check{T}$ are isomorphisms of $\operatorname{Mor}(u \otimes u)$ onto $\operatorname{Mor}\left(u^{c} \otimes u, u \otimes u^{c}\right), \quad \operatorname{Mor}\left(u \otimes u^{c}, u^{c} \otimes u\right)$, resp. $\operatorname{Mor}\left(u^{c} \otimes u^{c}\right)$. Mor $\left(u^{c} \otimes u^{c}\right)$ is spanned by the two projections $\breve{P}_{+}$and $\breve{P}_{-}$.

(iii) The mapping $T \rightarrow T \cdot \hat{R}$ is an isomorphism of $\operatorname{Mor}\left(u \otimes u^{c}, u^{c} \otimes u\right)$ onto $\operatorname{Mor}\left(u^{c} \otimes u\right)$. Mor $\left(u^{c} \otimes u\right)$ is spanned by I and $K$.

Proof. (i): The proof is given by straightforward computations. In case of $\Psi_{2}$ we use (2.3).

(ii): The assertions follow at once from (i), since $\dot{T}=\Psi_{1}(T), \grave{T}=\Psi_{2}(T)$ and $\check{T}=\Psi_{1}\left(\Psi_{1}(T)\right)$. In the latter case the first $\Psi_{1}$ refers to $v=w=u$ and the second one to $v=w=u^{c}$. From $\operatorname{Mor}(u \otimes u)=\langle I, \hat{R}\rangle=\left\langle P_{+}, P_{-}\right\rangle$we get $\operatorname{Mor}\left(u^{c} \otimes u^{c}\right)=\left\langle\check{P}_{+}, \check{P}_{-}\right\rangle$.

(iii): Recall that $\left\{e_{i j}\right\}$ is the standard basis of $\mathbb{C}^{N} \otimes \mathbb{C}^{N}$. Since $R e_{i j}=e_{j i}, i \neq j$, and $\hat{R} e_{i i}=q^{-1} e_{i i}+Q \sum_{j \geqq i} e_{j j}$ by (2.1), $R$ is invertible, so that $T \rightarrow T \cdot R$ is an isomorphism and hence $\operatorname{dim} \operatorname{Mor}\left(u^{c} \otimes u\right)=2 . \quad$ By $\quad(2.3), \quad K \in \operatorname{Mor}\left(u^{c} \otimes u\right)$, so $\operatorname{Mor}\left(u^{c} \otimes u\right)=\langle I, K\rangle$.

In case $T=\hat{R}^{-1}$ we write $\hat{R}^{-}$for $T$ and $\grave{R}^{-}$for $\grave{T}$.

Lemma 3.4. (i) $R \grave{R}^{-}=\grave{R}^{-} \hat{R}=I$.

(ii) $\left(\dot{P}_{ \pm} \dot{P}_{ \pm}\right)_{r s}^{n m} \equiv q^{2 s-2 i}\left(P_{ \pm}\right)_{j m}^{i n}\left(P_{ \pm}\right)_{i r}^{j s}=Q_{+}^{-2}\left(\delta_{n r} \delta_{m s}+\mathfrak{s}_{ \pm} q^{2 s} \delta_{n m} \delta_{r s}\right)$.

(iii) $\operatorname{tr}_{12}\left(\tilde{R}_{12} \hat{R}_{23}\right)=Q q^{-1} I+K$ and $\operatorname{tr}_{12}\left(\hat{R}_{12}^{-} \hat{R}_{23}^{-1}\right)=Q q^{-2 N-1} I+K$.

(iv) $\operatorname{tr}_{12} T(+, k)=\left(q_{k}^{-1} \mathfrak{s}_{+}^{\prime}-\mathfrak{s}\right) I+K$ if $k \neq r(+, q)$ and $\operatorname{tr}_{12} T(-, k)=$ $\left(q_{k} \mathfrak{s}^{\prime}-\mathfrak{s}\right) I+K$ if $k \neq r(-, q)$.

(v) $\operatorname{tr}_{1}^{1}\left(T(+, k) \grave{R}_{12}\right)=\left(q_{k}^{-1}\left(q+q^{-3}\right)-q^{-1}\right) I+\xi_{+} K$ and $\operatorname{tr}_{1}^{1}\left(T(-, k) \grave{R}_{12}\right)=$ $\left(q_{k}\left(q^{-1}+q^{-2 N-1}\right)-q^{-1}\right) I+\xi_{-} K$ for some $\xi_{ \pm} \in \mathbb{C}$.

Proof. (i): By Lemma 3.3, (ii), $\dot{R}^{-} \in \operatorname{Mor}\left(u \otimes u^{c}, u^{c} \otimes u\right)$ and so $\dot{R}^{-} \hat{R} \in \operatorname{Mor}\left(u^{c} \otimes u\right)$. Hence $\grave{R}^{-} \hat{R}=\alpha I+\beta K$ with $\alpha, \beta \in \mathbb{C}$. If $i \neq j$, then $\hat{R} e_{i j}=e_{j i}$ and $\grave{R}^{-} e_{j i}=e_{i j}$ by (2.1), since $\hat{R}^{-1}=\hat{R}-Q I$. Therefore, $\alpha=1$. From $\hat{R} e_{N N}=q e_{N N}$ and $\grave{R}^{-} e_{N N}=q^{-1} e_{N N}$ again by (2.1), $\beta=0$. Thus $\dot{R}^{-} \hat{R}=I$.

(ii): Since $Q_{+} P_{+}=\hat{R}+q^{-1} I=\hat{R}^{-1}+q I$ and $Q_{+} P_{-}=-\hat{R}+q I=\hat{R}^{-1}+$ $q^{-1} I$ by (2.2), (ii) follows at once from (i) and Lemma 3.2, (i).

(iii) is obtained from Lemma 3.2, (i).

(iv): Let $\beta \in \mathbb{C}$ be such that $1+\beta \mathfrak{s} \neq 0$. Then $X:=I+\beta K$ is invertible in $L\left(\mathbb{C}^{N} \otimes \mathbb{C}^{N}\right)$ and $X^{-1}=I+\gamma K$, where $\gamma:=-\beta(1+\beta \mathfrak{s})^{-1}$. In particular, if $k \neq r( \pm, q)$, then $X( \pm, k) \quad$ is invertible and $X( \pm, k)^{-1}=I+$ $\left(1-q_{k}^{\mp 1}\right)\left(q_{k}^{\mp} \mathfrak{s}_{ \pm}^{\prime}-\mathfrak{s}\right)^{-1} K$.

We carry out the proof for $T(+, k)$. Set $\beta:=q Q^{-1}\left(1-q_{k}\right)$ and $X:=X(+, k)$. Using that $\hat{R} \dot{R}^{-}=I$ by (i), and Lemma 3.2, (i), it follows that

$$
\begin{aligned}
\left(\operatorname{tr}_{12} T(+, k)\right)_{r s}^{m n} & =q_{k}^{-1}\left(X^{-1}\right)_{i j}^{m n} \hat{R}_{l x}^{r i} \hat{R}_{y s}^{x j} X_{p p}^{l y} q^{-2 p} \\
& =q^{-1}\left(X^{-1}\right)_{i j}^{m n} \hat{R}_{l x}^{r i}\left(\left(\hat{R}^{-1}\right)_{y s}^{x j}+Q \delta_{x y} \delta_{j s}\right)(1+\beta \mathfrak{s}) q^{-2 l} \delta_{l y} \\
& =q_{k}^{-1}(1+\beta \mathfrak{s})\left(X^{-1}\right)_{i j}^{m n}\left(\hat{R}_{y x}^{r i}\left(\hat{R}^{-}\right)_{s j}^{y x} q^{-2 j}+Q \hat{R}_{y i}^{y r} q^{-2 y} \delta_{j s}\right) \\
& =q_{k}^{-1}(1+\beta \mathfrak{s})\left(\left(X^{-1}\right)_{i i}^{m n} q^{-2 i} \delta_{r s}+Q q^{-1}\left(X^{-1}\right)_{r s}^{m n}\right) \\
& =q_{k}^{-1}(1+\beta \mathfrak{s})\left(Q q^{-1} I+\left(I+\gamma \mathfrak{s}+\gamma Q q^{-1}\right) K\right)_{r s}^{m n} .
\end{aligned}
$$

Inserting the values of $\beta$ and $\gamma$, we get $\operatorname{tr}_{12} T(+, k)=\left(q_{k}^{-1} \mathfrak{s}_{+}^{\prime}-\mathfrak{s}\right) I+K$.

(v) can be derived by similar computations. 
The mappings $T( \pm, k)$ belong to the six dimensional vector space $\operatorname{Mor}\left(u^{c} \otimes u \otimes u, \quad u \otimes u^{c} \otimes u\right)$. The sets $\left\{\hat{R}_{12} \hat{R}_{23}, K_{23} \hat{R}_{12}, I_{12} \hat{R}_{23}, K_{23} I_{12}\right.$, $\left.\hat{R}_{12}, \tilde{I}_{12}\right\}$ and $\left\{\hat{R}_{12}^{-} \hat{R}_{23}^{-1}, K_{23} \hat{R}_{12}^{-}, \hat{I}_{12} \hat{R}_{23}, K_{23} \hat{I}_{12}, \hat{R}_{12}, \hat{I}_{12}\right\}$ both form a basis of this space, see Fig. 1 below. The mappings $T( \pm, k), k \neq r( \pm, q)$, can be written as follows:

$$
\begin{aligned}
T(+, k)= & q_{k}^{-1}\left(\hat{R}_{12} \hat{R}_{23}+\varphi_{+, k} K_{23} \hat{R}_{12}+\psi_{+, k} K_{23} \tilde{I}_{12}\right. \\
& \left.+\left(1-q_{k}\right) \tilde{I}_{12}\right) \\
T(-, k)= & q_{k}\left(\hat{R}_{12}^{-} \hat{R}_{23}^{-1}+\varphi_{-, k} K_{23} \hat{R}_{12}^{-}+\psi_{-, k} K_{23} \dot{I}_{12}\right. \\
& \left.+\left(1-q_{k}^{-1}\right) \tilde{I}_{12}\right),
\end{aligned}
$$

where

$$
\begin{aligned}
& \varphi_{ \pm, k}:= \pm Q\left(1-q_{k}^{\mp 1}\right)\left(q_{k}^{\mp 1} \mathfrak{s}_{ \pm}^{\prime}-\mathfrak{s}\right)^{-1} \text { and } \\
& \psi_{ \pm, k}:=\left(2-q_{k}-q_{k}^{-1}\right)\left(q_{k}^{\mp 1} \mathfrak{s}_{ \pm}^{\prime}-\mathfrak{s}\right)^{-1} .
\end{aligned}
$$

We close this section by giving the graphical representations of some important intertwiners, operations and formulas occurring in Part I of this paper. Using these graphical interpretations most of the above formulas and some of the proofs will be more transparent. In order to distinguish the places for the representations $u$ and $u^{c}$ we use arrows in the graphs. A vertex stands for $u$, resp. $u^{c}$ if the corresponding edge is downward directed, resp. upward directed. Vertices of undirected edges denote general representations.

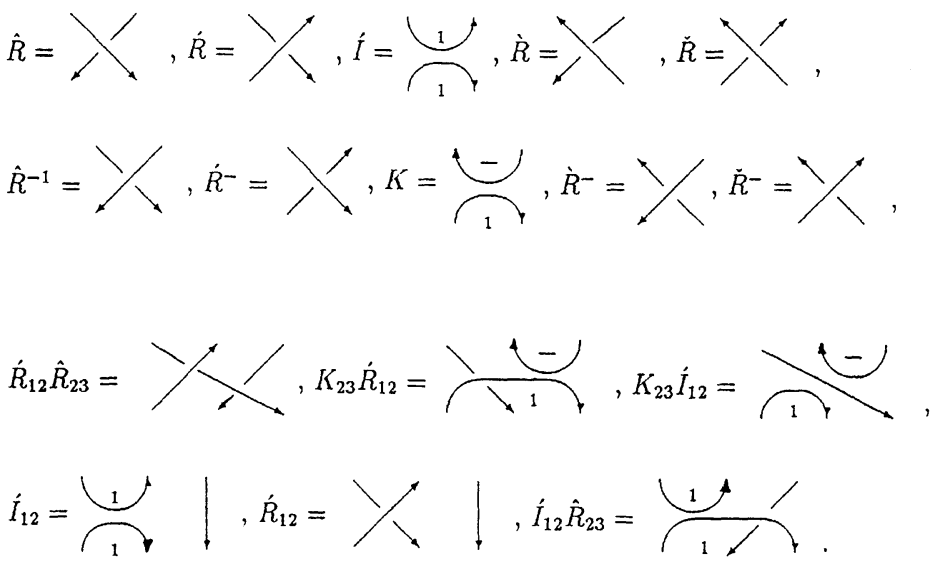

Fig. 1. The graphical representations of some intertwiners for $\mathscr{A}=S L_{q}(N)$

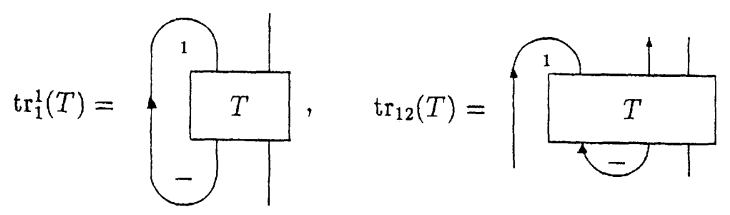

Fig. 2. The graphical representations of some operations with intertwiners for $\mathscr{A}=S L_{q}(N)$ 


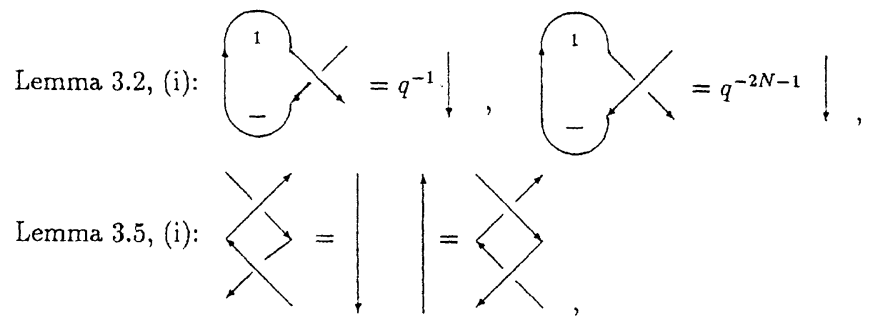

Lemma 4.6, (ii):

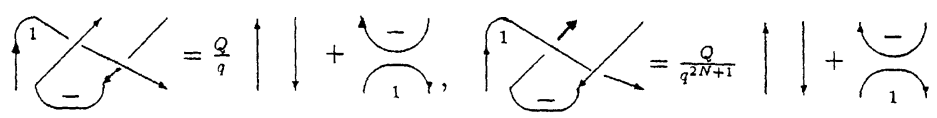

Fig. 3. The graphical representations of some identities of intertwiners for $\mathscr{A}=S L_{q}(N)$

\section{Proofs of Theorems 2.1 and 2.2}

\section{Proof of Theorem 2.1, $(i) \rightarrow(i i)$}

The proof will be divided into several steps as lemmas. We assume that $N \geqq 4$. At the end of this section we discuss the necessary modifications for the case $N=3$. First we determine the explicit decompositions of ad $\left\lceil\mathscr{A}_{1}\right.$ and $\operatorname{ad} \Gamma \mathscr{A}_{2}$ into irreducible subrepresentations.

Lemma 4.1. (i) $\operatorname{ad}\left\lceil\mathscr{A}_{1} \cong u^{c} \otimes u \cong[0] \oplus\left[2,1^{N-2}\right]\right.$.

The subspace of $\mathscr{A}_{1}$ for the trivial representation [0] is generated by $U$ and $P_{0}$ is the projection of $\mathscr{A}_{1}$ onto $\langle U\rangle$. The subspace for the Young pattern $\left[2,1^{N-2}\right]$ is $\left(I-P_{0}\right) \mathscr{A}_{1}=\left\langle u_{j}^{i}-\delta_{i j} \mathfrak{s}^{-1} U: i, j=1, \ldots, N\right\rangle$.

(ii) There are injective mappings $A_{ \pm} \in \operatorname{Mor}\left(\operatorname{ad}\left\lceil\mathscr{A}_{1}\right.\right.$, ad $\left\lceil\mathscr{A}_{2}\right)$ such that $A_{ \pm}\left(u_{j}^{i}\right)=$ $\left(v_{ \pm}\right)_{j}^{i}, i, j=1, \ldots, N$.

(iii) ad $\left\lceil\mathscr{A}_{2} \cong 2[0] \oplus 2\left[2,1^{N-2}\right] \oplus\left[4,2^{N-2}\right] \oplus\left[2,2,1^{N-4}\right]\right.$.

The corresponding subspaces of $\mathscr{A}_{2}$ are $\left\langle V_{+}\right\rangle$and $\left\langle V_{-}\right\rangle$for the trivial representation $[0]$ and $\left\langle\left(I-P_{0}\right)\left(v_{+}\right)_{j}^{i}\right\rangle$ and $\left\langle\left(I-P_{0}\right)\left(v_{-}\right)_{j}^{i}\right\rangle$ for the representation $\left[2,1^{N-2}\right]$. The projections corresponding to the Young patterns $\left[4,2^{N-2}\right]$ and $\left[2,2,1^{N-4}\right]$ are the mappings $S_{+}$and $S_{-}$, respectively, defined by (2.4).

Proof. (i): Since the contragredient representation $u^{c}$ corresponds to the Young tableau $\left[1^{N-1}\right]$ consisting of $N-1$ boxes in one column, we have $u^{c} \otimes u \cong[0] \oplus$ $\left[2,1^{N-2}\right]$, cf. e.g. [BR], ch. 8 , or $[\mathrm{H}]$, ch. 10. Clearly, the intertwiner $P_{0} \in$ $\operatorname{Mor}\left(u^{c} \otimes u\right)$ is the projection on the subspace for the trivial representation [0]. Since the $N^{2}$ elements $u_{j}^{i}$ are a basis of $\mathscr{A}_{1}, \mathbf{I}_{1} \in \operatorname{Mor}\left(u^{c} \otimes u\right.$, ad $\left\lceil\mathscr{A}_{1}\right)$ is an isomorphism and the assertion for ad $\Gamma \mathscr{A}_{1}$ follows.

(ii): Using that $\check{P}_{ \pm} \in \operatorname{Mor}\left(u^{c} \otimes u^{c}\right)$ by Lemma 3.3 and (2.3), we obtain

$$
\begin{aligned}
\operatorname{ad}\left(v_{ \pm}\right)_{j}^{i} & =\left(P_{ \pm}\right)_{n m}^{k i} q^{-2 k} \operatorname{ad}\left(u_{k}^{n} u_{j}^{m}\right) \\
& =q^{-2 k}\left(\check{P}_{ \pm}\right)_{i k}^{m n} u_{s_{1}}^{r_{1}} u_{s_{2}}^{r_{2}} \otimes\left(u^{c}\right)_{m}^{r_{2}}\left(u^{c}\right)_{n}^{r_{1}} u_{k}^{s_{1}} u_{j}^{s_{2}} \\
& =q^{-2 k}\left(\check{P}_{ \pm}\right)_{m n}^{r_{2} r_{1}} u_{s_{1}}^{r_{1}} u_{s_{2}}^{u_{2}} \otimes\left(u^{c}\right)_{i}^{m}\left(u^{c}\right)_{k}^{n} u_{k}^{s_{1}} u_{j}^{s_{2}} \\
& =\left(P_{ \pm}\right)_{r_{1} r_{2}}^{n m} u_{s_{1}}^{r_{1}} u_{s_{2}}^{r_{2}} \otimes\left(u^{c}\right)_{i}^{m}\left(\kappa\left(u_{n}^{k}\right) u_{k}^{s_{1}} q^{-2 k}\right) u_{j}^{s_{2}} \\
& =\left(P_{ \pm}\right)_{r_{1} r_{2}}^{n m} u_{s_{1}}^{r_{1}} u_{s_{2}}^{r_{2}} \otimes\left(u^{c}\right)_{i}^{m}\left(\delta_{n s_{1}} q^{-2 s_{1}}\right) u_{j}^{s_{2}} \\
& =\left(v_{ \pm}\right)_{s_{2}}^{m} \otimes\left(u^{c}\right)_{i}^{m} u_{j}^{s_{2}} .
\end{aligned}
$$


Hence, by (1.2), $A_{ \pm}: u_{j}^{i} \rightarrow\left(v_{ \pm}\right)_{j}^{i}$ defines intertwiners $A_{+}$and $A_{-}$of ad $\left\lceil\mathscr{A}_{1}\right.$ and ad $\left\lceil\mathscr{A}_{2}\right.$. Since obviously $\operatorname{dim}\left\langle\left(v_{ \pm}\right)_{j}^{i}\right\rangle>1, A_{+}$and $A_{-}$are injective.

(iii): $\mathrm{By}$ the rules for decomposing tensor product representations [BR], we have

$$
\left(u^{c} \otimes u^{c}\right) \otimes(u \otimes u)=\left(\left[2^{N-1}\right] \oplus\left[1^{N-2}\right]\right) \otimes\left([2] \oplus\left[1^{2}\right]\right)
$$

The projections corresponding to the Young patterns $\left[2^{N-1}\right]$ and $\left[1^{N-2}\right]$ in the first tensor factor are $\check{P}_{+}$, resp. $\check{P}_{-}$, cf. Lemma 3.3. The two projections for the representations [2] and $\left[1^{2}\right]$ in the second tensor factor are $P_{+}$and $P_{-}$, respectively. Using that $P_{+} \in \operatorname{Mor}(u \otimes u)$ and $P_{+} P_{-}=0$, we get

$$
\begin{aligned}
\mathbf{I}_{2}\left(\left(\check{P}_{+} \otimes P_{-}\right) e_{i j k l}\right) & =\mathbf{I}_{2}\left(\left(\check{P}_{+}\right)_{i j}^{n m}\left(P_{-}\right)_{k l}^{r s} e_{n m r s}\right) \\
& =\left(P_{+}\right)_{m n}^{j i}\left(P_{-}\right)_{k l}^{r s} u_{r}^{m} u_{s}^{n}=u_{m}^{j} u_{n}^{i}\left(P_{+}\right)_{r s}^{m n}\left(P_{-}\right)_{k l}^{r s}=0
\end{aligned}
$$

for $i, j, k, l=1, \ldots, N$, i.e. $\operatorname{im}\left(\check{P}_{+} \otimes P_{-}\right) \subseteq \operatorname{ker} \mathbf{I}_{2}$. A similar reasoning yields $\operatorname{im}\left(\check{P}_{-} \otimes P_{+}\right) \subseteq \operatorname{ker} \mathbf{I}_{2}$. From $\operatorname{dimim} \check{P}_{ \pm}=\operatorname{dimim} P_{ \pm}=\frac{1}{2} N(N \pm 1)$, we obtain $\operatorname{dimim}\left(\check{P}_{+} \otimes P_{-}+\check{P}_{-} \otimes P_{+}\right)=2 \frac{1}{4} N^{2}\left(N^{2}-1\right)$. Since also codim $\operatorname{ker} \mathbf{I}_{2}=$ $\operatorname{dim} \mathscr{A}_{2}=\frac{1}{2}\left(N^{4}-N^{2}\right)$, it follows that $\check{P}_{+} \otimes P_{+}+\check{P}_{-} \otimes P_{-}=\check{P}_{+} \otimes P_{+} \oplus$ $\check{P}_{-} \otimes P_{-}$and $\operatorname{im}\left(\check{P}_{+} \otimes P_{-} \oplus \check{P}_{-} \otimes P_{+}\right)=\operatorname{ker} \mathbf{I}_{2}$, i.e. $\mathbf{I}_{2}\lceil\mathscr{W}$ is an isomorphism of $\mathscr{W}:=\operatorname{im}\left(\check{P}_{+} \otimes P_{+} \oplus \check{P}_{-} \otimes P_{-}\right)$onto $\mathscr{A}_{2}$. Combined with (4.1), the latter yields

$$
\begin{aligned}
\operatorname{ad}\left[\mathscr{A}_{2}\right. & \cong\left[2^{N-1}\right] \otimes[2] \oplus\left[1^{N-2}\right] \otimes\left[1^{2}\right] \\
& \cong\left(\left[4,2^{N-2}\right] \oplus\left[2,1^{N-2}\right] \oplus[0]\right) \oplus\left(\left[2,2,1^{N-4}\right] \oplus\left[2,1^{N-2}\right] \oplus[0]\right)
\end{aligned}
$$

Let $E_{+}$and $E_{-}$denote the two projections corresponding to [2 $\left.2^{N-1}\right] \otimes[2]$ and $\left[1^{N-2}\right] \otimes\left[1^{2}\right]$ in (4.2). By the preceding, we have $E_{ \pm}=$ $\mathbf{I}_{2}\left(\check{P}_{ \pm} \otimes P_{ \pm}\right)\left(\mathbf{I}_{2} \Gamma \mathscr{W}\right)^{-1}$ and $E_{ \pm}\left(u_{j}^{i} u_{l}^{k}\right)=\left(P_{ \pm}\right)_{m n}^{i k} u_{j}^{m} u_{l}^{n}$. We explicitly compute the projections corresponding to the decomposition (4.3). For this let $\alpha_{ \pm}, \beta_{ \pm}$and $\gamma_{ \pm}$be complex numbers. The equations

$$
\begin{aligned}
& F_{ \pm}\left(u_{j}^{i} u_{l}^{k}\right)=\alpha_{ \pm}\left(P_{ \pm}\left(v_{ \pm}\right)_{2} P_{ \pm}\right)_{j l}^{i k}+\beta_{ \pm}\left(P_{ \pm}\right)_{j l}^{i k} V_{ \pm} \quad \text { and } \\
& G_{ \pm}\left(u_{j}^{i} u_{l}^{k}\right)=\gamma_{ \pm}\left(P_{ \pm}\right)_{j l}^{i k} V_{ \pm}
\end{aligned}
$$

define unambiguously linear mappings of $\mathscr{A}_{2}$ into itself. Using that $\check{P}_{ \pm} \in$ $\operatorname{Mor}\left(u^{c} \otimes u^{c}\right), P_{ \pm} \in \operatorname{Mor}(u \otimes u)$ and (1.2), one easily verifies that $F_{ \pm}$and $G_{ \pm}$are in $\operatorname{Mor}\left(\operatorname{ad}\left\lceil\mathscr{A}_{2}\right)\right.$. Applying Lemma 3.2, (i), we compute that $G_{+}$and $G_{-}$are projections if (and only if) $\gamma_{ \pm}=q Q_{+}\left(\text {st }_{ \pm}\right)^{-1}$ and that $G_{ \pm} F_{ \pm}=F_{ \pm} G_{ \pm}=0$ if $\gamma_{ \pm}=q Q_{+}(\mathfrak{s t} \pm)^{-1}$ and $\alpha_{ \pm}=-\mathfrak{s} \beta_{ \pm}$. Keeping the latter equations for $\alpha_{ \pm}, \beta_{ \pm}$, $\gamma_{ \pm}$, another computation based on Lemmas 3.2, (i), and 3.4, (ii), shows that $F_{ \pm}$is a projection if (and only if) $\alpha_{ \pm}=Q_{+}^{2} \mathfrak{s}_{ \pm}^{-1}$. We fix the constants $\alpha_{ \pm}, \beta_{ \pm}, \gamma_{ \pm}$by the preceding. Then $F_{ \pm}$and $G_{ \pm}$are projections and $\operatorname{im} G_{ \pm} \cap \operatorname{im} F_{ \pm}=\{0\}$. The same computation (based on Lemmas 3.2 and 3.4) just carried out also shows that $F_{ \pm}\left(\left(v_{ \pm}\right)_{j}^{i}\right)=\left(v_{ \pm}\right)_{j}^{i}-\mathfrak{s}^{-1} \delta_{i j} V_{ \pm}$. Hence $\operatorname{im} F_{ \pm}+\operatorname{im} G_{ \pm}=\left\langle\left(v_{ \pm}\right)_{j}^{i}: \quad i, j=\right.$ $1, \ldots, N\rangle=: \mathscr{V}_{ \pm}$and $F_{ \pm}+G_{ \pm}$is a projection of $\mathscr{A}_{2}$ onto $\mathscr{V}_{ \pm}$. Recall that $\mathscr{V}_{ \pm}$is ad-invariant and that $\operatorname{ad} \Gamma \mathscr{V}_{ \pm}$is equivalent to $\operatorname{ad}\left\lceil\mathscr{A}_{1} \cong\left[2,1^{N-2}\right] \oplus[0]\right.$ by (ii) and (i). Therefore, $F_{+}$and $F_{-}$are the projections corresponding to $\left[2,1^{N-2}\right]$ and $G_{+}$and $G_{-}$are the projections for [0]. Consequently, $S_{+}:=E_{+}-F_{+}-G_{+}$ 
and $S_{-}:=E_{-}-F_{-}-G_{-}$are the projections for the Young tableaus $\left[4,2^{N-2}\right]$ and $\left[2,2,1^{N-4}\right]$, respectively, in (4.3). One easily checks that $S_{+}$and $S_{-}$have the form given by (2.4). Using (i) and (ii) once more, it follows that the ad-invariant subspaces $\left\langle\left(I-P_{0}\right)\left(v_{ \pm}\right)_{l}^{k}\right\rangle$ correspond to the Young tableau $\left[2,1^{N-2}\right]$ and that $\left\langle V_{+}\right\rangle$and $\left\langle V_{-}\right\rangle$correspond to $[0]$ in (4.3).

From now on we suppose that $\mathscr{R}$ is as in Theorem 2.1, (i). We write $a \equiv b$ for $a, b \in \mathscr{A}$ if $a-b \in \mathscr{R}$.

Lemma 4.2. $\left(I-P_{0}\right) \mathscr{A}_{1} \cap \mathscr{R}=\{0\}$. In particular, $u_{j}^{i} \notin \mathscr{R}$ and $u_{i}^{i}-u_{j}^{j} \notin \mathscr{R}$ for $i \neq j$.

Proof. Assume to the contrary that $\left(I-P_{0}\right) \mathscr{A}_{1} \cap \mathscr{R} \neq\{0\}$. Recall that $\left(I-P_{0}\right) \mathscr{A}_{1}(\subseteq \operatorname{ker} \varepsilon)$ is the representation space for the Young pattern $\left[2,1^{N-2}\right]$. Since this representation is irreducible and $\mathscr{R}$ is ad-invariant, it follows that the space $\left(I-P_{0}\right) \mathscr{A}_{1}$ is in $\mathscr{R}$. Since $\operatorname{ker} \varepsilon=\mathscr{R}+\mathscr{A}_{1}$, this implies that $\operatorname{codim} \mathscr{R}=1$, which contradicts (i).

Lemma 4.3. There are complex numbers $\lambda^{+}, \lambda^{-}, \mu^{+}$and $\mu^{-}$such that

$$
\mathscr{R}_{12}=\delta\langle\tilde{U}\rangle \oplus\left\langle\tilde{V}_{+}-\mu^{+} \tilde{U}\right\rangle \oplus\left\langle\tilde{V}_{-}-\mu^{-} \tilde{U}\right\rangle \oplus \mathscr{W}_{+} \oplus \mathscr{W}_{-} \oplus \operatorname{im} S_{+} \oplus \operatorname{im} S_{-},
$$

where $\mathscr{W}_{ \pm}$denotes the linear span of elements $\left(v_{ \pm}\right)_{j}^{l}-\lambda^{ \pm} u_{j}^{i}-\delta_{i j} \mathfrak{s}^{-1}\left(V_{ \pm}-\lambda^{ \pm} U\right)$, $i, j=1, \ldots, N$, and $\delta$ is either 0 or 1 .

Proof. First note that it follows easily from Lemma 3.2, (i), that im $S_{+}, \operatorname{im} S_{-}$, $\mathscr{W}_{+}$and $\mathscr{W}_{-}$are indeed contained in ker $\varepsilon$. By Lemma 1.4, the mapping $x \rightarrow \tilde{x}=x-\varepsilon(x) \mathbb{1}$ is an isomorphism of $\mathscr{A}_{j}$ onto $\tilde{\mathscr{A}}_{j}$ for $j=1,2$ which intertwines the adjoint representation. Therefore, by Lemma 4.1, (i) and (iii),

$$
\begin{aligned}
\operatorname{ad}\left\lceil\left(\tilde{\mathscr{A}}_{1} \oplus \tilde{\mathscr{A}}_{2}\right)\right. & \cong \operatorname{ad}\left\lceil\left(\mathscr{A}_{1} \oplus \mathscr{A}_{2}\right)\right. \\
& \cong 3[0] \oplus 3\left[2,1^{N-2}\right] \oplus\left[4,2^{N-2}\right] \oplus\left[2,2,1^{N-4}\right] .
\end{aligned}
$$

Let $\mathscr{\mathscr { F }}_{1}$ be an ad-invariant linear subspace of $\tilde{\mathscr{A}}_{1} \oplus \tilde{\mathscr{A}}_{2}$ such that $\mathscr{R}_{12} \oplus \mathscr{\mathscr { F }}_{1}=$ $\tilde{\mathscr{A}}_{1} \oplus \tilde{\mathscr{A}}_{2}$. Since $\mathscr{R}_{12}+\tilde{\mathscr{A}}_{1}=\tilde{\mathscr{A}}_{1} \oplus \tilde{\mathscr{A}}_{2}$ by assumption, there is a canonical mapping of $\tilde{\mathscr{A}}_{1}$ onto $\mathscr{F}_{1}$ belonging to $\operatorname{Mor}\left(\operatorname{ad}\left\lceil\tilde{\mathscr{A}}_{1}, \operatorname{ad}\left\lceil\mathscr{F}_{1}\right)\right.\right.$. Since $\operatorname{ad}\left\lceil\tilde{\mathscr{A}}_{1} \cong[0] \oplus\left[2,1^{N-2}\right]\right.$, the latter implies that

$$
\operatorname{ad}\left\lceil\mathscr{F}_{1}=\delta_{0}[0] \oplus \delta_{1}\left[2,1^{N-2}\right],\right.
$$

where $\delta_{0}, \delta_{1} \in\{0,1\}$. Comparing the decomposition $\tilde{\mathscr{A}}_{1} \oplus \tilde{\mathscr{A}}_{2}=\mathscr{R}_{12} \oplus \mathscr{\mathscr { F }}_{1}$ with (4.5) and (4.6), we conclude that

$$
\operatorname{ad}\left\lceil\mathscr{R}_{12} \cong\left(3-\delta_{0}\right)[0] \oplus\left(3-\delta_{1}\right)\left[2,1^{N-2}\right] \oplus\left[4,2^{N-2}\right] \oplus\left[2,2,1^{N-4}\right] .\right.
$$

From Lemma 4.1, the corresponding subspaces of the decomposition (4.5) are $\left\langle\tilde{U}, \tilde{V}_{+}, \tilde{V}_{-}\right\rangle$for $3[0],\left\langle\left(I-P_{0}\right) u_{j}^{i}\right\rangle,\left\langle\left(I-P_{0}\right)\left(v_{+}\right)_{j}^{i}\right\rangle$ and $\left\langle\left(I-P_{0}\right)\left(v_{-}\right)_{j}^{i}\right\rangle$ for the three patterns $\left[2,1^{N-2}\right], \operatorname{im} S_{+}$for $\left[4,2^{N-2}\right]$ and im $S_{-}$for $\left[2,2,1^{N-4}\right]$. If $\delta_{1}=0$, then $\left\langle\left(I-P_{0}\right) u_{j}^{i}\right\rangle \subseteq \mathscr{R}_{12}$ by (4.7). But this contradicts Lemma 4.2, so that $\delta_{1}=1$ and $\left\langle\left(I-P_{0}\right) u_{j}^{i}\right\rangle \cap \mathscr{R}_{12}=\{0\}$. Hence we can write the two ad-invariant subspaces of $\mathscr{R}_{12}$ corresponding to $\left[2,1^{N-2}\right]$ of the form $\mathscr{W}_{+}$and $\mathscr{W}_{-}$described above. If $\tilde{U} \in \mathscr{R}_{12}$, then $\tilde{\mathscr{A}}_{1} \oplus \tilde{\mathscr{A}}_{2}=\mathscr{R}_{12}+\tilde{\mathscr{A}}_{1}=\mathscr{R}_{12} \oplus\left(I-P_{0}\right) \tilde{\mathscr{A}}_{1}$ and hence $\delta_{0}=0$, so that $\left\langle\tilde{U}, \tilde{V}_{+}, \tilde{V}_{-}\right\rangle \subseteq \mathscr{R}_{12}$ can be obviously written as in (4.4) with $\delta=1$. If $\tilde{U} \notin \mathscr{R}_{12}$, then $\delta_{0}=1$ and the two-dimensional subspace $\left\langle\tilde{U}, \tilde{V}_{+}, \tilde{V}_{-}\right\rangle \cap \mathscr{R}_{12}$ is of the form $\left\langle\tilde{V}_{+}-\mu^{+} \tilde{U}\right\rangle \oplus\left\langle\tilde{V}_{-}-\mu^{-} \tilde{U}\right\rangle$ as required in (4.4) with $\delta=0$. 
The main purpose of the following is to determine the values of the four numbers $\lambda^{ \pm}$and $\mu^{ \pm}$. For this we essentially use the fact that the images of $S_{+}$and $S_{-}$are contained in $\mathscr{R}_{12}$ together with some compatibility conditions. See the proof of Lemma 4.5 below.

For the following computations it is convenient to work with the numbers $\Lambda_{ \pm}$and $M_{ \pm}$defined by $\Lambda_{ \pm}:=\mathfrak{s}_{ \pm}^{-1} \lambda^{ \pm}$and $M_{ \pm}:=q\left(\mathfrak{s}_{ \pm} \mathrm{t}_{ \pm}\right)^{-1} \mu^{ \pm}$. Then, since $\mathscr{W}_{ \pm} \subseteq \mathscr{R}_{12}$ by Lemma 4.3, we have that

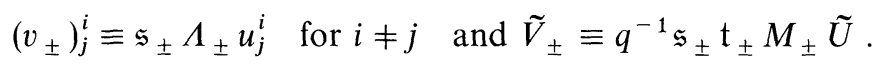

In Lemma 4.4 and in its proof we shall not sum over repeated indices.

\section{Lemma 4.4.}

(i) $u_{j}^{i} u_{l}^{k} \equiv 0$ if $i \neq j, i \neq l, k \neq j$ and $k \neq l$.

(ii) $u_{j}^{i} u_{k}^{k} \equiv\left(\Lambda_{+}+\Lambda_{-}\right) u_{j}^{i}$ if $i \neq j, i \neq k$ and $k \neq j$.

(iii) $u_{j}^{i} u_{j}^{j} \equiv u_{j}^{i} u_{i}^{i} \equiv Q_{+} \Lambda_{+} u_{j}^{i}$ if $i \neq j$.

(iv) $u_{k}^{i} u_{l}^{k} \equiv\left(q \Lambda_{+}-q^{-1} \Lambda_{-}\right) u_{l}^{i}$ if $i \neq l$ and $k<l$, $u_{k}^{i} u_{l}^{k} \equiv\left(q^{-1} \Lambda_{+}-q \Lambda_{-}\right) u_{l}^{i}$ if $i \neq l$ and $k>l$.

(v) $u_{j}^{i} u_{i}^{j} \equiv\left(q^{-1} \Lambda_{+}-q \Lambda\right) \tilde{u}_{i}^{i}+\left(q \Lambda_{+}-q^{-1} \Lambda_{-}\right) \tilde{u}_{j}^{j}$ $+\mathfrak{s}^{-1}\left(\left(\mathfrak{s}_{+} M_{+}-\mathfrak{s}_{-} M_{-}\right)-Q_{+}\left(\Lambda_{+}+\Lambda_{-}\right)\right) \tilde{U}$ if $i<j$.

(vi) $u_{i}^{i} u_{i}^{i} \equiv Q_{+}^{2} \Lambda_{+} \tilde{u}_{i}^{i}+Q_{+} \mathfrak{s}^{-1}\left(\mathfrak{s}_{+} M_{+}-Q_{+} \Lambda_{+}\right) \tilde{U}+\mathbb{1}$.

Proof. Since the sets im $S_{+}$and im $S_{-}$belong to $\mathscr{R}_{12}$ by Lemma 4.3, we have by the definition of $S_{ \pm}$,

$$
\begin{aligned}
u_{j}^{i} u_{l}^{k}-\delta_{i j} \delta_{k l} \mathbb{1}= & \left(P_{+} u_{1} u_{2}\right)_{j l}^{i k}+\left(P_{-} u_{1} u_{2}\right)_{j l}^{i k}-\delta_{i j} \delta_{k l} \mathbb{1} \\
\equiv & \left.\left.\frac{Q_{+}^{2}}{\mathfrak{s}_{+}}\left(P_{+}\left(v_{+}\right)_{2} P_{+}\right)_{j l}^{i k}\right)^{\sim}+\frac{Q_{+}^{2}}{\mathfrak{s}_{-}}\left(P_{-}\left(v_{-}\right)_{2} P_{-}\right)_{j l}^{i k}\right)^{\sim} \\
& -\frac{1+q^{2}}{\mathfrak{s}_{+} \mathfrak{t}_{+}}\left(P_{+}\right)_{j l}^{i k} \tilde{V}_{+}-\frac{1+q^{2}}{\mathfrak{s}_{-} \mathrm{t}_{-}}\left(P_{-}\right)_{j l}^{i k} \tilde{V}_{-} .
\end{aligned}
$$

The lemma will be proved by calculating the right-hand side of the preceding equation combined with (4.8). As a sample, we prove (vi). From (2.1) and (2.2) it follows that a term $\left(P_{\tau}\right)_{k m}^{i i}\left(v_{\tau}\right)_{n}^{m}\left(P_{i i}\right)_{i i}^{k n}$ is non-zero only if $\tau=+$ and $k=m=n=i$. Since $\left(P_{+}\right)_{i i}^{i i}=1$ and $\left(P_{-}\right)_{i i}^{i i}=0, \quad u_{i}^{i} u_{i}^{i}-\mathbb{1} \equiv Q_{+}^{2} \mathfrak{s}_{+}^{-1}\left(v_{+}\right)_{i}^{i}-q Q_{+}\left(\mathfrak{s}_{+} \mathrm{t}_{+}\right)^{-1} \tilde{V}$. From $\left(v_{+}\right)_{i}^{i}-\mathfrak{s}^{-1} V \equiv \lambda^{+}\left(u_{i}^{i}-\mathfrak{s}^{-1} U\right)$ we derive $\left(\tilde{v}_{+}\right)_{i}^{i} \equiv \mathfrak{s}_{+} \Lambda_{+} \tilde{u}_{i}^{i}+$ $\mathfrak{s}_{+} \mathfrak{s}^{-1}\left(q^{-1} t_{+} M_{+}-\Lambda_{+}\right) \tilde{U}$. Putting this into the preceding equation, we have $u_{i}^{i} u_{i}^{i}-\mathbb{1} \equiv Q_{+}^{2} \Lambda_{+} \tilde{u}_{i}^{i}+Q_{+\mathfrak{s}^{-1}}\left(q^{-1} Q_{+} \mathrm{t}_{+} M_{+}-Q_{+} \Lambda_{+}\right) \tilde{U}-Q_{+} M_{+} \tilde{U}$. Since $q^{-1} Q_{+} \mathfrak{t}_{+}-\mathfrak{s}=\mathfrak{s}_{+}$, (vi) follows. We omit the details of the other computations.

Lemma 4.5. The numbers $\Lambda_{ \pm}$and $M_{ \pm}$fulfill the following four equations:

(i) $1=Q_{+}^{2} \Lambda_{+}^{2}\left(\Lambda_{+}+\Lambda_{-}\right)^{N-2}$.

(ii) $0=\left(q^{-1} \Lambda_{+}-q \Lambda_{-}\right)\left(q \Lambda_{+}-q^{-1} \Lambda_{-}\right)$.

(iii) $0=-\left(\Lambda_{+}+\Lambda_{-}\right)^{2}+Q_{+}^{2} \Lambda_{+}\left(\Lambda_{+}+\Lambda_{-}+1\right)$

$$
+Q_{+} \mathfrak{s}^{-1}\left(\mathfrak{s}_{+} M_{+}-Q_{+} \Lambda_{+}\right)\left(\mathfrak{s}_{+} \Lambda_{+}+\mathfrak{s}_{-} \Lambda_{-}-\mathfrak{s}\right)+1 .
$$

(iv) $0=\left(q^{-1} \Lambda_{+}-q \Lambda_{-}\right)\left(q Q_{+} \Lambda_{+}-1\right)+\left(q \Lambda_{+}-q^{-1} \Lambda_{-}\right)\left(q^{-1} Q_{+} \Lambda_{+}-1\right)$ $+\mathfrak{s}^{-1}\left(\mathfrak{s}_{+} M_{+}-\mathfrak{s}_{-} M_{-}-Q_{+}\left(\Lambda_{+}-\Lambda_{-}\right)\right)\left(\mathfrak{s}_{+} \Lambda_{+}+\mathfrak{s}_{-} \Lambda_{-}-\mathfrak{s}\right)$. 
Proof. (i): As usual, we set $E^{i_{1} \ldots i_{N}}:=(-q)^{\sigma\left(i_{1}, \ldots, i_{N}\right)}$ if $\left(i_{1}, \ldots, i_{N}\right)$ is a permutation of the integers $1, \ldots, N$ and $\sigma\left(i_{1}, \ldots, i_{N}\right)$ denotes its length and $E^{i_{1} \ldots i_{N}}:=0$ otherwise. Using the fact that the quantum determinant $\operatorname{det}_{q} u$ is one, $u_{2}^{1} u_{i_{1}}^{1} \equiv 0$ if $i_{1}>1$ and $u_{2}^{1} u_{1}^{1} \equiv Q_{+} \Lambda_{+} u_{2}^{1}$ by Lemma 4.4, (i) and (iii), we get

$$
\begin{aligned}
u_{2}^{1} & =u_{2}^{1} u_{l_{1}}^{1} u_{i_{2}}^{2} \ldots u_{i_{N}}^{N} E^{i_{1} \ldots i_{N}} \equiv u_{2}^{1} u_{1}^{1} u_{i_{2}}^{2} \ldots u_{i_{N}}^{N} E^{1 i_{2} \ldots i_{N}} \\
& \equiv Q_{+} \Lambda_{+} u_{2}^{1} u_{i_{2}}^{2} \ldots u_{i_{N}}^{N} E^{1 i_{2} \ldots i_{N}} .
\end{aligned}
$$

If $i_{k}=2$ for some $k \geqq 3$, then we have $u_{2}^{1} u_{i_{2}}^{2} \ldots u_{2}^{k} \ldots u_{l_{N}}^{N}=$ const. $u_{2}^{1} u_{2}^{k} u_{i_{2}}^{2} \ldots u_{t_{N}}^{N} \equiv 0$ again by Lemma 4.4, (i). Therefore, by the preceding and by using $u_{2}^{1} u_{2}^{2} \equiv Q_{+} \Lambda_{+} u_{2}^{1}$, we obtain

$$
u_{2}^{1} \equiv Q_{+} \Lambda_{+} u_{2}^{1} u_{2}^{2} u_{i_{3}}^{3} \ldots u_{i_{N}}^{N} E^{12 i_{3} \ldots i_{N}} \equiv Q_{+}^{2} \Lambda_{+}^{2} u_{2}^{1} u_{i_{3}}^{3} \ldots u_{i_{N}}^{N} E^{12 l_{3} \ldots i_{N}} .
$$

A repeated application of Lemma 4.4 , (ii), leads to $u_{2}^{1} \equiv Q_{+}^{2} \Lambda_{+}^{2}\left(\Lambda_{+}+\right.$ $\left.\Lambda_{-}\right)^{N-2} u_{2}^{1} E^{12 \ldots N}$. Since $u_{2}^{1} \notin \mathscr{R}$ by Lemma 4.2 , (i) follows.

In the rest of the proof of Lemma 4.3 we will not sum over repeated indices. (ii): Suppose that $i<j<k$. By Lemma 4.4, (iv.1), $\left(q \Lambda_{+}-q^{-1} \Lambda_{-}\right) u_{k}^{i} u_{i}^{k} \equiv$ $u_{j}^{i} u_{k}^{j} u_{i}^{k}=u_{i}^{k} u_{j}^{i} u_{k}^{j} \equiv\left(q \Lambda_{+}-q^{-1} \Lambda_{-}\right) u_{j}^{k} u_{k}^{j}$. Applying now Lemma 4.4, (v), twice and using that $u_{i}^{i}-u_{j}^{j} \notin \mathscr{R}$ by Lemma 4.2 , we get (ii).

(iii) and (iv) follow in a similar way. In case of (iii) we begin with $u_{j}^{j} u_{j}^{j} u_{i}^{k}=u_{i}^{k} u_{j}^{j} u_{j}^{j}$, $i<j<k$, and apply Lemma 4.4, (ii) and (vi). Treating $u_{j}^{i} u_{i}^{j} u_{j}^{i}=u_{j}^{i} u_{j}^{i} u_{i}^{j}, i<j$, by using the assertions (v), (iii) and (i) of Lemma 4.4, (iv) can be proved. We also need that $u_{i}^{k} \notin \mathscr{R}$ for (iii) and that $u_{j}^{i} \notin \mathscr{R}$ for (iv) by Lemma 4.2.

Now we solve these four equations. Because of (ii), there are only two possible cases.

Case 1: $q^{-1} \Lambda_{+}=q \Lambda_{-}$.

Then, by (i), $\left(\Lambda_{+} Q_{+} q^{-1}\right)^{N}=q^{-2}$, i.e. there is an $N^{\text {th }}$ root $q_{k}$ of $q^{2}$ such that $\Lambda_{+} Q_{+} q^{-1}=q_{k}^{-1}$. This gives $\lambda^{+}=\lambda_{+, k}^{+}$and $\lambda^{-}=\lambda_{+, k}^{-}$. We put $\Lambda_{+}=q_{k}^{-1} q Q_{+}^{-1}$ and $\Lambda_{-}=q_{k}^{-1} q^{-1} Q_{+}^{-1}$ into the equations (iii) and (iv). If $k=r(+, q)$, then $q_{k}^{-1} \mathfrak{s}_{+}^{\prime}-\mathfrak{s}=0$ and (iv) has no solutions $M_{+}, M_{-}$. Hence $k \neq r(+, q)$. But then we find that $\mu^{+}=\mu_{+, k}^{+}$and $\mu^{-}=\mu_{+, k}^{-}$.

Case 2: $q \Lambda_{+}=q^{-1} \Lambda_{-}$.

Then we have $\left(\Lambda_{+} Q_{+} q\right)^{N}=q^{2}$ by (i) and there is a $k \in\{1, \ldots, N\}$ such that $\Lambda_{+} Q_{+} q=q_{k}$. Similarly as in Case 1 it follows that $\lambda^{ \pm}=\lambda_{ \pm}^{ \pm}, k \neq r(-, q)$ and $\mu^{ \pm}=\mu_{-, k}^{ \pm}$.

Finally, we show that $\delta=0$ (see Lemma 4.3 above). Assume to the contrary that $\delta=1$. Then $\tilde{U} \in \mathscr{R}$ by (4.4), so that $\tilde{U} u_{2}^{1}=U u_{2}^{1}-\mathfrak{s} u_{2}^{1}=\left(v_{+}\right)_{2}^{1}+\left(v_{-}\right)_{2}^{1}-\mathfrak{s} u_{2}^{1} \in \mathscr{R}$ and hence $\left(\lambda^{+}+\lambda^{-}-\mathfrak{s}\right) u_{2}^{1} \in \mathscr{R}$ by (4.4). Since $u_{2}^{1} \notin \mathscr{R}, \lambda^{+}+\lambda^{-}-\mathfrak{s}=0$. Inserting the above solutions and using $k \neq r(+, q)$ in Case 1, resp. $k \neq r(-, q)$ in Case 2, the last equation leads to a contradiction. Thus $\delta=0$. Since $\delta=0$, Lemma 4.3 says that $\mathscr{R}_{12}=\mathscr{B}(\tau, k)$, where $\tau=+$ in Case 1 and $\tau=-$ in Case 2. Since $\mathscr{R}=\mathscr{R}_{12} \cdot \mathscr{A}$ by Lemma 1.1 and $\mathscr{R}(\tau, k)=\mathscr{B}(\tau, k) \cdot \mathscr{A}$ by definition, $\mathscr{R}=\mathscr{R}(\tau, k)$. This completes the proof of implication (i) $\rightarrow$ (ii) of Theorem 2.1 for $N \geqq 4$.

In case $N=3$ the representation for the Young pattern $\left[2,2,1^{N-4}\right]$ has to be omitted in the decomposition of ad $\left\lceil\mathscr{A}_{2}\right.$ in Lemma 4.1, (iii). But, since also $S_{-}=0$ for $N=3$, the rest of the proof is still valid. 
Completions of Proofs of Theorems 2.1 and 2.2.

Let $k \in\{1, \ldots, N\}$ and $\tau \in\{+,-\}$ be such that $k \neq r(\tau, q)$. First we study the pair $(\Gamma(\tau, k), d)$ in a little more detail. Set $\omega_{i j}:=Q q^{-1} q_{k}^{-1} X(+, k)_{i j}^{n m} \eta_{n m}$ for $\tau=+$ and $\omega_{i j}:=-Q q^{-2 N-1} q_{k} X(-, k)_{i j}^{n m} \eta_{n m}$ for $\tau=-$. Since $k \neq r(\tau, q), X(\tau, k)$ is invertible, hence $\left\{\omega_{i j}: i, j=1, \ldots, N\right\}$ is a basis of the vector space $\Gamma(\tau, k)_{\text {inv }}$. Using (2.8), (2.7), (2.5), (2.6) and finally Lemma 3.6, (iii), we obtain for $\tau=+$

$$
\begin{aligned}
d u_{j}^{r} & =\eta u_{j}^{r}-u_{j}^{r} \eta=q^{-2 s}\left(\eta_{s s} u_{j}^{r}-u_{j}^{r} \eta_{s s}\right) \\
& =q^{-2 s}\left(_{k}^{+} l_{n}^{s} \kappa\left({ }_{k}^{-} l_{s}^{m}\right) * u_{j}^{r}\right) \eta_{n m}-q^{-2 n} u_{j}^{r} \eta_{n n} \\
& =q^{-2 s} u_{i k}^{r+} l_{n}^{s}\left(u_{p}^{i}\right) \kappa\left({ }_{k}^{-} l_{s}^{m}\right)\left(u_{j}^{p}\right) \eta_{n m}-q^{-2 n} u_{i}^{r} \delta_{i j} \delta_{n m} \eta_{n m} \\
& =u_{i}^{r} q^{-2 s} q_{k}^{-1} \hat{R}_{p n}^{s i} \hat{R}_{s j}^{p m} \eta_{n m}-u_{i}^{r} K_{i j}^{n m} \eta_{n m} \\
& =u_{i}^{r}\left(q_{k}^{-1} q^{-2 s}\left(\hat{R}_{12} \hat{R}_{23}\right)_{s s j}^{i n m}-K_{i j}^{n m}\right) \eta_{n m} \\
& =u_{i}^{r}\left(q_{k}^{-1} \operatorname{tr}_{12}\left(\hat{R}_{12} \hat{R}_{23}\right)-K\right)_{i j}^{n m} \eta_{n m} \\
& =u_{i}^{r}\left(q_{k}^{-1} Q q^{-1} I+\left(q_{k}^{-1}-1\right) K\right)_{i j}^{n m} \eta_{n m} \\
& =Q q^{-1} q_{k}^{-1} u_{i}^{r} X(+, k)_{i j}^{n m} \eta_{n m}=u_{i}^{r} \omega_{i j} .
\end{aligned}
$$

For $\tau=-$, a similar reasoning yields

$$
d u_{j}^{r}=Q q^{-2 N-1} q_{k} u_{i}^{r} X(-, k)_{i j}^{n m} \eta_{n m}=u_{i}^{r} \omega_{i j} .
$$

Therefore, we have $P_{\text {inv }}\left(d u_{j}^{i}\right)=\kappa\left(u_{r}^{i}\right) d u_{j}^{r}=\omega_{i j}$ in both cases. Further, the latter implies that $\tilde{\Gamma}:=\left\langle x \cdot d u_{j}^{i}: x \in \mathscr{A}, i, j=1, \ldots, N\right\rangle$ contains each form $\omega_{r s}$ and so each form $\eta_{n m}$, so that $\widetilde{\Gamma}=\Gamma(\tau, k)$. From the definition (2.8) of $d$ it is obvious that $d$ satisfies the Leibniz rule, hence $(\Gamma(\tau, k), d)$ is indeed a first order differential calculus. Since $\eta$ is both left- and right-invariant, $(\Gamma(\tau, k), d)$ is bicovariant. By construction, $\operatorname{dim} \Gamma(\tau, k)_{\text {inv }}=N^{2}$. The preceding proves the implication (ii) $\rightarrow$ (i) of Theorem 2.2 .

Next we verify that $(\Gamma(\tau, k), d)$ has the properties stated in the second half of Theorem 2.2. Since the set $\left\{\omega_{i j}\right\}$ is a basis of $\Gamma(\tau, k)_{\text {inv }}$, we know from the general theory in [Wo2] that there exist linear functionals ${ }_{k}^{\tau} f_{n m}^{i j}$ and ${ }_{k}^{\tau} \chi_{n m}$ on $\mathscr{A}$ such that (2.9) is valid. Comparing (2.7) with the first formula in (2.9), we conclude that ${ }_{k}^{\tau} f_{n m}^{i j}=X(\tau, k)_{i j}^{r s}\left(X(\tau, k)^{-1}\right)_{x y}^{n m}{ }_{k}^{\tau} l_{x}^{r} \kappa\left({ }_{k}^{\tau} l_{s}^{y}\right)$. Combined with (2.5) and (2.6), this gives ${ }_{k}^{\tau} f_{n m}^{i j}\left(u_{s}^{r}\right)=T(\tau, k)_{i j s}^{r n m}$. From the second equation in (2.9) and from $P_{\mathrm{inv}}\left(d u_{j}^{i}\right)=\omega_{i j}$ we obtain ${ }_{k}^{\tau} \chi_{n m}\left(\tilde{u}_{j}^{i}\right)={ }_{k}^{\tau} \chi_{n m}\left(u_{j}^{i}\right)=\delta_{i n} \delta_{m j}$. Comparing the latter with formula (5.20) in [Wo2], we get ${ }_{k}^{\tau} \chi_{n m}\left(\tilde{u}_{j}^{i} a\right)={ }_{k}^{\tau} f_{n m}^{i j}(a), a \in \mathscr{A}$. Thus (2.10) holds. Since $K \in$ $\operatorname{Mor}\left(u^{c} \otimes u\right)$, the formula for $\Delta_{R}\left(\omega_{i j}\right)$ follows at once from the corresponding expression for $\Delta_{R}\left(\eta_{i j}\right)$. From the definitions of $X(+, k), \omega$ and $\eta$ we obtain $\omega a-a \omega=Q q^{-1} q_{k}^{-1}\left(\eta a-a \eta+\left(1-q_{k}\right) q Q^{-1} \mathfrak{s}(\eta a-a \eta)\right)=\left(q_{k}^{-1} \mathfrak{s}_{+}^{\prime}-\mathfrak{s}\right)(\eta a-$ $a \eta)=\vartheta_{+, k} d a, a \in \mathscr{A}$, for $\tau=+$. Similarly, $\omega a-a \omega=\vartheta_{-, k} d a$ for $\tau=-$.

Let $\mathscr{R}$ be the ad-invariant right ideal of ker $\varepsilon$ which is associated with the bicovariant differential calculus $(\Gamma(\tau, k), d)$. We show that $\mathscr{R}=\mathscr{R}(\tau, k)$. Since $\operatorname{codim} \mathscr{R}=\operatorname{dim} \Gamma(\tau, k)_{\text {inv }}$ and $\operatorname{ker} \varepsilon=\mathscr{R}+\tilde{\mathscr{A}}_{1}$ by Lemma $1.5, \mathscr{R}$ satisfies the conditions in Theorem 2.1, (i). Therefore, by the proof in the preceding subsection, $\mathscr{R}=\mathscr{R}\left(\tau^{\prime}, k^{\prime}\right)$ for some $k^{\prime} \in\{1, \ldots, N\}, \tau^{\prime} \in\{+,-\}, k^{\prime} \neq r\left(\tau^{\prime}, q\right)$. Set $v_{j}^{i}:=$ $q^{-2 r} \hat{R}_{s p}^{r i} u_{r}^{s} u_{j}^{p}$. From (2.10), we have

$$
{ }_{k}^{\tau} \chi_{n m}\left(\tilde{U} u_{j}^{i}\right)=q_{k}^{-2 r \tau} \chi_{n m}\left(\tilde{u}_{r}^{r} u_{j}^{i}\right)=q^{-2 r} T(\tau, k)_{r r j}^{i n m}=\left(\operatorname{tr}_{12} T(\tau, k)\right)_{i j}^{n m}
$$


and

$$
\begin{aligned}
& { }_{k}^{\tau} \chi_{n m}\left(u_{j}^{i}\right)=q^{-2 \mathrm{r}} \hat{R}_{s p}^{\mathrm{ri}}\left({ }_{k}^{\tau} \chi_{n m}\left(\tilde{u}_{\mathrm{r}}^{s} u_{j}^{p}\right)+\delta_{\mathrm{rs}} \delta_{n p} \delta_{m j}\right. \\
& =q^{2 i-2 s-2 p} \hat{R}_{s p}^{\mathrm{r} i} T(\tau, k)_{s \mathrm{r} j}^{p n m}+q^{-2 \tau} \hat{R}_{\mathrm{r} n}^{\mathrm{r} i} \delta_{m j} \\
& =\left(\operatorname{tr}_{1}^{1}\left(T(\tau, k) \grave{R}_{12}\right)\right)_{i j}^{n m}+\left(\operatorname{tr}_{1}^{1} \hat{R}_{12}\right)_{n m}^{i j},
\end{aligned}
$$

where we used $q^{2 p-2 r} \hat{R}_{s p}^{r i}=q^{2 i-2 s} \hat{R}_{s p}^{r i}$ by (2.1). Recall that $v_{j}^{i}=q\left(v_{+}\right)_{j}^{i}-$ $q^{-1}\left(v_{-}\right)_{j}^{i}$ and $U u_{j}^{i}=\left(v_{+}\right)_{j}^{i}+\left(v_{-}\right)_{j}^{i}$ by definition. Suppose that $\tau^{\prime}=+$. Then, for $i \neq j$, the elements $a_{i j}:=\left(\tilde{U}+\mathfrak{s}-q_{k^{\prime}}^{-1} \mathfrak{s}_{+}^{\prime}\right) u_{j}^{i}=\left(v_{+}\right)_{j}^{i}+\left(v_{-}\right)_{j}^{i}-\left(\lambda_{+}^{+} k^{\prime}+\lambda_{+}^{-}, k^{\prime}\right) u_{j}^{i}$ and $b_{i j}:=v_{j}^{i}-q_{k^{\prime}}^{-1}\left(q+q^{-3}\right) u_{j}^{i}=q\left(v_{+}\right)_{j}^{i}-q^{-1}\left(v_{-}\right)_{j}^{i}-\left(q \lambda_{+, k^{\prime}}^{+}-q^{-1} \lambda_{+, k^{\prime}}\right) u_{j}^{i}$ belong to $\mathscr{R} \equiv \mathscr{R}\left(+, k^{\prime}\right)$. From (4.9), (4.10), Lemma 3.4, (iv) and (v), and Lemma 3.2, (i), we get

and

$$
{ }_{k}^{+} \chi_{i j}\left(b_{i j}\right)=\left(q_{k}^{-1}-q_{k^{\prime}}^{-1}\right)\left(q+q^{-3}\right) \quad \bar{k} \chi_{i j}\left(a_{i j}\right)=q_{k} \mathfrak{s}_{-}^{\prime}-q_{k^{\prime}}^{-1} \mathfrak{s}_{+}^{\prime}
$$

$$
\bar{k} \chi_{i j}\left(b_{i j}\right)=q_{k}\left(q^{-1}+q^{-2 N-1}\right)-q_{k^{\prime}}^{-1}\left(q+q^{-3}\right) .
$$

By the general theory ( $\left[W_{0} 2\right]$, pp. 161-162), the elements $a_{i j}$ and $b_{i j}$ are annihilated by each functional ${ }_{k}^{\tau} \chi_{n m}$. Since $q$ is not a root of unity, we conclude easily from ${ }_{k}^{\tau} \chi_{i j}\left(a_{i j}\right)={ }_{k}^{\tau} \chi_{i j}\left(b_{i j}\right)=0$ that $k=k^{\prime}$ and $\tau=+=\tau^{\prime}$. In case $\tau^{\prime}=-$ we take $a_{i j}:=\left(\tilde{U}+\mathfrak{s}-q_{k^{\prime} \mathfrak{s}_{-}^{\prime}}\right) u_{j}^{i}$ and $b_{i j}:=v_{j}^{i}-q_{k^{\prime}}\left(q^{-1}+q^{-2 N-1}\right) u_{j}^{i}$ and argue in a similar way. This proves that $(\Gamma(\tau, k), d)$ is the bicovariant calculus associated with $\mathscr{R}(\tau, k)$.

In particular, the preceding paragraph implies that $\mathscr{R}(\tau, k)$ satisfies statement (i) of Theorem 2.1. Of course, the ad-invariance of $\mathscr{R}(\tau, k)$ follows also from the ad-invariance of $\mathscr{B}(\tau, k)$ (recall that the groups of elements (1)-(3) generate adinvariant subspaces) combined with Lemma 1.7 of [Wo2]. Since $\operatorname{codim} \mathscr{R}(\tau, k)=\operatorname{dim} \Gamma(\tau, k)_{\mathrm{in} v}=N^{2}, \operatorname{ker} \varepsilon=\mathscr{R}(\tau, k) \oplus \tilde{\mathscr{A}}_{1}$. Suppose $\mathscr{R}(\tau, k)=$ $\mathscr{R}\left(\tau^{\prime}, k^{\prime}\right)$. Since $u_{j}^{i} \notin \mathscr{R}(\tau, k)$ for $i \neq j$, we see from the group (2) of generators of $\mathscr{B}(\tau, k)$ that $\lambda_{\tau, k}^{+}=\lambda_{\tau^{\prime}, k^{\prime}}$, which in turn implies that $(\tau, k)=\left(\tau^{\prime}, k^{\prime}\right)$. This completes the proof of Theorem 2.1 .

If two differential calculi $(\Gamma(\tau, k), d)$ and $\left(\Gamma\left(\tau^{\prime}, k^{\prime}\right), d\right)$ are isomorphic, then by the general theory, the corresponding right ideals $\mathscr{R}(\tau, k)$ and $\mathscr{R}\left(\tau^{\prime}, k^{\prime}\right)$ are equal. Hence $(\tau, k)=\left(\tau^{\prime}, k^{\prime}\right)$. Finally, we verify the implication (i) $\rightarrow$ (ii) of Theorem 2.2. If $(\Gamma, d)$ is as in Theorem 2.2, (i), then the associated right ideal $\mathscr{R}$ is as in Theorem 2.1, (i). Hence $\mathscr{R}=\mathscr{R}(\tau, k)$, so that $(\Gamma, d)$ is isomorphic to $(\Gamma(\tau, k), d)$. The proof of Theorem 2.2 is complete.

\section{The Classical Limits}

In the previous sections the value $q=1$ is excluded by our standing assumption that $q$ is not a root of unity. In this section we consider the case $q=1$ for $N \geqq 3$ and we briefly discuss the limits of our calculi $(\Gamma( \pm, k), d)$ for $q \rightarrow 1$. Since $q=1$, the Hopf algebra $\mathscr{A}$ is isomorphic to the coordinate Hopf algebra of $S L(n)$.

Suppose that $\zeta$ is an $N^{\text {th }}$ root of unity. Let $\lambda_{\zeta}^{ \pm}:=\frac{1}{2} \zeta(N \pm 2)$. Set $\mu_{\zeta}^{ \pm}:=\frac{1}{2}(N \pm 1)(\zeta+1)$ if $\zeta \neq 1$ and $\mu_{\zeta}^{ \pm}:=N \pm 2$ if $\zeta=1$. Let $\mathscr{R}(\zeta)$ denote the right ideal $\mathscr{B}(\zeta) \cdot \mathscr{A}$ of ker $\varepsilon$, where $\mathscr{B}(\zeta)$ is the linear subspace of $\mathscr{\mathscr { A }}_{1} \oplus \tilde{\mathscr{A}}_{2}$ generated by $\operatorname{im} S_{ \pm}, \quad\left(v_{ \pm}\right)_{j}^{i}-\lambda_{\zeta}^{ \pm} u_{j}^{i}-\delta_{i j} N^{-1}\left(V_{ \pm}-\lambda_{\zeta}^{ \pm} U\right)$ for $i, j=1, \ldots, N$ and $V_{ \pm}-\mu_{\zeta}^{ \pm} \bar{U}-\frac{N}{2}(\bar{N} \pm 1) \mathbb{1}-\mu_{\zeta}^{ \pm} N \mathbb{1}$. 
Next we define a bicovariant differential calculus $(\Gamma(\zeta), d)$ over $\mathscr{A}$. First we enumerate the $N^{\text {th }}$ roots of $q^{2}$ such that $q_{k}$ belongs to the $k^{\text {th }}$ branch of the root for all $q$ and we fix $k$ such that $q_{k}^{-1} \rightarrow \zeta$ if $q \rightarrow 1$. Let $T(\zeta)$ denote the limit of the transformation $T(+, k)$ for $q \rightarrow 1$. From (3.1) we obtain that

$$
\begin{aligned}
& T(\zeta)=\zeta \hat{R}_{12} \hat{R}_{23}+(\zeta+1) \tilde{I}_{12}-(\zeta-1) N^{-1} K_{23} \tilde{I}_{12} \text { for } \zeta \neq 1 \text { and } \\
& T(1)=\hat{R}_{12} \hat{R}_{23}+2\left(N^{2}-1\right)^{-1} K_{23} \hat{R}_{12}-2\left(N^{3}-N\right)^{-1} K_{23} \dot{I}_{12},
\end{aligned}
$$

i.e. in terms of matrix elements we have

$$
\begin{aligned}
& T(\zeta)_{i j s}^{r n m}=\zeta \delta_{i n} \delta_{j m} \delta_{r s}+(\zeta-1) \delta_{i j} \delta_{r n} \delta_{m s}-(\zeta-1) N^{-1} \delta_{i j} \delta_{n m} \delta_{r s} \\
& \quad \text { for } \zeta \neq 1 \text { and } \\
& T(1)_{i j s}^{r n m}=\delta_{i n} \delta_{j m} \delta_{r s}+2\left(N^{2}-1\right)^{-1} \delta_{n m} \delta_{i s} \delta_{r j}-2\left(N^{3}-N\right)^{-1} \delta_{i j} \delta_{n m} \delta_{r s} .
\end{aligned}
$$

We consider the bicovariant differential calculus $(\Gamma(+, k), d)$ as given in the second paragraph of Theorem 2.2 (i.e. by the formulas (2.9) and (2.10) therein) and we take the limit $q \rightarrow 1$ by keeping the basis $\left\{\omega_{i j}\right\}$ of $\Gamma(+, k)_{\text {inv }}$ fixed. Then we obtain a bicovariant differential calculus $(\Gamma(\zeta), d)$ over $\mathscr{A}$ for $q=1$ which is described again by the formulas (2.9) and (2.10) if $T(+, k)$ is replaced by $T(\zeta)$. If $\chi_{n m}$ are the corresponding linear functionals from (2.10), we define $\nabla_{n m}(a):=\chi_{n m} * a$, $a \in \mathscr{A}$. Further, we set $\nabla_{n}:=\nabla_{n+1, n+1}-\nabla_{n n}, \quad N \nabla_{U}:=\sum_{n} \nabla_{n n}, \omega_{n}:=\omega_{n n}$ and $\omega:=\sum_{n} \omega_{n}$. Obviously, by (3.2), the limits of $T(-, k)$ and $(\Gamma(-, k), d)$ for $q \rightarrow 1$ are $T\left(\zeta^{-1}\right)$ and $\left(\Gamma\left(\zeta^{-1}\right), d\right)$, respectively.

Let $\mathscr{R}$ be the ad-invariant right ideal of ker $\varepsilon$ associated with the bicovariant calculus $(\Gamma(\zeta), d)$. We verify that $\mathscr{R}=\mathscr{R}(\zeta)$. Indeed, the proof in Sect. 4 till Lemma 4.5 goes through also for $q=1$, so that $\mathscr{R}$ is completely characterized by the values of the four parameters $\lambda^{ \pm}, \mu^{ \pm}$. Since the functionals $\chi_{n m}$ annihilate $\mathscr{R}$, we conclude from (2.10) and (5.1), resp. (5.2) that $\lambda^{ \pm}=\lambda_{\zeta}^{ \pm}$and $\mu^{ \pm}=\mu_{\zeta}^{ \pm}$, hence $\mathscr{R}=\mathscr{R}(\zeta)$.

It is well-known that the classical bicovariant differential calculus $\left(\Gamma_{c}, d_{c}\right)$ over $\mathscr{A}$ is associated with the right ideal $(\operatorname{ker} \varepsilon)^{2}$ of $\operatorname{ker} \varepsilon$. It is not difficult to check that $(\operatorname{ker} \varepsilon)^{2}$ is not contained in $\mathscr{R}(\zeta)$ if $\zeta \neq 1$ and that $(\operatorname{ker} \varepsilon)^{2}=\mathscr{R}(1)+\langle\tilde{U}\rangle$. The latter relation implies that the classical calculus is a quotient of the $N^{2}$ dimensional bicovariant calculus $(\Gamma(1), d)$. That is, the limit $q \rightarrow 1$ (in the sense explained above) of the bicovariant calculus $(\Gamma( \pm, k), d)$ contains the classical calculus as a quotient if and only if $q_{k}$ belongs to the first branch of the $N^{\text {th }}$ root.

From (2.9), (2.10) and (5.1), resp. (5.2), we obtain that

$$
\begin{aligned}
\omega u_{j}^{i} & =u_{j}^{i} \omega+(\zeta-1) N d u_{j}^{i}, \\
\nabla_{n m}\left(a u_{j}^{i}\right) & =a \nabla_{n m}\left(u_{j}^{i}\right)+\zeta \nabla_{n m}(a) u_{j}^{i} \quad \text { if } n \neq m \text { and } \\
\nabla_{r}\left(a u_{j}^{i}\right) & =a \nabla_{r}\left(u_{j}^{i}\right)+\zeta \nabla_{r}(a) u_{j}^{i}+(\zeta-1) N \nabla_{U}(a) u_{j}^{i}\left(\delta_{r+1, j}-\delta_{r j}\right)
\end{aligned}
$$

for $a \in \mathscr{A}, i, j, n, m=1, \ldots, N$ and $r=1, \ldots, N-1$. If $\zeta \neq 1$, then (5.3) implies that $d a=((\zeta-1) N)^{-1}(\omega a-a \omega)$ for $a \in \mathscr{A}$, i.e. the bicovariant differential calculus $(\Gamma(\zeta), d)$ is inner. The calculus $(\Gamma(1), d)$ is not inner.

Suppose now that $\zeta=1$. Then, by (5.4) and (5.5), $\nabla_{n m}$ for $n \neq m$ and $\nabla_{r}$ for $r=1, \ldots, N-1$ satisfy the "ordinary" Leibniz rule, so they correspond to leftinvariant vector fields of the Lie group $S L(n)$. Since they are obviously linearly independent, these vector fields form a basis of the Lie algebra of $S L(n)$. Setting 
$\xi_{r}:=\frac{\mathrm{r}}{N} \omega-\omega_{1}-\cdots-\omega_{\mathrm{r}}$, we have for $a \in \mathscr{A}$,

$$
\begin{aligned}
d a-\nabla_{U}(a) \omega & =\sum_{n \neq m} \nabla_{n m}(a) \omega_{n m}-\frac{1}{N} \sum_{i, j}\left(\nabla_{i i}(a)-\nabla_{j j}(a)\right) \omega_{j} \\
& =\sum_{n \neq m} \nabla_{n m}(a) \omega_{n m}+\sum_{r=1}^{N-1} \nabla_{r}(a) \xi_{r} .
\end{aligned}
$$

We consider $\Gamma_{0}:=\left\langle a \omega_{n m}, a \omega_{\mathrm{r}}: a \in \mathscr{A}, n \neq m, r=1, \ldots, N-1\right\rangle$ as a left module of $\mathscr{A}$ and define a bimodule structure by $\eta_{0} a=a \eta_{0}, \eta_{0} \in \Gamma_{0}, a \in \mathscr{A}$. Set $d_{0} a:=d a-\nabla_{U}(a) \omega$. From the preceding it follows that $\left(\Gamma_{0}, d_{0}\right)$ is isomorphic to the classical bicovariant differential calculus $\left(\Gamma_{c}, d_{c}\right)$ over $\mathscr{A}$, i.e. we have

$$
d a=d_{c} a+\nabla_{U}(a) \omega, \quad a \in \mathscr{A} .
$$

Moreover, by (5.2),

$$
\omega_{n m} u_{j}^{i}=u_{j}^{i} \omega_{n m}+2\left(N^{2}-1\right)^{-1}\left(u_{m}^{i} \delta_{n j}-N^{-1} u_{j}^{i} \delta_{n m}\right) \omega
$$

That is, if we set $\omega=0$ in the calculus $(\Gamma(1), d)$, then the bimodule $\Gamma(1)$ becomes the commutative bimodule $\Gamma_{0}$ of $\mathscr{A}$ and the mapping $d$ of $(\Gamma(1), d)$ goes into the classical exterior derivative $d_{c}$ over $\mathscr{A}$.

\section{Part II: Classification of Bicovariant Differential Calculi on $\left(O_{q}(N)\right.$ and $\left.S p_{q}(N)\right)$}

\section{Definitions and Main Results}

Throughout Part II let $\mathscr{A}$ denote the Hopf algebra of one of the quantum groups $B_{n}, C_{n}, D_{n}$ as defined in [FRT], Definition 11. Let $N=2 n+1$ for $B_{n}$ and $N=2 n$ for $C_{n}$ and $D_{n}$. Recall that $B_{n}$, resp. $D_{n}$ is the quantum group $O_{q}(N)$ and $C_{n}$ is the quantum group $S p_{q}(N)$. Let $\hat{R}$ and $C=\left(C_{j}^{i}\right)$ be the corresponding matrices and let $P_{+}, P_{-}, P_{0}$ be the three spectral projections of $\hat{R}$ defined in [FRT], 1.4. Let $B=\left(B_{j}^{i}\right)$ denote the inverse of the matrix $C$ and let $K=\left(K_{j m}^{i k}\right)$, where $K_{j m}^{i k}:=C_{k}^{i} B_{m}^{j}$. Set $Q:=q-q^{-1}$, and $i^{\prime}:=N+1-i$. Let $z:=q^{N-1}$ for $B_{n}, D_{n}$ and $z:=-q^{N+1}$ for $C_{n}$. Note that $K_{N 1}^{N 1}=q^{-1} z, K_{1 N}^{1 N}=q z^{-1}$ and $C^{2}= \pm I$. Further, $C_{j}^{i} \neq 0$ if and only if $j=i^{\prime}$. By definition (cf. [FRT], (1.9) and (1.13)), we have

$$
\begin{aligned}
\hat{R}_{j m}^{i k}= & q \delta_{i m} \delta_{j k} \delta_{i k}+\delta_{i m} \delta_{j k}\left(1-\delta_{i k}\right)\left(1+\left(q^{-1}-1\right) \delta_{i k^{\prime}}\right) \\
& +Q \theta(k-j)\left(\delta_{i j} \delta_{k m}-K_{j j^{\prime}}^{i i^{\prime}} \delta_{i k^{\prime}} \delta_{j m^{\prime}}\right)
\end{aligned}
$$

for all $i, j, k, m=1, \ldots, N$ except for the element $\hat{R}_{n+1, n+1}^{n+1, n+1}=1$ in case of $B_{n}$ and

$$
u C u^{t} B=C u^{t} B u=I \quad \text { or equivalently } \kappa\left(u_{j}^{i}\right)=C_{k}^{i} u_{k}^{m} B_{j}^{m} .
$$

The Ad-Invariant Right Ideals $\mathscr{R}_{+}$and $\mathscr{R}_{-}$

In order to define these right ideals, we introduce a number of notations. We abbreviate $Q_{+}:=q+q^{-1}, Z:=z-z^{-1}$, 
$\mathfrak{s}:=1+Z Q^{-1}$ and $D:=B^{t} C$, i.e. $D_{j}^{i}=D_{i}^{j}=B_{i}^{k} C_{j}^{k}$. Further, we set

$$
\begin{aligned}
\left(u_{ \pm}\right)_{j}^{i} & :=B_{k}^{r}\left(P_{ \pm}\right)_{s j}^{r m} C_{s}^{i} u_{m}^{k}, \quad\left(v_{ \pm}\right)_{j}^{i}:=\left(P_{ \pm}\right)_{r s}^{m i} D_{m}^{k} u_{k}^{r} u_{j}^{s}, \\
\left(f_{ \pm}\right)_{j}^{i} & :=B_{k}^{r}\left(P_{+}\right)_{s j}^{r m} C_{s}^{i}\left(v_{ \pm}\right)_{m}^{k}, \quad\left(g_{ \pm}\right)_{j}^{i}:=B_{k}^{r}\left(P_{-}\right)_{s j}^{r m} C_{s}^{i}\left(v_{ \pm}\right)_{m}^{k} \\
U & :=D_{i}^{j} u_{j}^{i} \quad \text { and } \quad V_{ \pm}:=D_{i}^{j}\left(v_{ \pm}\right)_{j}^{i} .
\end{aligned}
$$

With the coefficients

$$
\begin{array}{ll}
\alpha_{+}:=\frac{Q Q_{+}^{2}(q z-1)}{(q z+1)(z-q)\left(q^{2}-q^{-3} z^{-1}\right)}, & \alpha_{-}:=\frac{Q Q_{+}^{2}}{(z-q)\left(q^{-2}+q z^{-1}\right)}, \\
\beta_{+}:=\frac{Q Q_{+}^{2}}{\left(z+q^{-1}\right)\left(q^{2}-q^{-1} z^{-1}\right)}, & \beta_{-}:=\frac{Q Q_{+}^{2}(z+q)}{(z-q)\left(z+q^{-1}\right)\left(q^{-2}+q^{3} z^{-1}\right)}, \\
\gamma_{+}:=\frac{Q^{2} Q_{+}}{Z(z+q)\left(q-q^{-2} z^{-1}\right)}, & \gamma_{-}:=\frac{Q^{2} Q_{+}}{Z\left(z-q^{-1}\right)\left(q^{-1}+q^{2} z^{-1}\right)},
\end{array}
$$

we define linear mappings $S_{ \pm}: \mathscr{A}_{2} \rightarrow \mathscr{A}_{2}$ by

$$
\begin{aligned}
S_{ \pm}\left(u_{j}^{i} u_{m}^{k}\right):= & \left(P_{ \pm} u_{1} u_{2}\right)_{j m}^{i k}-\alpha_{ \pm}\left(P_{ \pm}\left(f_{ \pm}\right)_{2} P_{ \pm}\right)_{j m}^{i k} \\
& -\beta_{ \pm}\left(P_{ \pm}\left(g_{ \pm}\right)_{2} P_{ \pm}\right)_{j m}^{i k}-\gamma_{ \pm}\left(P_{ \pm}\right)_{j m}^{i k} V_{ \pm} .
\end{aligned}
$$

(The proof of Lemma 8.1 below shows that $S_{+}$and $S_{-}$are well-defined projections.) We introduce twelve numbers $\varphi_{+}^{ \pm}, \varphi_{-}^{ \pm}, \psi_{+}^{ \pm}, \psi_{-}^{ \pm}, \mu_{+}^{ \pm}$and $\mu_{-}^{ \pm}$by

$$
\begin{aligned}
& \psi_{+}^{+}=-\psi_{-}^{+}=\varphi_{+}^{+}-1=-\varphi_{-}^{+}-1:=Q^{-1} Q_{+}^{-1}\left(q^{3} z-q^{-3} z^{-1}\right), \\
& \varphi_{+}^{-}=-\varphi_{-}^{-}=\psi_{+}^{-}-1=-\psi_{-}^{-}-1:=Q^{-1} Q_{+}^{-1}\left(q^{-3} z-q^{3} z^{-1}\right), \\
& \mu_{+}^{+}:=1+Q^{-1}\left(q^{2} z-q^{-2} z^{-1}\right), \quad \mu_{+}^{-}:=1+Q^{-1}\left(q^{-2} z-q^{2} z^{-1}\right) \quad \text { and } \\
& \mu_{-}^{ \pm}:=-Q Z(Q Z+2 \mathfrak{s})^{-1} \mu_{+}^{ \pm} \quad \text { if } Q Z+2 \mathfrak{s} \neq 0 .
\end{aligned}
$$

For $\tau=+$ and for $\tau=-$ if $Q Z=2 \mathfrak{s} \neq 0$, let $\mathscr{R}_{\tau}$ denote the right ideal $\mathscr{B}_{\tau} \cdot \mathscr{A}$ of ker $\varepsilon$, where $\mathscr{B}_{\tau}$ is the linear subspace of $\tilde{\mathscr{A}}_{1} \oplus \tilde{\mathscr{A}}_{2}$ spanned by the following groups of elements:

(1) $S_{ \pm}\left(u_{j}^{i} u_{m}^{k}\right), \quad i, j, k, m=1, \ldots, N$.

(2) $\left(f_{ \pm}\right)_{j}^{i}-\varphi_{\tau}^{ \pm}\left(u_{+}\right)_{j}^{i}, \quad\left(g_{ \pm}\right)_{j}^{i}-\psi_{\tau}^{ \pm}\left(u_{-}\right)_{j}^{i}, \quad i, j=1, \ldots, N$.

(3) $\tilde{V}_{ \pm}^{ \pm}-\mu_{\tau}^{ \pm} \tilde{U}$.

(Note that the eight groups of elements (1)-(3) generate ad-invariant linear subspaces of ker $\varepsilon$, see Lemmas 8.1 and 8.2 below.)

Theorem 6.1. The right ideals $\mathscr{R}_{+}$and $\mathscr{R}_{-}$for $Q Z+2 \mathfrak{s} \neq 0$ are the only ad-invariant right ideals $\mathscr{R}$ of the algebra ker $\varepsilon$ such that $\operatorname{ker} \varepsilon=\mathscr{R} \oplus \tilde{\mathscr{A}}_{1}$.

The Bicovariant Differential Calculi $\left(\Gamma_{+}, d\right)$ and $\left(\Gamma_{-}, d\right)$

For $\tau=+$ and for $\tau=-$ if $Q Z+2 \mathfrak{s} \neq 0$, we define linear mappings $X_{\tau} \in$ $L\left(\mathbb{C}^{N} \otimes \mathbb{C}^{N}\right)$ and $T_{\tau} \in L\left(\mathbb{C}^{N} \otimes \mathbb{C}^{N} \otimes \mathbb{C}^{N}\right)$ by

$$
\begin{aligned}
& X_{+}:=z I-\hat{R}, \quad X_{-}:=z I-\hat{R}+2 Q^{-1} K \quad \text { and } \\
& T_{ \pm}:= \pm\left(X_{ \pm}\right)_{23}^{-1} \hat{R}_{12}^{-1} \hat{R}_{23}\left(X_{ \pm}\right)_{12} .
\end{aligned}
$$


Note that $X_{+}^{-1}=z(Z-Q)\left[(z-Q) I+\hat{R}-Q z^{-1} Z^{-1} K\right] \quad$ and $\quad X_{-}^{-1}=$ $z(Z-Q)\left[(z-Q) I+\hat{R}+\left(Q-Q_{+}^{2} z(Q Z+2 \mathfrak{s})^{-1}\right) K\right]$.

Let $L^{ \pm}=\left({ }^{ \pm} l_{j}^{i}\right)$ be the $N \times N$ matrix of linear functions ${ }^{ \pm} l_{j}^{i}$ on $\mathscr{A}$ defined in [FRT], Sect. 2, for the quantum groups $B_{n}, C_{n}, D_{n}$. By the formula (2.1) in [FRT] we have

$$
{ }^{+} l_{j}^{i}\left(u_{m}^{k}\right)=\hat{R}_{m j}^{i k} \quad \text { and } \kappa\left({ }^{-} l_{j}^{i}\right)\left(u_{m}^{k}\right)=\hat{R}_{j m}^{k i}
$$

for $i, j, k, m=1, \ldots, N$. By a similar reasoning as in case of $S L_{q}(N), \Gamma_{2}:=\left(u, L^{-, c}\right)$ and $\Gamma_{2}^{c}:=\left(u^{c}, L^{+}\right)$are bicovariant bimodules of $\mathscr{A}$. We still need another new bicovariant bimodule $\Gamma_{0}=\left(u^{c}, L\right)$. For this let $L=\left(l_{j}^{i}\right)$ be the $N \times N$ matrix of linear functionals $l_{j}^{i}$ on $\mathscr{A}$ defined by $l_{j}^{i}(\mathbb{1}):=\delta_{i j}$ and $l_{j}^{i}(a):=(-1)^{k}\left({ }^{+} l_{j}^{i}\right)(a)$ for $a \in \mathscr{A}_{k}$ and $k \in \mathbb{N}$. Since only quadratic relations for the matrix entries $u_{j}^{i}$ are involved in the definition of the algebra $\mathscr{A}$, the functionals $l_{j}^{i}$ are well-defined. Since $L^{+}$is a representation of $\mathscr{A}$ on $\mathbb{C}^{N}, L$ is also a representation of $\mathscr{A}$. To verify the compatibility condition (1.1), we apply Lemma 1.3 . If $T$ is the mapping for $\left(u^{c}, L\right)$ occurring therein, then $-T(=R)$ is the corresponding mapping for the bicovariant bimodule $\Gamma_{2}^{c}=\left(u^{c}, L^{+}\right)$, hence $T \in \operatorname{Mor}\left(u^{c} \otimes u, u \otimes u^{c}\right)$. Thus $\Gamma_{0}=\left(u^{c}, L\right)$ is indeed a bicovariant bimodule.

By Lemma 1.2, $\Gamma_{+}:=\Gamma_{2}^{c} \otimes \Gamma_{2}$ and $\Gamma_{-}:=\Gamma_{0} \otimes \Gamma_{2}$ are bicovariant bimodules of $\mathscr{A}$. By definition there is a basis $\left\{\eta_{i j}: i, j=1, \ldots, N\right\}$ of the vector space $\left(\Gamma_{+}\right)_{\text {inv }}$ such that $\Delta_{R}\left(\eta_{i j}\right)=\eta_{k m} \otimes\left(u^{c}\right)_{i}^{k} u_{j}^{m}$. From (6.2) we conclude easily that the leftinvariant element $\eta:=D_{j}^{i} \eta_{i j}$ is also right-invariant. As in case of $S L_{q}(N)$, we set $d a=\eta a-\eta a$ for $a \in \mathscr{A}$.

Theorem 6.2. $U p$ to isomorphism $\left(\Gamma_{+}, d\right)$ and $\left(\Gamma_{-}, d\right)$ for $Q Z+2 \mathfrak{s} \neq 0$ are the only $N^{2}$ dimensional bicovariant differential calculi $(\Gamma, d)$ over $\mathscr{A}$ for which $\Gamma=\left\langle a \cdot d u_{j}^{i}: a \in \mathscr{A}, i, j=1, \ldots, N\right\rangle$.

The structure of $\left(\Gamma_{+}, d\right)$ and of $\left(\Gamma_{-}, d\right)$ for $Q Z+2 \mathfrak{s} \neq 0$ is the same as given in the second part of Theorem 2.2 if the linear mapping $T(\tau, k)$ is replaced by $B_{2}^{t} T_{+} C_{1}^{t}$, the invariant form $\omega$ by $D_{j}^{i} \omega_{i j}$ and the constant $\vartheta_{\tau . k}$ by $(Q Z)^{-1}$ for $\left(\Gamma_{+}, d\right)$ and by $(Q Z+2 \mathfrak{s})^{-1}$ for $\left(\Gamma_{-}, d\right)$. Moreover, the ad-invariant right ideal $\mathscr{R}_{ \pm}$corresponds to the bicovariant calculus $\left(\Gamma_{+}, d\right)$.

The proofs of both theorems are given in Sect. 8. We add a few remarks.

1. Theorem 6.2 corrects an error in the formulation of Theorem 7 in [SS], where the bicovariant calculus $\left(\Gamma_{-}, d\right)$ is missing.

2. If $q$ satisfies the algebraic equation $Q Z+2 \mathfrak{s}=0$, only the right ideal $\mathscr{R}_{+}$and the calculus $\left(\Gamma_{+}, d\right)$ appear in Theorems 6.1 and 6.2, respectively. While $\mathscr{R}_{-}$and $T_{-}$are not defined for $Q Z+2 \mathfrak{s}=0$, the pair $\left(\Gamma_{-}, d\right)$ is still a well-defined bicovariant differential calculus, but $\Gamma_{-} \neq\left\langle a d u_{j}^{i}\right\rangle$. The condition $Q Z+2 \mathfrak{s} \neq 0$ excludes precisely 6 values of $q$ for $O_{q}(3), 8$ for $O_{q}(4)$ and 12 for $S p_{q}(4)$; none of them is real or a root of unity.

3. Let $\Gamma_{j}$ and $\Gamma_{j}^{c}, j=1,2$, be the bicovariant bimodules of $\mathscr{A}$ which are defined as in the case of $S L_{q}(N)$ by letting $p=1$. Then the bicovariant bimodules $\Gamma_{2}^{c} \otimes \Gamma_{2}$ and $\Gamma_{1}^{c} \otimes \Gamma_{1}$ (which were not isomorphic for $S L_{q}(N)$ !) and hence the corresponding inner bicovariant calculi are isomorphic for $\mathscr{A}=B_{n}, C_{n}, D_{n}$.

4. Our standing assumption $N \geqq 3$ excludes only the quantum groups $A_{1}=S L_{q}(2)$ and $C_{1}=S p_{q}(2)$. Since the Hopf algebras $S L_{q^{2}}(2)$ and $S p_{q}(2)$ are isomorphic, it suffices to discuss the case of $S L_{q}(2)$. Then the $4 D_{+-}$and $4 D_{-}$-calculi are the only 
bicovariant differential calculi satisfying condition (ii) of Theorem 2.2, cf. [St]. Their structure is also of the form described in the second half of Theorem 2.2. We set $T_{ \pm}:= \pm\left(X_{ \pm}\right)_{23}^{-1} \hat{R}_{12}^{-1} \hat{R}_{23}\left(X_{ \pm}\right)_{12}$ for the $4 D_{ \pm}$-calculus, where $X_{+}:=$ $\left(q^{2}-Q\right) I+\hat{R}$ and $X_{-}:=\left(q^{2}+Q\right) I-\hat{R}$. In particular, both calculi are inner.

\section{Morphisms of Tensor Products}

Throughout this section let $v$ and $w$ be representations of the quantum group $\mathscr{A}$. The following notation is often used in the sequel. Let $T$ be an intertwiner of two tensor products of representations of $\mathscr{A}$, where the first one has $u$ as the $m^{\text {th }}$ factor and the second one has $u$ as the $k^{\text {th }}$ factor. We set ${ }_{m}^{k} T:=B_{k}^{t} T C_{m}^{t}$. Since $u^{c}=B^{t} u C^{t}$ by (6.2), ${ }_{m}^{k} T$ intertwines the corresponding tensor products obtained if the $m^{\text {th }}$ factor of the first one and the $k^{\text {th }}$ factor of the second one are replaced by $u^{c}$. In particular, the mapping $T \rightarrow{ }_{1}^{2} T$ is a bijection of $\operatorname{Mor}(u \otimes u \otimes v, u \otimes u \otimes w)$ to $\operatorname{Mor}\left(u^{c} \otimes u \otimes v, u \otimes u^{c} \otimes w\right)$ and $T \rightarrow{ }_{1}^{1} T$ is an algebraic isomorphism of the algebras $\operatorname{Mor}(u \otimes v)$ and $\operatorname{Mor}\left(u^{c} \otimes v\right)$. Similar to the case of $S L_{q}(N)$, we have $\check{T} \in \operatorname{Mor}\left(u^{c} \otimes u^{c}\right)$ if $T \in \operatorname{Mor}(u \otimes u)$.

Lemma 7.1. Setting $D_{i j}:=B_{r}^{i} C_{r}^{j}$ and $D^{i j}:=B_{i}^{r} C_{j}^{r}$, we have $D_{i j} u_{k}^{i}\left(u^{c}\right)_{m}^{j}=D_{k m} \mathbb{1}$ and $D^{i j}\left(u^{c}\right)_{i}^{k} u_{j}^{m}=D^{k m} \mathbb{1}$

Proof. We prove the first equality. Using (6.2), we obtain $D_{i j} u_{k}^{i}\left(u^{c}\right)_{m}^{j}=$ $B_{r}^{i} C_{r}^{j} u_{k}^{j}\left(C_{x}^{m} u_{x}^{y} B_{j}^{y}\right)=\left(u^{t} B u\right)_{x}^{k} C_{x}^{m}=B_{x}^{k} C_{x}^{m} \mathbb{1}=D_{k m} \mathbb{1}$.

Lemma 7.2. (i) For $S \in \operatorname{Mor}(u \otimes v, u \otimes w)$ and $T \in \operatorname{Mor}(v \otimes u, w \otimes u)$, we define $\left(\operatorname{tr}_{1}^{1} S\right)_{m}^{k}:=D^{i j} S_{j m}^{i k}$ and $\left(\operatorname{tr}_{2}^{2} T\right)_{m}^{k}:=D_{i j} T_{m j}^{k i}$. Then we have $\operatorname{tr}_{1}^{1} S \in \operatorname{Mor}(v, w)$ and $\operatorname{tr}_{2}^{2} T \in$ $\operatorname{Mor}(v, w)$.

(ii) Let $\left(\operatorname{tr}_{12}^{c} S\right)_{r s}^{k m}:=D^{i j} S_{i j s}^{r k m}$ and $\left(\operatorname{tr}_{12} T\right)_{r s}^{k m}:=B_{i}^{r} T_{x y s}^{i k m} C_{y}^{x}$ for $S \in \operatorname{Mor}\left(u^{c} \otimes u \otimes v\right.$, $\left.u \otimes u^{c} \otimes w\right)$ and $T \in \operatorname{Mor}(u \otimes u \otimes v, u \otimes u \otimes w)$. Then $\operatorname{tr}_{12}^{c} S \in \operatorname{Mor}\left(u^{c} \otimes v, u^{c} \otimes w\right)$ and $\operatorname{tr}_{12} T \in \operatorname{Mor}(u \otimes v, u \otimes w)$. Moreover, $\operatorname{tr}_{12}^{c} T={ }_{1}^{1}\left(\operatorname{tr}_{12} S\right)$ if $T={ }_{1}^{2} S$.

Proof. The proof is similar to the proof of Lemma 3.1 if we replace $q^{-2 i} \delta_{i j}$ and $q^{2 i} \delta_{i j}$ by $D^{i j}$ and $D_{j i}$, respectively, and use Lemma 7.1 or (6.2) instead of (2.3).

Now we introduce a twist $\Phi$ (see Fig. 5 below) of the algebra $\operatorname{Mor}(u \otimes u)$ by defining $\Phi(T)_{r s}^{k m}:=B_{i}^{r} T_{s j}^{i k} C_{m}^{j}$ for $T \in \operatorname{Mor}(u \otimes u)$. Obviously, $\Phi(I)=K$ and $\Phi(K)=I$. From formula $(1.10)$ in [FRT] we obtain $\Phi(\hat{R})=\hat{R}^{-1}$. That is, $\Phi$ maps the basis $\{\hat{R}, I, K\}$ of the vector space $\operatorname{Mor}(u \otimes u)$ on the basis $\left\{\hat{R}^{-1}, K, I\right\}$. Hence $\Phi$ is a bijective mapping of $\operatorname{Mor}(u \otimes u)$. Since $\hat{R}^{-1}=\hat{R}-Q I+Q K$ (cf. [FRT], 1.4), we conclude that $\Phi(\Phi(T))=T$ for all $T \in \operatorname{Mor}(u \otimes u)$.

Set $q_{+}:=-Q(q z-1)^{-1}$ and $q_{-}:=-Q\left(q^{-1} z+1\right)^{-1}$. Recall that by the formulas in [FRT], 1.4, we have

$$
\begin{gathered}
P_{+}=Q_{+}^{-1}\left(\hat{R}+q^{-1} I+q_{+} K\right), \quad P_{-}=Q_{+}^{-1}\left(-\hat{R}+q I+q_{-} K\right), \\
P_{0}=\mathfrak{s}^{-1} K .
\end{gathered}
$$


The morphisms $\Pi_{ \pm}:=\Phi\left(\Phi\left(P_{ \pm}\right)^{2}\right) \in \operatorname{Mor}(u \otimes u)$, see Fig. 4 below, play an important role in the proof of Lemma 8.1. By direct computations based on (7.1) and the fact that the $\Phi=\Phi^{-1}$ one proves

Lemma 7.3. (i) $\left(\Pi_{ \pm}\right)_{r s}^{k m}=B_{k}^{y}\left(P_{ \pm}\right)_{x y}^{i j} D_{p}^{i}\left(P_{ \pm}\right)_{p s}^{x m} C_{j}^{r}$.

(ii) $\Pi_{ \pm}=\alpha_{ \pm}^{-1} P_{+}+\beta_{ \pm}^{-1} P_{-}+\mathfrak{s \gamma _ { \pm } ^ { - 1 }} P_{0}$.

Lemma 7.4. (i) $\operatorname{tr}_{1}^{1} \hat{R}=z I, \operatorname{tr}_{1}^{1} \hat{R}^{-1}=z^{-1} I, \operatorname{tr}_{1}^{1} I=\mathfrak{s} I, \operatorname{tr}_{1}^{1} K=I$.

(ii) $\operatorname{tr}_{1}^{1} T=\operatorname{tr}_{2}^{2} T$ for $T \in \operatorname{Mor}(u \otimes u)$.

(iii) $\operatorname{tr}_{1}^{1} P_{ \pm}=Q_{+}^{-1}\left(q^{\mp 1} \mathfrak{s} \pm z+q_{ \pm}\right) I$ and $\operatorname{tr}_{1}^{1}\left(\operatorname{tr}_{1}^{1} P_{ \pm}\right)=\gamma_{ \pm}^{-1}$.

(iv) $\operatorname{tr}_{1}^{1} \Phi\left(P_{ \pm}\right)=0$.

(v) $\operatorname{tr}_{12}\left(\hat{R}_{12} \hat{R}_{23}\right)=K$ and $\operatorname{tr}_{12}\left(\hat{R}_{12}^{-1} \hat{R}_{23}\right)=Q z I-Q \hat{R}+K$.

(vi) $\operatorname{tr}_{12} \hat{R}=z^{-1} I, \operatorname{tr}_{12} I=I, \operatorname{tr}_{12} K=\mathfrak{s} I$.

(vii) $\operatorname{tr}_{12} X_{+}=Z I$ and $\operatorname{tr}_{12} X_{-}=\left(Z+2 \mathfrak{s} Q^{-1}\right) I$.

Proof. (i) can be verified in a similar way as Lemma 3.2, (i), by direct computations using the formula (6.1) for $\hat{R}$ and the relations $K_{N 1}^{N 1}=q^{-1} z, K_{1 N}^{1 N}=q z^{-1}$. We have $\operatorname{tr}_{2}^{2} T=\operatorname{tr}_{2}^{2} \Phi(\Phi(T))=B\left(\operatorname{tr}_{1}^{1} T\right) C$, hence (i) implies (ii). (iii) and (iv) are immediately obtained from (i) and (7.1). The first formula of (v) can be derived from the known relation $\hat{R}_{12} \hat{R}_{23} K_{12}=K_{23} K_{12}$. Since $\hat{R}^{-1}=\hat{R}-Q I+Q K$, the second formula of (v) follows from the first one combined with $\operatorname{tr}_{12} \hat{R}_{23}=\hat{R}$ and $\left(\operatorname{tr}_{12} K_{12} \hat{R}_{23}\right)_{r s}^{k m}=$ $\delta_{k r}\left(\operatorname{tr}_{1}^{1} \hat{R}\right)_{s}^{m}=(z I)_{r s}^{k m}$ by (i). From $\hat{R} K=z^{-1} K$ we get $\operatorname{tr}_{12} \hat{R}=z^{-1} I$, the rest of (vi) is obvious. (vii) follows at once from (vi).

We introduce six intertwiners $\mathfrak{F}_{ \pm}, \mathfrak{G}_{ \pm}$and $\mathfrak{H}_{ \pm}$of $\operatorname{Mor}(u \otimes u \otimes u)$ by $\mathfrak{F}_{ \pm}:=\alpha_{ \pm}\left(P_{+}\right)_{23} \Phi\left(P_{ \pm}\right)_{12}\left(P_{ \pm}\right)_{23}, \mathfrak{G}_{ \pm}:=\beta_{ \pm}\left(P_{-}\right)_{23} \Phi\left(P_{ \pm}\right)_{12}\left(P_{ \pm}\right)_{23}$ and $\mathfrak{H}_{ \pm}:=\gamma_{ \pm}$ $K_{23} \Phi\left(P_{ \pm}^{ \pm}\right)_{12}$, see Fig. 4 below. Further, we define $\Psi_{ \pm}^{ \pm}(T):=\operatorname{tr}_{1}^{1}\left(T \Phi\left(P_{ \pm}\right)_{12}\right)$ for $T \in \operatorname{Mor}(u \otimes u \otimes u)$. Note that $\Psi_{ \pm}(T) \in \operatorname{Mor}(u \otimes u)$ by Lemma 7.2, (i).

Lemma 7.5. For $\tau \in\{+,-\}$, we have:

(i) $T_{\tau}+K_{12}=\varphi_{\tau}^{+} \mathfrak{F}_{+}+\varphi_{\tau}^{-} \mathfrak{F}_{-}+\psi_{\tau}^{+} \mathfrak{G}_{+}+\psi_{\tau}^{-} \mathfrak{G}_{-}+\mu_{\tau}^{+} \mathfrak{H}_{+}+\mu_{\tau}^{-} \mathfrak{H}_{-}$.

(ii) $\Psi_{ \pm}\left(T_{\tau}+K_{12}\right)=\varphi_{\tau}^{ \pm} P_{+}+\psi_{\tau}^{ \pm} P_{-}+\mu_{\tau}^{ \pm} P_{0}$.

(iii) $\operatorname{tr}_{12}\left(\Psi_{ \pm}\left(T_{\tau}+K_{12}\right)\right)=\mu_{\tau}^{ \pm} I$.

Proof. All assertions can be verified by direct (lengthy) computations inserting the definitions of the constants $\varphi_{\tau}^{ \pm}, \psi_{\tau}^{ \pm}, \mu_{\tau}^{ \pm}$and using the above formulas for $P_{ \pm}$and $\Phi\left(P_{ \pm}\right)$and of Lemma 7.4. We omit the details.

The formulas in Lemma 7.5 give important links between the bicovariant differential calculus $\left(\Gamma_{\tau}, d\right)$ and its associated ad-invariant right ideal $\mathscr{R}_{\tau}$ : The structure of the calculus $\left(\Gamma_{\tau}, d\right)$ is completely described by the linear transformation $T_{\tau}$, while the ideal $\mathscr{R}_{\tau}$ is characterized by the six constants $\varphi_{\tau}^{ \pm}, \psi_{\tau}^{ \pm}, \mu_{\tau}^{ \pm}$as shown by Lemma 8.2 below.

The linear transformations $T_{+}$and $T_{-}$belong to the algebra $\operatorname{Mor}(u \otimes u \otimes u)$. This algebra is isomorphic to the Birman-Wenzl-Murakami algebra (cf. [BW], $[\mathrm{Re}]) \mathscr{C}_{3}(l, m)$ with parameters $l=-i z^{-1}, m=i Q$. An isomorphism of $\mathscr{C}_{3}(l, m)$ onto $\operatorname{Mor}(u \otimes u \otimes u)$ is defined by $G_{j} \rightarrow i \hat{R}_{j, j+1}, E_{j} \rightarrow-K_{j, j+1}$ for $j=1,2$. Note that the vector space dimension of $\operatorname{Mor}(u \otimes u \otimes u)$ is 15 .

Figures 4, 5 and 6 give the graphical representations of some important intertwiners, operations and identities appearing in Part II. 


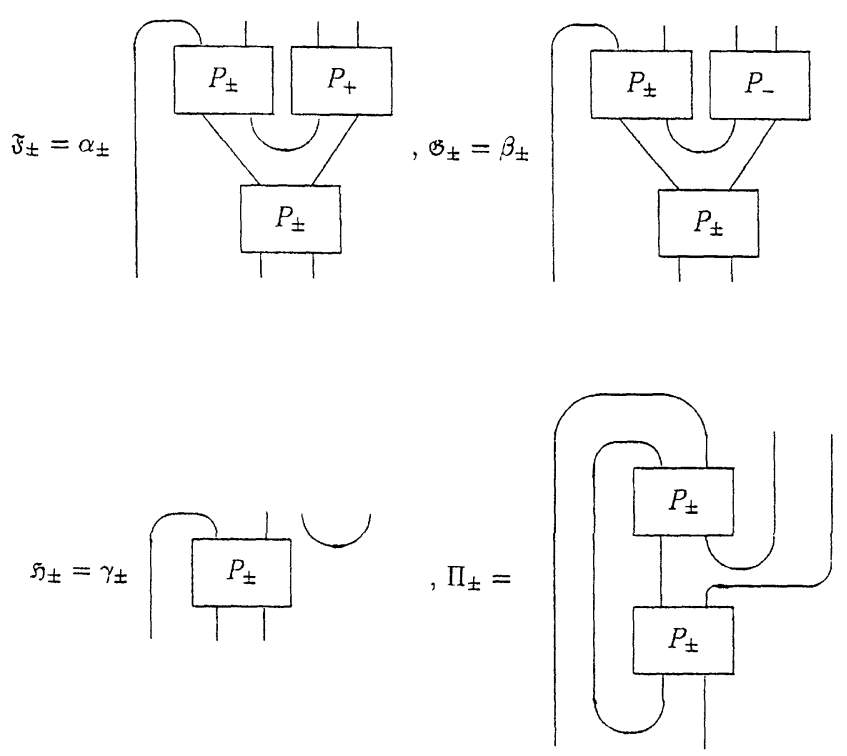

Fig. 4. The graphical representations of some intertwiners for $\mathscr{A}=O_{q}(N)$ and $\mathscr{A}=S p_{q}(N)$

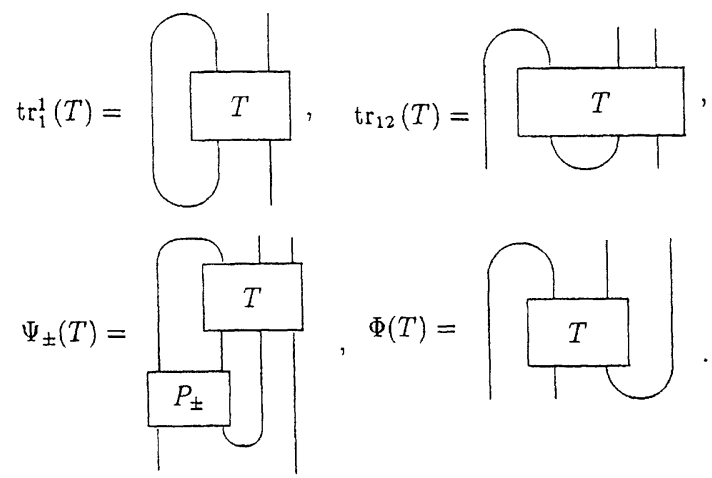

Fig. 5. The graphical representations of some operations with intertwiners for $\mathscr{A}=O_{q}(N)$ and $\mathscr{A}=S p_{q}(N)$

Lemma 7.4, (i):

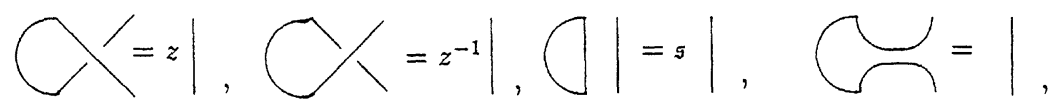

Lemma 7.4, (v):

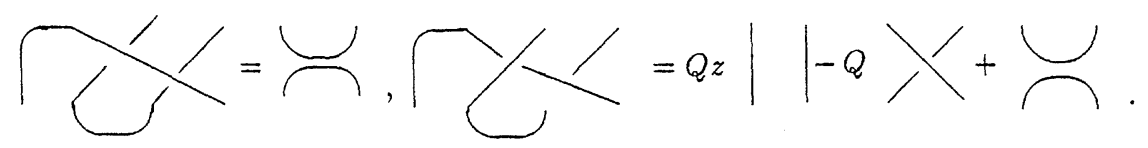

Fig. 6. The graphical representations of some identities of intertwiners for $\mathscr{A}=O_{q}(N)$ and $\mathscr{A}=S p_{q}(N)$ 


\section{Proofs of Theorems 6.1 and 6.2}

Suppose that $\mathscr{R}$ is an ad-invariant right ideal of ker $\varepsilon$ such that $\operatorname{ker} \varepsilon=\mathscr{R} \oplus \tilde{\mathscr{A}}_{1}$. As usual, $a \equiv b$ means that $a-b \in \mathscr{R}$. Our aim is to show that $\mathscr{R}=\mathscr{R}_{+}$or $\mathscr{R}=\mathscr{R}_{-}$.

Lemma 8.1. Suppose that $N \geqq 4$. Then we have:

(i) $\operatorname{ad} \Gamma \mathscr{A}_{1} \cong u^{c} \otimes u \cong u \otimes u \cong[0] \oplus[2] \oplus\left[1^{2}\right]$

The projections of $\mathscr{A}_{1}$ onto the subspaces for the Young patterns [0], [2] and $\left[1^{2}\right]$ are ${ }_{1}^{1} P_{0},{ }_{1}^{1} P_{+}$and ${ }_{1}^{1} P_{-}$, respectively.

(ii) The linear mappings $A_{ \pm}$defined by $A_{ \pm}\left(u_{j}^{i}\right):=\left(v_{ \pm}\right)_{j}^{i}, i, j=1, \ldots, N$, belong to the space $\operatorname{Mor}\left(\operatorname{ad}\left\lceil\mathscr{A}_{1}\right.\right.$, ad $\left\lceil\mathscr{A}_{2}\right)$.

(iii) $\operatorname{ad} \Gamma \mathscr{A}_{2} \cong([2,2] \oplus[3,1] \oplus[4]) \oplus\left([2,2] \oplus[2,1,1] \oplus\left[1^{4}\right]\right) \oplus 2[2] \oplus$ $2\left[1^{2}\right] \oplus 3[0]$.

The mappings $S_{+}$and $S_{-}$are the projections for the two subrepresentations in brackets. The mappings $F_{ \pm}, G_{ \pm}$and $H_{ \pm}$defined by (8.5)-(8.7) below are projections for each two subrepresentations [2], [1 $\left.{ }^{2}\right]$ and [0], respectively. The third subspace of $\mathscr{A}_{2}$ for the trivial representation $[0]$ is $\langle\mathbb{1}\rangle$.

Proof. (i): Decomposing the tensor product represetation $[1] \otimes[1]$ in case of $B_{n}, C_{n}$ and $D_{n}$, we obtain $u \otimes u \cong[0] \oplus[2] \oplus\left[1^{2}\right]$ and the corresponding projections are the three projections $P_{0}, P_{+}, P_{-}$of the intertwining space $\operatorname{Mor}(u \otimes u)$. Since $u^{c}=B^{t} u C^{t}$ by (6.2), we have $u^{c} \otimes u \cong[0] \oplus[2] \oplus\left[1^{2}\right]$ and the corresponding projections are ${ }_{1}^{1} P_{\tau}=B_{1}^{t} P_{\tau} C_{1}^{t}$ for $\tau=0,+,-$. The assertion for ad $\left\lceil\mathscr{A}_{1}\right.$ follows by applying the isomorphism $\mathbf{I}_{1} \in \operatorname{Mor}\left(u^{c} \otimes u, \operatorname{ad}\left\lceil\mathscr{A}_{1}\right)\right.$.

(ii): From $\check{P}_{ \pm} \in \operatorname{Mor}\left(u^{c} \otimes u^{c}\right),(1.2)$ and Lemma 7.1 we obtain

$$
\begin{aligned}
\operatorname{ad}\left(v_{ \pm}\right)_{j}^{i} & =\left(P_{ \pm}\right)_{k m}^{x i} D_{x}^{y} \operatorname{ad}\left(u_{y}^{k} u_{j}^{m}\right) \\
& =\left(\check{P}_{ \pm}\right)_{i x}^{m k} D_{x}^{y} u_{s_{1}}^{r_{1}} u_{s_{2}}^{r_{2}} \otimes\left(u^{c}\right)_{m}^{r_{2}}\left(u^{c}\right)_{k}^{r_{1}} u_{y}^{s_{1}} u_{j}^{s_{2}} \\
& =\left(\check{P}_{ \pm}\right)_{m k}^{r_{2} r_{1}} u_{s_{1}}^{r_{1}} u_{s_{2}}^{r_{2}} \otimes\left(u^{c}\right)_{i}^{m}\left(\left(u^{c}\right)_{x}^{k} u_{y}^{s_{1}} D_{x}^{y}\right) u_{j}^{s_{2}} \\
& =\left(P_{ \pm}\right)_{r_{1} r_{2}}^{k m} D_{k}^{s_{1}} u_{s_{1}}^{r_{1}} u_{s_{2}}^{r_{2}} \otimes\left(u^{c}\right)_{i}^{m} u_{j}^{s_{2}} \\
& =\left(v_{ \pm}\right)_{s_{2}}^{m} \otimes\left(u^{c}\right)_{i}^{m} u_{j}^{s_{2}}
\end{aligned}
$$

so that $A_{ \pm} \in \operatorname{Mor}\left(\operatorname{ad}\left\lceil\mathscr{A}_{1}, \operatorname{ad} \Gamma \mathscr{A}_{2}\right)\right.$ by (1.2).

(iii): First let us note that

$$
\left(u^{c} \otimes u^{c}\right) \otimes(u \otimes u)=\left([0] \oplus[2] \oplus\left[1^{2}\right]\right) \otimes\left([0] \oplus[2] \oplus\left[1^{2}\right]\right) .
$$

The projections for the decomposition of $u^{c} \otimes u^{c}$ are $\check{P}_{0}, \check{P}_{+}, \check{P}_{-} \in \operatorname{Mor}\left(u^{c} \otimes u^{c}\right)$, while the projections for $u \otimes u$ are $P_{0}, P_{+}, P_{-} \in \operatorname{Mor}(u \otimes u)$. The same reasoning as in the case of $S L_{q}(N)$ (see the proof of Lemma 4.1, (iii)) shows that $\operatorname{im}\left(\check{P}_{\tau} \otimes P_{\tau^{\prime}}\right) \subseteq \operatorname{ker} \mathbf{I}_{2}$ for $\tau \neq \tau^{\prime}, \tau, \tau^{\prime} \in\{0,+,-\}$. For $B_{n}$ and $D_{n}$, we have $\operatorname{dimim}\left(\check{P}_{0} \otimes P_{0}+\check{P}_{+} \otimes P_{+}+\check{P}_{-} \otimes P_{-}\right) \leqq 1+\left(\frac{1}{2} N(N+1)-1\right)^{2}+$ $\left(\frac{1}{2}(N(N-1))^{2}=\frac{1}{2}\left(N^{4}-N^{2}-2 N+4\right)=\operatorname{dim} \operatorname{ad}\left[\mathscr{A}_{2}\right.\right.$. Similarly, $\operatorname{dim} \operatorname{im}\left(\check{P}_{0} \otimes P_{0}+\right.$ $\left.\check{P}_{+} \otimes P_{+}+\check{P}_{-} \otimes P_{-}\right)=\frac{1}{2}\left(N^{4}-N^{2}+2 N+4\right)=\operatorname{dim} \operatorname{ad} \otimes P_{-}\left\lceil\mathscr{A}_{2}\right.$ in case of $C_{n}$. Hence $\check{P}_{0} \otimes P_{0}+\check{P}_{+} \otimes P_{+}+\check{P}_{-} \otimes P_{-}=\check{P}_{0} \otimes P_{0} \oplus \check{P}_{+} \otimes P_{+} \oplus \check{P}_{-} \otimes P_{-}$and $\mathbf{I}_{2}\lceil\mathscr{W}$ is an isomorphism of $\mathscr{W}:=\operatorname{im}\left(\check{P}_{0} \otimes P_{0} \oplus \check{P}_{+} \otimes P_{+} \oplus \check{P}_{-} \otimes P_{-}\right)$onto $\mathscr{A}_{2}$. Therefore, using once more the general rules for decomposing tensor product representations 
([BR, H]), it follows from (8.1) that

$$
\begin{aligned}
\operatorname{ad} \Gamma \mathscr{A}_{2} \cong & ([0] \otimes[0]) \oplus([2] \otimes[2]) \oplus\left(\left[1^{2}\right] \otimes\left[1^{2}\right]\right) \\
\cong & ([0]) \\
& \oplus\left([2,2] \oplus[3,1] \oplus[4] \oplus[2] \oplus\left[1^{2}\right] \oplus[0]\right) \\
& \oplus\left([2,2] \oplus[2,1,1] \oplus\left[1^{4}\right] \oplus[2] \oplus\left[1^{2}\right] \oplus[0]\right) .
\end{aligned}
$$

Let $E_{0}, E_{+}$and $E_{-}$denote the projections corresponding to the subrepresentations of $\operatorname{ad}\left\lceil\mathscr{A}_{2}\right.$ in the preceding three lines. By construction, $E_{\tau}\left(u_{j}^{i} u_{m}^{k}\right)=\left(P_{\tau} u_{1} u_{2}\right)_{j m}^{i k}$ for $\tau=0,+,-$ and $i, j, k, m=1, \ldots, N$. From $\left(P_{0}\right)_{r s}^{i k}=\frac{1}{s} C_{k}^{i} B_{s}^{\mathrm{r}}$ and (6.2) we get $E_{0}\left(u_{j}^{i} u_{m}^{k}\right)=\left(P_{0}\right)_{j m}^{i k} \mathbb{1}$. We denote by $S_{+}$and $S_{-}$the projections corresponding to $[2,2] \oplus[3,1] \oplus[4]$ and $[2,2] \oplus[2,1,1] \oplus\left[1^{4}\right]$ in $(8.3)$ and $(8.4)$, respectively. Further, we define linear mappings $F_{ \pm}, G_{ \pm}, H_{ \pm}: \mathscr{A}_{2} \rightarrow \mathscr{A}_{2}$ by

$$
\begin{aligned}
& F_{ \pm}\left(u_{j}^{i} u_{m}^{k}\right):=\alpha_{ \pm}\left(P_{ \pm}\left(f_{ \pm}\right)_{2} P_{ \pm}\right)_{j m}^{i k}, \\
& G_{ \pm}\left(u_{j}^{i} u_{m}^{k}\right):=\beta_{ \pm}\left(P_{ \pm}\left(g_{ \pm}\right)_{2} P_{ \pm}\right)_{j m}^{i k}, \\
& H_{ \pm}\left(u_{j}^{i} u_{m}^{k}\right):=\gamma_{ \pm}\left(P_{ \pm}\right)_{j m}^{i k} V_{ \pm},
\end{aligned}
$$

where the $\alpha_{ \pm}, \beta_{ \pm}, \gamma_{ \pm}$are the constants defined in Sect. 6. It is easily seen that these mappings are well-defined and that $\operatorname{im} F_{ \pm}, \operatorname{im} G_{ \pm}, \operatorname{im} H_{ \pm} \subseteq \operatorname{im} E_{ \pm}$. Applying Lemma 7.3, we get

$$
\begin{aligned}
F_{ \pm}\left(\left(f_{ \pm}\right)_{j}^{i}\right) & =\alpha_{ \pm}\left({ }_{1}^{1} P_{+}\right)_{i j}^{k m}\left(P_{ \pm}\right)_{r s}^{p k} D_{p}^{l}\left(P_{ \pm}\right)_{x y}^{r s}\left(f_{ \pm}\right)_{z}^{y}\left(P_{ \pm}\right)_{l m}^{x z} \\
& =\alpha_{ \pm}\left(P_{+} C_{1}^{t}\right)_{i j}^{r m}\left(B_{k}^{r} D_{p}^{l}\left(P_{ \pm}\right)_{x w}^{p k}\left(P_{ \pm}\right)_{l m}^{x z} C_{s}^{w}\right) B_{y}^{s}\left(f_{ \pm}\right)_{z}^{y} \\
& =\alpha_{ \pm}\left(P_{+} C_{1}^{t}\right)_{i j}^{r m}\left(\Pi_{ \pm}\right)_{r m}^{s z} B_{y}^{s}\left(f_{ \pm}\right)_{z}^{y}=\left({ }_{1}^{1}\left(\Pi_{ \pm} P_{+}\right)\right)_{i j}^{y z}\left(f_{ \pm}\right)_{z}^{y} \\
& =\alpha_{ \pm}\left({ }_{1}^{1}\left(P_{+} \Pi_{ \pm}\right)\right)_{i j}^{y z}\left(f_{ \pm}\right)_{z}^{y} \\
& =\alpha_{ \pm}\left(\frac{1}{\alpha_{ \pm}}{ }_{1}^{1} P_{+}\right)_{i j}^{y z}\left(f_{ \pm}\right)_{z}^{y}=\left(f_{ \pm}\right)_{j}^{i} .
\end{aligned}
$$

Since $\operatorname{im} F_{ \pm} \subseteq\left\langle\left(f_{ \pm}\right)_{j}^{i}\right\rangle$ by definition, this proves that $F_{ \pm}$is a projection of $\mathscr{A}_{2}$ onto $\left\langle\left(f_{ \pm}\right)_{j}^{i}\right\rangle$. The same reasoning shows that $G_{ \pm}$is a projection of $\mathscr{A}_{2}$ onto $\left\langle\left(g_{ \pm}\right)_{j}^{i}\right\rangle$. By Lemma 7.4, (iii),

$$
\begin{aligned}
H_{ \pm}\left(V_{ \pm}\right) & =D_{i}^{j} D_{s}^{r}\left(P_{ \pm}\right)_{k m}^{s j} H_{ \pm}\left(u_{r}^{k} u_{i}^{m}\right) \\
& =\gamma_{ \pm} D_{i}^{j} D_{s}^{r}\left(P_{ \pm}\right)_{r i}^{s j} V_{ \pm}=\gamma_{ \pm}\left(\operatorname{tr}_{1}^{1}\left(\operatorname{tr}_{1}^{1} P_{ \pm}\right)\right) V_{ \pm}=V_{ \pm},
\end{aligned}
$$

so $H_{ \pm}$is a projection of $\mathscr{A}_{2}$ onto $\left\langle V_{ \pm}\right\rangle$.

Next we show that $F_{+}, G_{+}$and $H_{+}$are mutually orthogonal. (Here we call two projections $E$ and $F$ orthogonal if $E F=F E=0$.) We have

$$
\begin{aligned}
F_{+}\left(\left(g_{+}\right)_{j}^{i}\right) & =\left({ }_{1}^{1} P_{-}\right)_{i j}^{k m}\left(P_{+}\right)_{r s}^{p k} D_{p}^{l} F_{+}\left(u_{l}^{r} u_{m}^{s}\right) \\
& =\alpha_{+}\left(P_{-} C_{1}^{t}\right)_{i j}^{r m}\left(B_{k}^{r} D_{p}^{l}\left(P_{+}\right)_{x w}^{p k}\left(P_{+}\right)_{l m}^{x z} C_{s}^{w}\right) B_{y}^{s}\left(f_{+}\right)_{z}^{y} \\
& =\alpha_{+}\left(P_{-} C_{1}^{t}\right)_{i j}^{r m}\left(\Pi_{+}\right)_{r m}^{s z} B_{y}^{s}\left(f_{+}\right)_{z}^{y} \\
& =\alpha_{+}\left({ }_{1}^{1}\left(\Pi_{+} P_{-}\right)\right)_{i j}^{y z}\left(f_{+}\right)_{z}^{y}=\alpha_{+}\left(P_{+} \Pi_{+} P_{-}\right)_{i j}^{r s}\left(v_{+}\right)_{s}^{r}=0,
\end{aligned}
$$


where the last equality follows from $P_{+} P_{-}=0$. Similarly, $G_{+}\left(\left(f_{+}\right)_{j}^{i}\right)=0$, so that $F_{+}$and $G_{+}$are orthogonal. Writing $H_{+}$as $H_{+}\left(u_{j}^{i} u_{m}^{k}\right)=\gamma_{+}\left(P_{+}\left(h_{+}\right)_{2} P_{+}\right)_{j m}^{i k}$ with $\left(h_{+}\right)_{j}^{i}:=\left({ }_{1}^{1} P_{0}\right)_{i j}^{r s}\left(v_{+}\right)_{s}^{r}=\mathfrak{s}^{-1} \delta_{i j} V_{+}$, the same reasoning shows that $F_{+} H_{+}=$ $G_{+} H_{+}=0$. From Lemma 7.4, (iv),

$$
\begin{aligned}
H_{+}\left(\left(f_{+}\right)_{j}^{i}\right) & =\gamma_{+}\left({ }_{1}^{1} P_{+}\right)_{i j}^{k m} D_{s}^{r}\left(P_{+}\right)_{r m}^{s k} V_{+} \\
& =\gamma+\left({ }_{1}^{1} P_{+}\right)_{i j}^{k m}\left(\operatorname{tr}_{1}^{1} P_{+}\right)_{m}^{k} V_{+} \\
& =\mathfrak{s}^{-1}\left({ }_{1}^{1} P_{+}\right)_{i j}^{m m} V_{+}=\mathfrak{s}^{-1}\left(\operatorname{tr}_{1}^{1} \Phi\left(P_{+}\right)\right)_{j}^{i} V_{+}=0,
\end{aligned}
$$

and similarly $H_{+}\left(\left(g_{+}\right)_{j}^{i}\right)=0$, so that $H_{+} F_{+}=H_{+} G_{+}=0$, i.e. $F_{+}$and $H_{+}$, resp. $G_{+}$and $H_{+}$are orthogonal.

By the preceding, $\mathscr{V}_{+}:=\left\langle\left(v_{+}\right)_{j}^{i}\right\rangle$ is the direct sum of the subspaces im $F_{+}, \operatorname{im} G_{+}$ and im $H_{+}$. From (8.1) we see at once that these three spaces are ad-invariant. Hence the mapping $A_{+}$from (ii) is injective and $A_{+}$gives the equivalence of ad $\left\lceil\mathscr{A}_{1}\right.$ and ad $\left\lceil\mathscr{V}_{+}\right.$. Therefore, by (i) and by the definitions of $F_{+}, G_{+}$and $H_{+}$, the latter are the projections corresponding to the Young patterns $[2],\left[1^{2}\right]$ and $[0]$ in (8.3) and $S_{+}=E_{+}-F_{+}-G_{+}-H_{+}$has the form given in Sect. 6. A similar reasoning works for $F_{-}, G_{-}, H_{-}$and $S_{-}$.

Lemma 8.2. There exist complex numbers $\varphi^{+}, \varphi^{-}, \psi^{+}, \psi^{-}, \mu^{+}$and $\mu^{-}$such that $\mathscr{R}_{12}=\left\langle\tilde{V}_{+}-\mu^{+} \tilde{U}\right\rangle \oplus\left\langle\tilde{V}_{-}-\mu^{-} \tilde{U}\right\rangle \oplus \tilde{\mathscr{F}}_{+} \oplus \mathscr{F}_{-} \oplus \mathscr{G}_{+} \oplus \mathscr{G}_{-} \oplus \operatorname{im} S_{+} \oplus \operatorname{im} S_{-}$,

where $\mathscr{F}_{ \pm}:=\left\langle\left(f_{ \pm}\right)_{j}^{i}-\varphi^{ \pm}\left(u_{+}\right)_{j}^{i}: i, j=1, \ldots, N\right\rangle$ and $\mathscr{G}_{ \pm}:=\left\langle\left(g_{ \pm}\right)_{j}^{i}-\psi^{ \pm}\left(u_{-}\right)_{j}^{i}\right.$ : $i, j=1, \ldots, N\rangle$.

Proof. First let us assume that $N \geqq 4$. We take an ad-invariant linear subspace $\mathscr{F}$ of $\tilde{\mathscr{A}}_{1} \oplus \tilde{\mathscr{I}}_{2}$ such that $\mathscr{R}_{12} \oplus \mathscr{\mathscr { F }}=\tilde{\mathscr{I}}_{1} \oplus \tilde{\mathscr{I}}_{2}$. Since ker $\varepsilon=\mathscr{R} \oplus \tilde{\mathscr{I}}_{1}$ by assumption, $\mathscr{R}_{12} \oplus \tilde{\mathscr{A}}_{1}=\tilde{\mathscr{A}}_{1} \oplus \tilde{\mathscr{A}}_{2}$ and hence $\operatorname{ad}\left[\mathscr{F} \cong \mathrm{ad}\left[\mathscr{A}_{1} \cong[0] \oplus[2] \oplus\left[1^{2}\right]\right.\right.$ by Lemma 8.1 , (i). Since $\mathbb{1} \in \mathscr{A}_{2}$ and $\mathbb{1} \notin \mathscr{A}_{1}$, we have $\operatorname{ad}\left\lceil\left(\mathscr{A}_{1} \oplus \mathscr{A}_{2}\right)=\right.$ $\operatorname{ad}\left\lceil\left(\tilde{\mathscr{A}}_{1} \oplus \tilde{\mathscr{A}}_{2}\right) \oplus[0]\right.$, where the subspace for the trivial representation $[0]$ is $\langle\mathbb{1}\rangle$. Therefore, from the decompositions of ad $\left\lceil\mathscr{A}_{1}, \operatorname{ad}\left\lceil\mathscr{A}_{2}\right.\right.$ and ad $\lceil\mathscr{F}$ it follows that

$$
\operatorname{ad}\left\lceil\mathscr{R}_{12} \cong([2,2] \oplus[3,1] \oplus[4]) \oplus\left([2,2], \oplus[2,1,1] \oplus\left[1^{4}\right]\right)\right.
$$

$$
\oplus 2[2] \oplus 2\left[1^{2}\right] \oplus 2[0] \text {. }
$$

By Lemma 8.1,(iii), the subspaces for subrepresentations in brackets are im $S_{+}$and $\operatorname{im} S_{-}$. The two subspaces for [2] are ad-invariant subspaces of $\left\langle\left(f_{+}\right)_{j}^{i}\right\rangle \oplus$ $\left\langle\left(f_{-}\right)_{j}^{i}\right\rangle \oplus\left\langle\left(u_{+}\right)_{j}^{i}\right\rangle$. Since $\left\langle\left(u_{+}\right)_{j}^{i}\right\rangle \cap \mathscr{R}=\{0\}$ by the assumption $\operatorname{ker} \varepsilon=\mathscr{R} \oplus \tilde{\mathscr{A}}_{1}$, we conclude that there exist numbers $\varphi^{+}, \varphi^{-} \in \mathbb{C}$ such that the subspaces for [2] are $\mathscr{F}_{+}$and $\mathscr{F}_{-}$. Similarly, the subspaces for $\left[1^{2}\right]$ and $[0]$ can be written as $\mathscr{G}_{+}, \mathscr{G}_{-}$ and $\left\langle\tilde{V}_{+}-\mu^{+} \tilde{U}\right\rangle,\left\langle\tilde{V}_{-}-\mu^{-} \tilde{U}\right\rangle$, respectively. This completes the proof of Lemma 8.2 in case $N \geqq 4$.

Suppose now that $N=3$. Clearly, the assertions (i) and (ii) of Lemma 8.1 remain valid. For $N=3$, the Young patterns $[2,2],[2,1,1]$ and $\left[1^{4}\right]$ do not occur in the decompositions of ad $\left\lceil\mathscr{A}_{2}\right.$ in Lemma 8.1, (iii), and of ad $\left\lceil\mathscr{R}_{12}\right.$, cf. [H]. Thus we obtain ad $\left\lceil\mathscr{R}_{12} \cong([3,1] \oplus[4]) \oplus 2[2] \oplus 2\left[1^{2}\right] \oplus 2[0]\right.$ and the corresponding subspaces are $i m S_{+}, \mathscr{F}_{+}, \mathscr{G}_{+}$and $\left\langle\tilde{V}_{+}-\mu^{ \pm} \tilde{U}\right\rangle$ similarly as in case $N \geqq 4$. Since $S_{-} \equiv 0$ for $N=3$, the assertion of Lemma 8.2 is also true if $N=3$. 
Our aim is to determine the numbers $\varphi^{ \pm}, \psi^{ \pm}$and $\mu^{ \pm}$. For these computations we shall use the abbreviations

$$
\begin{aligned}
& a_{ \pm}:=\alpha_{ \pm} \varphi^{ \pm}, b_{ \pm}:=\beta_{ \pm} \psi^{ \pm}, m_{ \pm}:=\gamma_{ \pm} \mu^{ \pm}, \\
& v:=(z-q) Q_{+}^{-1}(q z-1)^{-1}=Q_{+}^{-1}\left(q^{-1}+q_{+}\right), \\
& a^{ \pm}:=q^{ \pm 1} a_{+}-q^{\mp 1} a_{-}, b^{ \pm}:=q^{ \pm 1} b_{+}-q^{\mp 1} b_{-}, \\
& c_{+}:=\left(Q_{+} q_{+}+1\right) a_{+}-q^{-2} a_{-}-b_{+}+\left(q^{-2}-Q_{+} q_{-}\right) b_{-}, \\
& c_{-}:=\left(Q_{+} q_{+}+1+q^{-2}-q^{2}\right) a_{+}-q^{2} a_{-}-b_{+}+\left(q^{-2}-Q_{+} q_{-}\right) b_{-}, \\
& d_{+}:=\left(Q_{+} q_{+} q^{-1}+q^{-3}+q^{-1}-q\right) a_{+}-q a_{-}+q b_{+}+\left(q Q_{+} q_{-}-q^{-1}\right) b_{-}, \\
& d_{-}:=\left(Q_{+} q_{+} q+q\right) a_{+}-q^{-1} a_{-}+q^{-1} b_{+}+\left(q^{-1} Q_{+} q_{-}-q^{-3}\right) b_{-} .
\end{aligned}
$$

Throughout Lemma 8.3 and its proof we shall not sum over repeated indices.

\section{Lemma 8.3.}

(i) $u_{j}^{i} u_{m}^{k} \equiv 0$ if $i \neq j, i \neq m, k \neq j, k \neq m, i \neq k^{\prime}$ and $j \neq m^{\prime}$.

(ii) $u_{j}^{i} u_{k}^{k} \equiv Q_{+}^{-2}\left(\left(a_{+}+a_{-}\right)\left(u_{+}\right)_{j}^{i}+\left(b_{+}+b_{-}\right)\left(u_{-}\right)_{j}^{i}\right)$ if $i \neq j, i \neq k, i \neq k^{\prime}, j \neq k$ and $j \neq k^{\prime}$.

(iii) $u_{j}^{i} u_{i}^{i} \equiv\left(u_{j}^{i} u_{j}^{j} \equiv\right) Q_{+}^{-1}\left(a_{+}\left(u_{+}\right)_{j}^{i}+b_{+}\left(u_{-}\right)_{j}^{i}\right)$ if $i \neq j, i \neq j^{\prime}$ and $i \neq i^{\prime}\left(j \neq j^{\prime}\right)$.

$u_{i^{\prime}}^{i} u_{i^{\prime}}^{i^{\prime}} \equiv u_{i^{\prime}}^{i} u_{i}^{i} \equiv v a_{+} u_{i^{\prime}}^{i}$ if $i \neq i^{\prime}$.

$u_{i}^{i} u_{i^{\prime}}^{i} \equiv Q_{+}^{-1}\left(q+D_{i^{\prime}}^{i^{\prime}}\left(q_{+}-Q\right)\right) a_{+} u_{i^{\prime}}^{i}$ if $i<i^{\prime}$.

$u_{i^{\prime}}^{i^{\prime}} u_{i^{\prime}}^{i} \equiv Q_{+}^{-1}\left(q^{-1}+D_{i}^{i} q_{+}\right) a_{+} u_{i^{\prime}}^{i}$ if $i<i^{\prime}$.

(iv) $u_{j}^{i} u_{j k}^{j} \equiv\left(u_{k}^{j} u_{j}^{i} \equiv\right) Q_{+}^{-2}\left(a^{+}\left(u_{+}\right)_{k}^{i}+b^{+}\left(u_{-}\right)_{k}^{i}\right)$ if $i \neq j, i \neq j^{\prime}, i \neq k, j \neq k^{\prime}$ and $j<k(j<i)$.

$u_{j}^{i} u_{j k}^{j} \equiv\left(u_{k}^{j} u_{j}^{i} \equiv\right) Q_{+}^{-2}\left(a^{-}\left(u_{+}\right)_{k}^{i}+b^{-}\left(u_{-}\right)_{k}^{i}\right)$ if $i \neq j, \quad i \neq j^{\prime}, i \neq k, j \neq k^{\prime}$ and $j>k(j>i)$.

(v) $u_{i^{\prime}}^{i} u_{j}^{i^{\prime}} \equiv\left(u_{j}^{j^{\prime}} u_{j^{\prime}}^{i} \equiv\right) Q_{+}^{-3}\left(d_{+} u_{j}^{i}+K_{j j^{\prime}}^{i i^{\prime}} c_{-} u_{i^{\prime}}^{j^{\prime}}\right)$ if $i \neq j, j<i^{\prime}$ and $i \neq i^{\prime}\left(j \neq j^{\prime}\right)$.

$u_{i^{\prime}}^{i} u_{j}^{i^{\prime}} \equiv\left(u_{j}^{j^{\prime}} u_{j^{\prime}}^{i} \equiv\right) Q_{+}^{-3}\left(d_{-} u_{j}^{i}+K_{j j^{\prime}}^{i i^{\prime}} c_{+} u_{i^{\prime}}^{j^{\prime}}\right)$ if $i \neq j, j>i^{\prime}$ and $i \neq i^{\prime}\left(j \neq j^{\prime}\right)$.

(vi) $u_{i}^{j} u_{i}^{j^{\prime}} \equiv Q_{+}^{-1}\left(q_{+}-\theta\left(j^{\prime}-i\right) Q\right) a_{+} K_{j j^{\prime}}^{i i^{\prime}} u_{i}^{i^{\prime}}$ if $i \neq j, i \neq j^{\prime}$ and $i \neq i^{\prime}$.

$u_{j}^{i} u_{j^{\prime}}^{i} \equiv Q_{+}^{-1}\left(q_{+}-\theta\left(j^{\prime}-i\right) Q\right) a_{+} K_{j j^{\prime}}^{i i^{\prime}} u_{i^{\prime}}^{i}$ if $i \neq j, i \neq j^{\prime}$ and $i \neq i^{\prime}$.

(vii) $u_{i}^{i} u_{i}^{i} \equiv a_{+}\left(u_{+}\right)_{i}^{i}+b_{+}\left(u_{-}\right)_{i}^{i}+m_{+} \tilde{U}+\mathbb{1}$ if $i \neq i^{\prime}$.

(viii) $u_{j}^{i} u_{i}^{j} \equiv Q_{+}^{-2}\left(a^{+}\left(u_{+}\right)_{j}^{j}+a^{-}\left(u_{+}\right)_{i}^{i}+b^{+}\left(u_{-}\right)_{j}^{j}+b^{-}\left(u_{-}\right)_{i}^{i}\right)+Q_{+}^{-1}\left(m_{+}-m_{-}\right) \tilde{U}$ if $i<j$ and $i \neq j^{\prime}$.

Proof. By Lemma 8.2, we have im $S_{ \pm} \subseteq \mathscr{R}$. Since $u_{j}^{i} u_{m}^{k}=\sum_{\mathrm{r}, s}\left(P_{+}\right)_{\mathrm{r} s}^{i k} u_{j}^{\mathrm{r}} u_{m}^{s}+$ $\sum_{\mathrm{r}, s}\left(P_{-}\right)_{\mathrm{r} s}^{i k} u_{j}^{\mathrm{r}} u_{m}^{s}+\left(P_{0}\right)_{j m}^{i k} \mathbb{1}$, by the definitions of $S_{ \pm}$this implies that

$$
u_{j}^{i} u_{m}^{k}-\delta_{i j} \delta_{k m} \mathbb{1} \equiv\left(F_{+}+F_{-}+G_{+}+G_{-}+H_{+}+H_{-}\right)\left(u_{j}^{i} u_{m}^{k}\right)^{\sim} .
$$


All assertions of Lemma 8.3 are derived from (8.9) by direct computations. For this we essentially use the explicit formulas (6.1) and (7.1) for $\hat{R}$ and $P_{ \pm}$, respectively. As a sample, we carry out the proof of (v.1).

From (6.1) and (7.1) it follows that a term $\left(P_{ \pm}\right)_{k m}^{i i^{\prime}}\left(P_{ \pm}\right)_{i^{\prime} j}^{k r}$ is non-zero only in the two cases $k=i^{\prime}, m=i, r=j$ and $k=j, m=j^{\prime}, r=i^{\prime}$. Since $i \neq i^{\prime}$ and $i^{\prime}>j$, we have $\left(P_{ \pm} i_{i^{\prime} i}^{i i^{\prime}}\left(P_{ \pm}\right)_{i^{\prime} j}^{i^{\prime} j}=Q_{+}^{-1}\left( \pm q^{-1}+q_{ \pm}\right) Q_{+}^{-1} q^{\mp 1}\right.$ in the first case and $\left(P_{ \pm}\right)_{j j^{\prime}} i^{\prime}\left(P_{ \pm}\right)_{i^{\prime} j}^{j i^{\prime}}=$ $Q_{+}^{-1} K_{j j^{\prime}}^{i i^{\prime}}\left(q_{ \pm} \mp Q\right) Q_{+}^{-1}( \pm 1)$ in the second case. Since $\left(f_{ \pm}\right)_{r}^{m} \equiv \varphi^{ \pm}\left(u_{+}^{ \pm}\right)_{r}^{m}$, this gives

$$
\begin{aligned}
F_{ \pm}\left(u_{i^{\prime}}^{i} u_{j}^{i^{\prime}}\right) & =\sum_{k, m, r} \alpha_{ \pm}\left(P_{ \pm}\right)_{k m}^{i i^{\prime}}\left(f_{ \pm}\right)_{r}^{m}\left(P_{ \pm}\right)_{i^{\prime} j}^{k r} \\
& \equiv \alpha_{ \pm} \varphi^{ \pm} Q_{+}^{-2}\left[\left( \pm q^{-1}+q_{ \pm}\right) q^{\mp 1}\left(u_{+}\right)_{j}^{i}+\left(q_{ \pm} \mp Q\right)( \pm 1) K_{j j^{\prime}}^{i i^{\prime}}\left(u_{+}\right)_{i^{\prime}}^{j^{\prime}}\right],
\end{aligned}
$$

so that

$$
\begin{aligned}
\left(F_{+}+F_{-}\right)\left(u_{i^{\prime}}^{i} u_{j}^{i^{\prime}}\right) \equiv & Q_{+}^{-2}\left(\left(a_{+}\left(q^{-1}+q_{+}\right) q^{-1}+a_{-}\left(-q^{-1}+q_{-}\right) q\right)\left(u_{+}\right)_{j}^{i}\right. \\
& \left.+\left(a_{+}\left(q_{+}-Q\right)-a_{-}\left(q_{-}+Q\right)\right) K_{j j^{\prime}}^{i i^{\prime}}\left(u_{+}\right) j_{i^{\prime}}^{j^{\prime}}\right) .
\end{aligned}
$$

Similarly, we obtain

$$
\begin{aligned}
\left(G_{+}+G_{-}\right)\left(u_{i^{\prime}}^{i} u_{j}^{i^{\prime}}\right) \equiv & Q_{+}^{-2}\left(\left(b_{+}\left(q^{-1}+q_{+}\right) q^{-1}+b_{-}\left(-q^{-1}+q_{-}\right) q\right)\left(u_{-}\right)_{j}^{i}\right. \\
& \left.+\left(b_{+}\left(q_{+}-Q\right)-b_{-}\left(q_{-}+Q\right)\right) K_{j j^{\prime}}^{i i^{\prime}}\left(u_{-}\right)_{i^{\prime}}^{j^{\prime}}\right) .
\end{aligned}
$$

Moreover, we have $H_{ \pm}\left(u_{i^{\prime}}^{i} u_{j}^{i}\right)=\gamma_{ \pm}\left(P_{ \pm}\right)_{i^{\prime} j}^{i i^{\prime}} V_{ \pm}=0$ and $\left(u_{ \pm}\right)_{m}^{k}=$ $Q_{+}^{-1}\left(q^{\mp 1} u_{m}^{k} \pm K_{m m^{\prime}}^{k k^{\prime}} u_{k^{\prime}}^{m^{\prime}}\right)$ if $k^{\prime}>m$ and $k \neq m$. Combining these formulas with (8.9) and using the definitions of $d_{+}$and $c_{-}$, (v.1) follows.

\section{Lemma 8.4.}

(i) $Q_{+} q_{+} a_{+} d_{-}+a^{+} c_{+}=Q_{+}^{5}-Q_{+}\left(a_{+}+a_{-}\right)^{2}$.

(ii) $Q_{+}\left(q_{+}-Q\right) a_{+} d_{+}+a^{-} c_{-}=Q_{+}^{5}-Q_{+}\left(a_{+}+a_{-}\right)^{2}$.

(iii) $q^{2} Q_{+}^{2} v a_{+} d_{-}=q\left(q^{-1} a_{+}+q b_{+}\right) d_{-}+z^{-1} c_{-}\left(a_{+}-b_{+}\right)$.

(iv) $q^{2} Q_{+}^{2} v a_{+} c_{+}=q\left(q^{-1} a_{+}+q b_{+}\right) c_{+}+z^{-1} d_{+}\left(a_{+}-b_{+}\right)$.

(v) $q^{-2} Q_{+}^{2} v a_{+} d_{+}=q^{-1}\left(q a_{+}+q^{-1} b_{+}\right) d_{+}+z c_{+}\left(a_{+}-b_{+}\right)$.

(vi) $q^{-2} Q_{+}^{2} v a_{+} c_{-}=q^{-1}\left(q a_{+}+q^{-1} b_{+}\right) c_{-}+z d_{-}\left(a_{+}-b_{+}\right)$.

Proof. We carry out the proofs of (ii), (iii) and (iv). Since $C_{s}^{r}=0$ if $s \neq r^{\prime},(6.1)$ and Lemma 8.3, (i), imply that

$$
C_{2^{\prime}}^{2} u_{1^{\prime}}^{1}=\sum_{k} u_{1^{\prime}}^{1} C_{k^{\prime}}^{k} u_{2}^{k} u_{2^{\prime}}^{k^{\prime}} \equiv C_{2^{\prime}}^{2} u_{1^{\prime}}^{1} u_{2}^{2} u_{2^{\prime}}^{2^{\prime}}+C_{1}^{1^{\prime}} u_{1^{\prime}}^{1} u_{2}^{1^{\prime}} u_{2^{\prime}}^{1} \text {. }
$$

Note that (8.10) is also valid for $N=3$. Applying Lemma 8.3, (ii), twice (note that $j=i^{\prime}$ and $k=k^{\prime}$ are not excluded therein) and using that $\left(u_{+}\right)_{j^{\prime}}^{j}=u_{j^{\prime}}^{j}$ and $\left(u_{-}\right)_{j^{\prime}}^{j}=0$, we get $u_{1}^{1} \cdot u_{2}^{2} u_{2^{\prime}}^{2^{\prime}} \equiv Q_{+}^{-4}\left(a_{+}+a_{-}\right)^{2} u_{1^{1}}^{1}$. Similarly, from Lemma 8.3 , (v.1), (vi.2) and (iv.2), we have

$$
\begin{aligned}
u_{1^{\prime}}^{1} u_{2}^{1^{\prime}} u_{2^{\prime}}^{1} & \equiv Q_{+}^{-3}\left(d_{+} u_{2}^{1}+K_{22^{\prime}}^{11^{\prime}} c_{-} u_{1^{\prime}}^{2^{\prime}}\right) u_{2^{\prime}}^{1} \\
& \equiv Q_{+}^{-4}\left(q_{+}-Q\right) d_{+} a_{+} K_{22^{\prime}}^{11^{\prime}} u_{1^{\prime}}^{1}+Q_{+}^{-5} c_{-} a^{-} K_{22^{\prime}}^{11^{\prime}} u_{1^{\prime}}^{1} .
\end{aligned}
$$

Recall that $C_{1}^{11^{\prime}} K_{22^{\prime}}^{11^{\prime}}=C_{2^{\prime}}^{2}$. Putting these facts into (8.10) and using that $C_{2^{\prime}}^{2} \neq 0$ and $u_{1}^{1} \notin \mathscr{R}$ by assumption, we obtain equation (ii). The proof of (i) is quite similar; one replaces $C_{2}^{2}, u_{1}^{1}$, in $(8.10)$ by $C_{2}^{2}, u_{1}^{1}$. 
Due to Lemma 8.3, (iii.2) and (v.2), we have

$$
u_{1}^{1} u_{1}^{1^{\prime}} u_{2}^{1} \equiv q^{2} v a_{+} u_{1}^{1^{\prime}} u_{2}^{1} \equiv q^{2} Q_{+}^{-3} v a_{+}\left(d_{-} u_{2}^{1^{\prime}}+K_{22^{\prime}}^{1^{\prime} c_{+}} u_{1}^{2^{\prime}}\right)
$$

Lemma 8.3, (iii.1) yields $u_{1}^{1} u_{2}^{1} \equiv q Q_{+}^{-2}\left(\left(q^{-1} a_{+}+q b_{+}\right) u_{2}^{1}+K_{22^{\prime}}^{11^{\prime}}\left(a_{+}-b_{+}\right) u_{1^{\prime}}^{2^{\prime}}\right)$. Using the facts that $u_{2}^{1} u_{1}^{1^{\prime}}=q u_{1}^{1^{\prime}} u_{2}^{1}$ and $u_{1}^{2^{\prime}} u_{1}^{1^{\prime}}=q^{-1} u_{1}^{1^{\prime}} u_{1^{\prime}}^{2^{\prime}}$ together with the previous equations and Lemma 8.3, (v.2) and (v.1), we obtain

$$
\begin{aligned}
u_{1}^{1} u_{2}^{1} u_{1}^{1^{\prime}} \equiv & q Q_{+}^{-5}\left\{\left(\left(q^{-1} a_{+}+q b_{+}\right) q d_{-}+K_{22^{\prime}}^{11^{\prime}}\left(a_{+}-b_{+}\right) q^{-1} K_{2^{\prime} 2}^{11^{\prime}} c_{-}\right) u_{2}^{1^{\prime}}\right. \\
& \left.+\left(\left(q^{-1} a_{+}+q b_{+}\right) q K_{22^{\prime}}^{1^{\prime} 1} c_{+}+K_{22^{\prime}}^{11^{\prime}}\left(a_{+}-b_{+}\right) q^{-1} d_{+}\right) u_{1}^{2^{\prime}}\right\}
\end{aligned}
$$

Note that $K_{22^{\prime}}^{11^{\prime}} K_{2^{\prime} 2}^{11^{\prime}}=K_{11^{\prime}}^{11^{\prime}}=D_{1^{\prime}}^{1^{\prime}}=q z^{-1}$. Setting (8.11) and (8.12) into the equation $u_{1}^{1} u_{1}^{1^{\prime}} u_{2}^{1^{\prime}}=q^{-1} u_{1}^{1} u_{2}^{1} u_{1}^{1^{\prime}}$ and comparing the coefficients of $u_{2}^{1^{\prime}}$ and of $u_{1}^{2^{\prime}}$ (both are linearly independent over $\mathscr{R}$ by assumption!), we get (iii) and (iv), respectively. In order to derive (v) and (vi) we begin with the equation $u_{1^{\prime}}^{1^{\prime}} u_{1^{\prime}}^{1^{\prime}} u_{2}^{1^{\prime}}=q u_{1^{\prime}}^{1^{\prime}} u_{2}^{1^{\prime}} u_{1^{\prime}}^{1^{\prime}}$ and argue in a similar way.

The equations (i)-(vi) of Lemma 8.4 form a system of quadratic equations for the numbers $\varphi^{ \pm}$and $\psi^{ \pm}$. Now we solve this system. For this we essentially use our standing assumption that $q$ is not a root of unity. Throughout this derivation, we abbreviate $b:=b_{+}+\left(Q_{+} q_{-}-q^{-2}\right) b_{-}$.

First we check that $a_{+} \neq 0$. Assume to the contrary that $a_{+}=0$. Then we have $c_{+}=-q^{-2} a_{-}-b$ and $c_{-}=-q^{2} a_{-}-b$ by definition and $-q^{-1} a_{-} c_{+}=-q a_{-} c_{-}=Q_{+}^{5}-Q_{+} a_{-}^{2}$ from (i) and (ii). Since $Q_{+} \neq 0, a_{-} \neq 0$. Hence $c_{+}=q^{2} c_{-}$which gives $b=-\left(q^{2}+1+q^{-2}\right) a_{-}$and so $c_{-}=q^{-1} Q_{+} a_{-}$. Inserting the latter into $-q a_{-} c_{-}=Q_{+}^{5}-Q_{+} a_{-}^{2}$ we get $Q_{+}=0$ which is a contradiction.

Further, we show that $d_{-} \neq 0$ or $c_{-} \neq 0$. Assume to the contrary that $d_{-}=c_{-}=0$. Then we have $c_{+}=-q^{-1} Q_{+} b, d_{+}=Q_{+} b, a_{-}=a_{+}\left(Q_{+} q_{+}+q^{-2}\right)$ and $a_{+}+a_{-}=-q^{-1} Q^{-1} b$. Since $a_{+} \neq 0$, the two latter equations imply that $b \neq 0$. Setting the preceding expressions for $c_{+}$and $d_{+}$into (i) and (ii) and using that $b \neq 0$, we find $a_{-}=\left(Q_{+} q^{3} q_{+} z+q^{2}\right) a_{+}$. Compared with $a_{-}=$ $a_{+}\left(Q_{+} q_{+}+q^{-2}\right)$ and $a_{+} \neq 0$, this leads to a contradiction.

If $d_{-}=0$ and $c_{-} \neq 0$, then $a_{+}=b_{+}$by (iii) and hence $\left(q^{-2} Q_{+}^{2} v-1-q^{-2}\right) a_{+}=0$ by (vi) which is a contradiction, because $a_{+} \neq 0$ and $q$ is not a root of unity. Putting the preceding together, we have proved that $d_{-} \neq 0$. By a similar reasoning it follows that $c_{+} \neq 0$.

Next we prove that $c_{-}\left(a_{+}-b_{+}\right) \neq 0$. Assume the contrary. If $c_{-}=0$, then (vi) yields $a_{+}=b_{+}$, since $d_{-} \neq 0$. Hence $a_{+}=b_{+}$. Inserting $a_{+}=b_{+}$into (iii) and using that $d_{-} \neq 0$ and $a_{+} \neq 0$, we obtain a contradiction.

Let $x:=c_{-} d_{-}^{-1}$ and $y:=Q Q_{+} a_{+}\left(a_{+}-b_{+}\right)^{-1}$. Dividing (iv) by $c_{+}$and (iii) by $d_{-}$, we conclude that $x=d_{+} c_{+}^{-1}$. Therefore, by (iii) and (v), $x=q^{2} z Q^{-1}\left(y q_{+}+Q\right)$ and $x^{-1}=q^{-2} z^{-1} Q^{-1}(q q+z y+Q)$. Multiplying both equations, we get a quadratic equation for $y$ which has the solutions $y=0$ and $y=(q z-1)\left(1+q^{-1} z^{-1}\right)$. Since $a_{+} \neq 0, y \neq 0$. The second solution for $y$ yields $x=-q$. Hence $d_{+}+q c_{+}=0$, so that $a_{-}=\left(q^{-1} z-q^{2}\right)(q z-1)^{-1} a_{+}, a^{+}=-Q_{+} q_{+} z a_{+}$and $a^{-}=-Q_{+} q q_{+} a_{+}$by the definitions of $d_{+}$and $c_{+}$. Setting these formulas into (i) and (ii) and using that $c_{-}+q d_{-}=0$, we finally get $\left(a_{+}+a_{-}\right)^{2}=Q_{+}^{4}$. 
First we suppose that $a_{+}+a_{-}=Q_{+}^{2}$. Inserting the above expressions for $a_{-}$and $y$, we obtain $a_{+}=q Q_{+}\left(z-q^{-1}\right)(z-q)^{-1}, a_{-}=q^{-1} Q_{+}\left(z-q^{3}\right)(z-q)^{-1}$ and $b_{+}=Q_{+}\left(q z+q^{-2}\right)\left(z+q^{-1}\right)^{-1}$ so that $\varphi^{ \pm}=\varphi_{+}^{ \pm}$and $\psi^{+}=\psi_{+}^{+}$. From (i) and $c_{-}+q d_{-}=0$ it follows that $b=Q Q_{+}^{2}\left(q^{-1} z+1\right)\left(z+q^{-1}\right)^{-1}$ and $b_{-}=Q_{+}\left(q^{-1} z+1\right)\left(z+q^{-1}\right)^{-1}$, so that $\psi^{-}=\psi_{+}^{-}$. Obviously, if $\left(\varphi^{+}, \varphi^{-}, \psi^{+}, \psi^{-}\right)$ is a solution of (i)-(vi), so is $\left(-\varphi^{+},-\varphi^{-},-\psi^{+},-\psi^{-}\right)$. Therefore, the second possible case $a_{+}+a_{-}=-Q_{+}^{2}$ gives $\varphi^{ \pm}=-\varphi_{+}^{ \pm}=\varphi_{-}^{ \pm} \quad$ and $\psi^{ \pm}=-\psi_{+}^{ \pm}=\psi^{ \pm}$.

Next we compute the numbers $\mu^{+}$and $\mu^{-}$. For this we need the following

Lemma 8.5. (i) $\left(1+q q_{+}\right)\left(a_{+}+q^{2} b_{+}\right) a_{+}+q^{-1}\left(q_{+}+z^{-1}\right)\left(a_{+}-b_{+}\right) a_{+}+$ $Q_{+}\left(q_{+} a_{+}+q_{-} b_{+}\right)\left(\varphi^{+}+\varphi^{-}\right)+Q_{+}^{2}\left(1+m_{+}\left(\varphi^{+}+\varphi^{-}-\mathfrak{s}\right)\right)=q^{4}\left(q^{-1}+q_{+}\right)^{2} a_{+}^{2}$. (ii) $Q_{+}^{2} q^{2} v a_{+}\left(q^{-1} a^{-}+q b^{-}\right)+Q_{+}^{2} q^{-2} v z^{-1} a_{+}\left(a^{-}-b^{-}-q Q a^{+}+q Q b^{+}\right)+$ $\left(a_{+}+a_{-}\right)\left(q^{-1} a^{+}+q b^{+}\right)+q^{2} z^{-1}\left(a^{+}-b^{+}\right)\left(a_{+}+a_{-}\right)+Q_{+}^{2}\left(q_{+}\left(a^{+}+a^{-}\right)+\right.$ $\left.q_{-}\left(b^{+}+b^{-}\right)\right)\left(\varphi^{+}+\varphi^{-}\right)+Q_{+}^{4}\left(m_{+}-m_{-}\right)\left(\varphi^{+}+\varphi^{-}-\mathfrak{s}\right)=0$.

Proof. To prove (i), we use the relation $u_{1}^{1} u_{1}^{1} u_{1^{\prime}}^{1}=q^{4} u_{1}^{1} u_{1}^{1} u_{1}^{1}$. By definition, we have $\left(u_{ \pm}\right)_{1}^{1}=Q_{+}^{-1}\left(q^{\mp 1} u_{1}^{1} \pm z^{-1} u_{1}^{1^{\prime}}+q_{ \pm} U\right), \quad D_{1}^{1}=q^{-1} z, \quad D_{1^{\prime}}^{1^{\prime}}=q z^{-1} \quad$ and $U u_{1^{\prime}}^{1}=\left(v_{+}+v_{-}\right)_{1^{\prime}}^{1}=\left(f_{+}+g_{+}+f_{-}+g_{-}\right)_{1^{\prime}}^{1}=\left(f_{+}+f_{-}\right)_{1^{\prime}}^{1} \equiv\left(\varphi^{+}+\varphi^{-}\right) u_{1^{\prime}}^{1}$. Using these facts and applying Lemma 8.3, (vii), (iii.3), (iii.4), we get

$$
\begin{aligned}
u_{1}^{1} u_{1}^{1} u_{1^{\prime}}^{1} \equiv & \left\{Q _ { + } ^ { - 2 } \left[\left(q^{-1} a_{+}+q b_{+}\right) q\left(1+q q_{+}\right) a_{+}+\left(a_{+}-b_{+}\right) z^{-1}\left(q^{-1}+q^{-1} z q_{+}\right) a_{+}\right.\right. \\
& \left.\left.+\left(q_{+} a_{+}+q_{-} b_{+}\right)\left(\varphi^{+}+\varphi^{-}\right) Q_{+}\right]+m_{+}\left(\varphi^{+}+\varphi^{-}-\mathfrak{s}\right)+1\right\} u_{1^{\prime}}^{1} .
\end{aligned}
$$

By Lemma 8.3, (iii.2), $u_{1^{\prime}}^{1} u_{1}^{1} u_{1}^{1} \equiv v^{2} a_{+}^{2} u_{1^{\prime}}^{1}$. Comparing the coefficients of $u_{1^{\prime}}^{1}$, (i) follows.

From $u_{2}^{1} u_{1^{\prime}}^{1} \equiv 0$ by Lemma 8.3, (i), and $u_{1}^{2} u_{1^{\prime}}^{1}=q u_{1^{\prime}}^{1} u_{1}^{2}$ we find $u_{2}^{1} u_{1}^{2} u_{1^{\prime}}^{1} \equiv 0$. Since $D_{2^{\prime}}^{2}=q^{3} z^{-1}$ for $N \geqq 4$ and $D_{2}^{2}=1$, for $N=3$, we have $\left(u_{ \pm}\right)_{2}^{2}=Q_{+}^{-1}\left(q^{\mp 1} u_{2}^{2} \pm q^{2} z^{-1} u_{2^{\prime}}^{2^{\prime}}+q_{ \pm} U \mp Q q z^{-1} u_{1^{\prime}}\right)$ for all $N \geqq 3$. Using the latter expression, we treat the relation $u_{2}^{1} u_{1}^{2} u_{1}^{1} \equiv 0$ first by Lemma 8.3, (viii), and then by Lemma 8.3, (ii), (iii.3), (iii.4). Equating finally the coefficients of $u_{1}^{1}$, we obtain (ii).

Now we insert the above two solutions for $\varphi^{ \pm}$and $\psi^{ \pm}$into equations (i) and (ii) of Lemma 8.5. Note that $\varphi_{+}^{+}+\varphi_{+}^{-}-\mathfrak{s}=Q Z$ and $\varphi_{-}^{+}+\varphi_{-}^{-}-\mathfrak{s}=-Q Z-2 \mathfrak{s}$. One computes that $\mu^{ \pm}=\mu_{+}^{ \pm}$in the first case, and that $\mu^{ \pm}=\mu_{ \pm}^{ \pm}$in the second case if $Q Z+2 \mathfrak{s} \neq 0$. If $Q Z+2 \mathfrak{s}=0$, the equations (i) and (ii) have no solution in the second case. Note that except for the last summand on the left-hand side all terms in (i) and (ii) have the same values for both solutions. Therefore, $\mu_{+}^{ \pm} Q Z=\mu_{-}^{ \pm}(-Q Z-2 \mathfrak{s})$ for $Q Z+2 \mathfrak{s} \neq 0$, so it suffices to compute $\mu_{+}^{ \pm}$from (i) and (ii).

By Lemma 8.2 and Lemma 1.1, we have thus proved that $\mathscr{R}_{12}=\mathscr{B}_{+}$and so $\mathscr{R}=\mathscr{R}_{+}$or that $\mathscr{R}_{12}=\mathscr{B}_{-}$and so $\mathscr{R}=\mathscr{R}_{-}$if $Q Z+2 \mathfrak{s} \neq 0$.

Next we show that the pair $\left(\Gamma_{ \pm}, d\right)$ has the properties asserted in the second paragraph of Theorem 6.2. As always, in case of $\left(\Gamma_{-}, d\right)$ we assume that $Q Z+2 \mathfrak{s} \neq 0$. It has been already noted in Sect. 6 that in this case the linear mappings $X_{+}$and $X_{-}$are invertible. Hence $\left\{\omega_{i j}:= \pm Q\left({ }_{1}^{1} X_{ \pm}\right)_{i j}^{k m} \eta_{k m}\right.$ : $i, j=1, \ldots, N\}$ is a basis of the vector space $\left(\Gamma_{ \pm}\right)_{\text {inv. Using }}$ (6.3), the formula $\left(\hat{R}^{-1}\right)_{y x}^{p a}=\Phi(\hat{R})_{y x}^{p a}=C_{r}^{y} \hat{R}_{x b}^{r p} B_{a}^{b}$ and finally Lemma 7.4, (v), we obtain 
for $\left(\Gamma_{+}, d\right)$

$$
\begin{aligned}
d u_{j}^{i} & =D_{s}^{r}\left(\eta_{r s} u_{j}^{i}-u_{j}^{i} \eta_{r s}\right) \\
& =D_{s}^{r} u_{p}^{i}\left({ }^{+} l_{k}^{r}\right)\left(u_{x}^{p}\right) \kappa\left({ }^{-} l_{s}^{m}\right)\left(u_{j}^{x}\right) \eta_{k m}-D_{s}^{r} u_{j}^{i} \eta_{r s} \\
& =u_{p}^{i} D_{s}^{r} \hat{R}_{x k}^{r p} \hat{R}_{s j}^{x m} \eta_{k m}-u_{p}^{i} D_{m}^{k} \delta_{p j} \eta_{k m} \\
& =u_{p}^{i}\left(B_{s}^{y}\left(\hat{R}^{-1}\right)_{y x}^{p a} C_{k}^{a} \hat{R}_{s j}^{x m}-D_{m}^{k} \delta_{p j}\right) \eta_{k m} \\
& =u_{p}^{i}\left({ }_{1}^{1}\left(\operatorname{tr}_{12} \hat{R}_{12}^{-1} \hat{R}_{23}\right)-{ }_{1}^{1} K\right)_{p j}^{k m} \eta_{k m} \\
& =u_{p}^{i}\left(Q_{1}^{1} X_{+}\right)_{i j}^{k m} \eta_{k m}=u_{p}^{i} \omega_{p j} .
\end{aligned}
$$

In case of $\left(\Gamma_{-}, d\right)$ we have to replace ${ }^{+} l_{k}^{r}\left(u_{x}^{p}\right)$ by $l_{k}^{r}\left(u_{x}^{p}\right)=-\hat{R}_{x k}^{r p}$ and the above reasoning gives $d u_{j}^{i}=u_{p}^{i}\left(-Q_{1}^{1} X_{-}\right)_{i j}^{k m} \eta_{k m}=u_{p}^{i} \omega_{p j}$, so that $P_{\mathrm{inv}}\left(d u_{j}^{i}\right)=\omega_{i j}$ in both cases. Further, by the definitions of $\omega$ and $\eta$ and by Lemma 7.4, (vii), we have $\omega=D_{j}^{i} \omega_{i j}=Q D_{j}^{i}\left({ }_{1}^{1} X_{+}\right)_{i j}^{k m} \eta_{k m}=Q D_{r}^{k}\left(\operatorname{tr}_{12} X_{+}\right)_{r}^{m} \eta_{k m}=Q Z D_{m}^{k} \eta_{k m}=Q Z \eta$ and hence $d a=(Q Z)^{-1}(\omega a-a \omega), \quad a \in \mathscr{A}, \quad$ for the calculus $\left(\Gamma_{+}, d\right)$. Similarly, $d a=(Q Z+2 \mathfrak{s})^{-1}(\omega a-a \omega), a \in \mathscr{A}$, for $\left(\Gamma_{-}, d\right)$.

The rest of the proofs of Theorems 6.1 and 6.2 is similar to the case of $S L_{q}(N)$. We only carry out the proof that the right ideal $\mathscr{R}_{\tau}$ is associated with the bicovariant calculus $\left(\Gamma_{\tau}, d\right)$. From $\left(f_{ \pm}\right)_{j}^{i}-\varphi_{\tau}^{ \pm}\left(u_{+}\right)_{j}^{i} \in \mathscr{R}_{\tau}$ and the definitions of $F_{ \pm}$and $\mathfrak{F}_{ \pm}$we compute that $F_{ \pm}\left(u_{j}^{i} u_{s}^{\mathrm{r}}\right)-\varphi_{\tau}^{ \pm}\left({ }_{1}^{2} \mathfrak{F}_{ \pm}\right)_{i j s}^{\tau x y} u_{y}^{x} \in \mathscr{R}_{\tau}$. Similarly, $G_{ \pm}\left(u_{j}^{i} u_{s}^{\tau}\right)-\psi_{\tau}^{ \pm}\left({ }_{1}^{2} \mathfrak{G}_{ \pm}\right)_{i j s}^{\tau x y} u_{y}^{x} \in \mathscr{R}_{\tau}$ and $H_{ \pm}\left(u_{j}^{i} u_{s}^{\tau}\right)-\mu_{\tau}^{ \pm}\left({ }_{1}^{2} \mathfrak{H}_{ \pm}\right)_{i j s}^{\tau x y} u_{y}^{x} \in \mathscr{R}_{\tau}$. Therefore, by (8.9) and Lemma 7.5, (i), $\left(u_{j}^{i} u_{s}^{\tau}\right)^{\sim}-\left(\left({ }_{1}^{2} K_{12}+{ }_{1}^{2} T_{\tau}\right)_{i j s}^{\tau x y} u_{y}^{x}\right)^{\sim} \in \mathscr{R}_{\tau}$, so these elements are annihilated by the functionals $\chi_{k m}$ of the corresponding bicovariant calculus. But, by (2.10), each functional $\chi_{k m}$ of $\left(\Gamma_{\tau}, d\right)$ annihilates $u_{j}^{i} u_{s}^{\tau}-\delta_{i j} u_{s}^{\tau}-$ $\left(B_{2}^{t} T_{\tau} C_{1}^{t}\right)_{i j s}^{\tau x y} u_{y}^{x}=u_{j}^{i} u_{s}^{\tau}-\left({ }_{1}^{2} K_{12}+{ }_{1}^{2} T_{\tau}\right)_{i j s}^{\tau x y} u_{y}^{x}$. Since $\mathscr{R}_{\tau} \cap \tilde{\mathscr{A}}_{1}=\{0\}$, we conclude from the preceding that $\mathscr{R}_{\tau}$ corresponds to $\left(\Gamma_{\tau}, d\right)$.

\section{The Classical Limits}

In this section we briefly consider the case $q=1$ and the limits of the calculi $\left(\Gamma_{ \pm}, d\right)$ for $q \rightarrow 1$. Let $G$ denote the corresponding classical simple Lie group $B_{n}, C_{n}$ or $D_{n}$. We set $\sigma=1$ for $B_{n}, D_{n}$ and $\sigma=-1$ for $C_{n}$.

Let $\mathscr{R}_{ \pm}=\mathscr{B}_{ \pm} \cdot \mathscr{A}$ be the right ideal of ker $\varepsilon$ as defined in Sect. 6, where the constants $\alpha_{ \pm}, \beta_{ \pm}^{ \pm}, \gamma_{ \pm}, \varphi_{+}^{ \pm}, \varphi_{-}^{ \pm}, \psi_{+}^{ \pm}, \psi_{-}^{ \pm}, \mu_{+}^{ \pm}, \mu_{ \pm}^{ \pm}$are replaced by their limits for $q \rightarrow 1$. That is, we have $\psi_{+}^{+}=\frac{1}{2} \sigma(N+3-\sigma), \varphi_{+}^{-}=\frac{1}{2} \sigma(N-3-\sigma)$ and $\mu_{+}^{ \pm}=\sigma(N \pm 2)$. Let $T_{ \pm}$denote the limits of the linear mappings $T_{ \pm}$from Sect. 6 as $q \rightarrow 1$. From Lemma 7.5, (i), it follows that

$$
\begin{aligned}
T_{+}= & \hat{R}_{12} \hat{R}_{23}+2(N \sigma-2)^{-1}(N-\sigma)^{-1}\left(\sigma K_{23}-K_{23} \hat{R}_{12}\right) \\
& +(N \sigma-2)^{-1}\left[\hat{R}_{23} \hat{R}_{12}+\hat{R}_{12} K_{23} \hat{R}_{12}-I-K_{12} K_{23}\right. \\
& \left.+\sigma\left(\hat{R}_{12}+K_{12} \hat{R}_{23}-\hat{R}_{23}-\hat{R}_{12} K_{23}\right)\right]
\end{aligned}
$$

and

$$
T_{-}=-T_{+}-2 K_{12}+2 \sigma N^{-1} K_{23} K_{12}+2\left(N^{2}-\sigma N\right)^{-1}\left(\sigma K_{23} \hat{R}_{12}-K_{23}\right) .
$$


As in Sect. 5, we obtain a bicovariant differential calculus $\left(\Gamma_{ \pm}, d\right)$ over $\mathscr{A}$ for $q=1$ if we replace the transformation $T_{ \pm}$in Theorem 6.2 by their limit as $q \rightarrow 1$. The same reasoning as in Sect. 5 shows that the right ideal $\mathscr{R}_{ \pm}$is associated with the bicovariant calculus $\left(\Gamma_{ \pm}, d\right)$. Lemmas 8.1 and 8.2 remain valid also for $q=1$. Arguing similarly to the proof of Lemma 8.2 we derive the decomposition of $\operatorname{ad}\left[\left((\operatorname{ker} \varepsilon)^{2}\right)_{12}\right.$ into irreducible components. In fact, we obtain that im $S_{ \pm}, \operatorname{im} H_{ \pm}, \operatorname{im}{ }_{1}^{1} P_{0} \subseteq(\operatorname{ker} \varepsilon)^{2}$ for $B_{n}, C_{n}$ and $D_{n}, \operatorname{im}{ }_{1}^{1} P_{+}, \operatorname{im} F_{ \pm}$, $\mathscr{G}_{ \pm} \subseteq(\operatorname{ker} \varepsilon)^{2}$ for $\bar{B}_{n}, D_{n}$ and $\operatorname{im}_{1} P_{-}, \operatorname{im} G_{ \pm}, \mathscr{F}_{ \pm} \subseteq(\operatorname{ker} \varepsilon)^{2}$ for $C_{n}$. From this we conclude easily that $(\operatorname{ker} \varepsilon)^{2} \nsubseteq \mathscr{R}_{-}$and that $(\operatorname{ker} \varepsilon)^{2} \subseteq \mathscr{R}_{+}$for all three series $B_{n}, C_{n}$ and $D_{n}$. Therefore, in the limit $q \rightarrow 1$ only the bicovariant calculus $\left(\Gamma_{+}, d\right)$ contains the classical differential calculus on the Lie group $G$ as a quotient.

\section{References}

[BR] Barut, A.O., Rạczka, R.: Theory of group representations and applications. PWN, Warsaw, 1977

[B] Bernard, D.: Quantum Lie algebras and differential calculus on quantum groups. Progr. Theor. Phys. Suppl. 102, 49-66 (1990)

[BW] Birman, J., Wenzl, H.: Braids, link polynomials and a new algebra. Trans. Am. Math. Soc. 313, 249-273 (1989)

[BM] Brzeziński, T., Majid, S.: A class of bicovariant differential calculi on Hopf algebras. Lett. Math. Phys. 26, 67-78 (1992)

[CSWW] Carow-Watamura, U., Schlieker, M., Watamura, S., Weich, W.: Bicovariant differential calculus on quantum groups $S U_{q}(N)$ and $S O_{q}(N)$. Commun. Math. Phys. 142, 605-641 (1991)

[C] Connes, A.: Non-commutative differential geometry. Publ. Math. IHES 62, 44-144 (1986)

[D] Drinfeld, V.G.: Quantum groups. In: Proceedings ICM 1986, Providence, RI: Am. Math. Soc., 1987, pp. 798-820

[FRT] Faddeev, L.D., Reshetikhin, N.Yu., Takhtajan, L.A.: Quantization of Lie groups and Lie algebras, Leningrad Math. J. 1, 193-225 (1990)

[H] Hammermesh, M.: Group theory and its application to physical problems. Reading, MA: Addison-Wesley, 1992

[J] Jurčo, B.: Differential calculus on quantized simple Lie groups. Lett. Math. Phys. 22, 177-186 (1991)

[L] Lusztig, G.: Quantum deformations of certain simple modules over enveloping algebras. Adv. Math. 70, 237-249 (1988)

[M] Manin, Yu.I.: Quantum groups and non-commutative geometry. Publications du C. R. M. 1561, Univ. of Montreal, 1988

[MH] Müller-Hoissen, F.: Differential calculi on the quantum group $G L_{p, q}(2)$. J. Phys. A. Math. Gen. 25, 1703-1734 (1992)

[PW] Parshall, B., Wang, I.: Quantum linear groups. Memoirs Am. Math. Soc. 439, Providence, RI, 1991

[Re] Reshetikhin, N.Yu.: Quantized universal enveloping algebras, the Yang-Baxter equation and invariants of links I. Preprint LOMI E-4-87, Leningrad, 1988

[R1] Rosso, M.: Algèbres enveloppantes quantifiées, groupes quantiques compacts de matrices et calcul différentiel non commutatif. Duke Math. J. 61, 11-40 (1990)

[R2] Rosso, M.: Finite dimensional representations of the quantum analog of the enveloping algebra of a complex simple Lie algebra. Commun. Math. Phys. 117, 581-593 (1988)

[SWZ] Schirrmacher, A., Wess, J., Zumino, B.: The two-parameter deformation of $G L(2)$, its differential calculus and Lie algebra. Z. Phys. C 49, 317-324 (1991)

[SS] Schmüdgen, K., Schüler, A.: Covariant differential calculi on quantum spaces and on quantum groups. C.R. Acad. Sci. Paris 316, 1155-1160 (1993). 
[St] Stachura, P.: Bicovariant differential calculi on $S_{\mu} U(2)$. Lett. Math. Phys. 25, 175-188 (1992)

[Su] Sudberry, A.: Canonical differential calculus on quantum linear groups and supergroups. Phys. Lett. B 284, 61-65 (1992)

[WZ] Wess, J., Zumino, B.: Covariant differential calculus on the quantum hyperplane. Nuclear Phys. B (Proc. Suppl.) 18, 302-312 (1990)

[Wo1] Woronowicz, S.L.: Twisted $S U$ (2) group. An example of noncommutative differential calculus. Publ. RIMS Kyoto Univ. 23, 117-181 (1987)

[Wo2] Woronowicz, S.L.: Differential calculus on compact matrix pseudogroups (quantum groups). Commun. Math. Phys. 122, 125-170 (1989)

Communicated by M. Jimbo 